\title{
miR-200 deficiency promotes lung cancer metastasis by activating cancer- associated fibroblasts
}

\begin{abstract}
Authors: Bin Xue ${ }^{1}$, Chen-Hua Chuang ${ }^{2}$, Haydn M. Prosser ${ }^{3,4}$, Cesar Seigi Fuziwara1, Claudia Chan $^{1}$, Neil Sahasrabudhe ${ }^{1}$, Maximilian Kühn ${ }^{1}$, Yalei Wu ${ }^{5}$, Jingqi Chen ${ }^{1}$, Anne Biton ${ }^{1,6}$, Caifu Chen $^{5}$, John Erby Wilkinson ${ }^{7}$, Michael T. McManus ${ }^{8}$, Allan Bradley ${ }^{3,4}$, Monte M Winslow ${ }^{2}$, $\mathrm{Bo} \mathrm{Su}^{9 \#}$, and Lin $\mathrm{He}^{1 \#}$
\end{abstract}

\section{Affiliations:}

${ }^{1}$ Division of Cellular and Developmental Biology, MCB department, University of California at Berkeley, Berkeley, CA 94705, USA.

${ }^{2}$ Department of Genetics, Stanford University School of Medicine, Stanford, CA 94305, USA

${ }^{3}$ The Wellcome Trust Sanger Institute, Wellcome Trust Genome Campus, Hinxton, Cambridge, CB10 1SA, UK

${ }^{4}$ Cambridge Institute of Therapeutic Immunology \& Infectious Disease. Department of Medicine, University of Cambridge, CB2 OAW, UK

5 Thermo Fisher Scientific, 180 Oyster Point Boulevard, South San Francisco, CA 94080, USA

${ }^{6}$ Bioinformatics and Biostatistics, Department of Computational Biology, USR 3756 CNRS, Institut Pasteur, Paris, France

7 Department of Pathology, University of Michigan Medical School, Ann Arbor, MI, 48109, USA

${ }^{8}$ Department of Microbiology and Immunology, University of California San Francisco Diabetes Center, WM Keck Center for Noncoding RNAs, University of California, San Francisco, San Francisco, CA 94143, USA

${ }^{9}$ Central Laboratory, Shanghai Pulmonary Hospital, Tongji University School of Medicine, Shanghai, 200433, China

\# Correspondence to: su_bo_s@hotmail.com and Ihe@berkeley.edu 


\begin{abstract}
Lung adenocarcinoma, the most prevalent lung cancer subtype, is characterized by its high propensity to metastasize. Despite the importance of metastasis in lung cancer mortality, its underlying cellular and molecular mechanisms remain largely elusive. Here, we identified miR200 miRNAs as potent suppressors for lung adenocarcinoma metastasis. miR-200 expression is specifically repressed in mouse metastatic lung adenocarcinomas, and miR-200 decrease strongly correlates with poor patient survival. Consistently, deletion of mir-200c/141 in the Kras $^{\text {LSL-G12D/+; }}$ Trp53 flox/flox lung adenocarcinoma mouse model significantly promoted metastasis, generating a desmoplastic tumor stroma highly reminiscent of metastatic human lung cancer. miR-200 deficiency in lung cancer cells promotes the proliferation and activation of adjacent cancer-associated fibroblasts (CAFs), which in turn elevates the metastatic potential of cancer cells. miR-200 regulates the functional interaction between cancer cells and CAFs, at least in part, by targeting Notch ligand Jagged1 and Jagged2 in cancer cells and inducing Notch activation in adjacent CAFs. Hence, the interaction between cancer cells and CAFs constitutes an essential mechanism to promote metastatic potential.
\end{abstract}




\section{Introduction}

Lung adenocarcinoma, the most prevalent lung cancer subtype, is characterized by its high propensity to metastasize. Metastatic lung adenocarcinoma cells escape primary tumors, disseminate via blood and lymphatic circulation, and ultimately colonize distant organs, particularly the liver, bone and brain ${ }^{1,2}$. This metastasis results in rapid disease progression and organ failure, ultimately accounting for the majority of patient mortality ${ }^{3}$. Despite its immense clinical significance, the cellular and molecular basis for lung adenocarcinoma metastasis remains largely unknown.

Both cancer cell intrinsic mechanisms and tumor-stroma interactions regulate metastatic progression ${ }^{4}$. Previous studies have mostly focused on intrinsic mechanisms that promote metastasis, involving transcription factors and signaling molecules that increase chromosome instability and accessibility, promote developmental plasticity, alter the cancer secretome, or enhance epithelial-mesenchymal transition (EMT) $)^{5-8}$. However, emerging evidence has implicated interactions between cancer cells and the tumor microenvironment, as lung adenocarcinoma metastases are frequently characterized by desmoplasia and substantial remodeling of the tumor microenvironment ${ }^{9,10}$. One major obstacle to study lung adenocarcinoma metastasis is the limited number of faithful animal models that recapitulates the entire metastatic processes in vivo. Widely used transplantation models largely depend on cancer cell lines, often bypassing important in vivo processes to achieve metastatic growth ${ }^{11}$, while popular genetically engineered mouse models (GEMMs) of lung adenocarcinoma often require a relatively long latency to develop metastasis ${ }^{12,13}$.

One of the most widely used models for metastatic lung adenocarcinoma is the Kras ${ }^{L S L-G 12 D /+}$; $\operatorname{Trp53}^{\text {floxflox }}(K P)$ model, in which inducible $K_{r a s}{ }^{G 12 D}$ expression and p53 deletion initiate the growth of lung adenocarcinomas that highly resemble human pathology ${ }^{14}$. While this model can eventually manifest metastatic tumors, its usefulness is hampered by a long latency period and incomplete penetrance ${ }^{12,13}$. Here, we identified miR-200 miRNAs as key suppressors for metastasis in KP lung adenocarcinomas. Engineered miR-200 deficiency promoted the rapid development of metastasis to lymph nodes and distant organs, faithfully recapitulating the desmoplastic tumor microenvironment characteristic of metastatic human disease. miR-200 deficiency in neoplastic cells increases the expression of Notch ligand Jag1 and Jag2.

Enhanced Notch signaling in neighboring stromal fibroblasts induced their proliferation and their remodeling into cancer-associated fibroblasts (CAFs), which in turn promoted the development 
of metastases. Our findings uncover key miRNAs that normally suppress metastasis by restricting CAF activation, and highlights the importance of tumor microenvironment remodeling during lung adenocarcinoma metastasis. 


\section{Results}

\section{Downregulation of $m i R-200$ miRNAs in a mouse model for metastatic lung cancer}

To compare the miRNA expression profiles between primary and metastatic lung

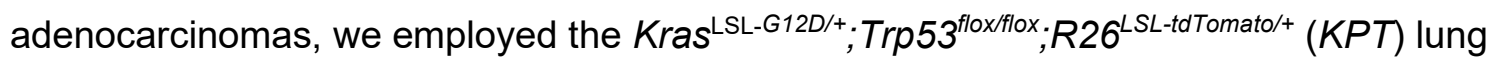
adenocarcinoma mouse model, in which inducible Kras activation and p53 loss in lung epithelial cells yield malignant lung adenocarcinomas with a long latency for metastasis ${ }^{12,13}$. Spontaneous metastasis emerged in lymph nodes, peritoneum, liver, adrenal gland, and soft tissue 5 to 9 months after tumor initiation induced by lenti-Cre administration ${ }^{13,15}$. KPT lung cancer cells were marked with the tdTomato Cre-reporter, which enabled purification of cancer cells from primary tumors and metastases (Fig. 1A). Among 641 miRNAs analyzed, 20 highly-expressed miRNAs were differentially expressed between non-metastatic primary tumors and metastases (Fig. 1B, Sup Fig. S1A, Supplementary Table S1). Strikingly, nearly all miR-200 miRNAs were repressed in metastases (Sup Fig. S1A). In particular, miR-141 and miR-200c were among the most downregulated miRNAs in metastatic KPT lung tumors (Fig. 1B, Sup Fig. S1A).

The miR-200 family contains five evolutionarily conserved miRNAs located in two distinct genomic loci, mir-200b/200a/429 on chromosome 4 and mir-200c/141 on chromosome 6 . They are classified into two subfamilies that differ by one nucleotide in their seed sequences (Fig. 1C). miR-200 miRNAs were expressed in both early and late stage primary lung adenocarcinomas, exhibiting only a slight downregulation during tumor progression (Fig. 1D). However, miR-200 expression was strongly repressed specifically in metastatic lung adenocarcinomas in KPT mice (Fig. 1D). Consistently, in the 432 lung adenocarcinomas analyzed by The Cancer Genome Atlas (TCGA) project, a collective, low miR-200 expression was correlated with poor overall patient survival ( ${ }^{* * *} P=0.0018$, Fig. 1E). Hence, downregulation of $m i R-200$ is associated with tumor metastasis in lung adenocarcinomas.

\section{miR-200 deficiency promotes the development of lung cancer metastases}

We next investigated the functional role of miR-200 miRNAs in lung cancer metastasis in the

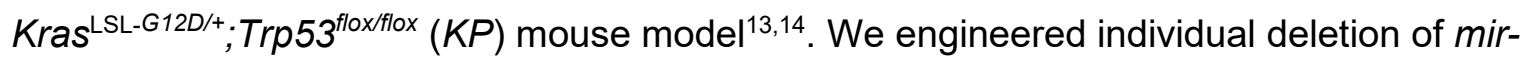
$200 c / 141$ or mir-200b/200a/429 in mice ${ }^{16}$ (Sup Fig. S1B). mir-200c/141/- mice were phenotypically normal, with no obvious developmental defects. However, deficiency of mir200b/200a/429 impaired fertility ${ }^{17}$, while mir-200c/141 ${ }^{-/} ;$mir-200b/200a/429-/- mice are neonatal 
lethal (data not shown). While further deletion of mir-200c/141 in KP mice (KP;mir-200c/141/-, designated as the KP200c) exhibit only partial miR-200 deficiency, they nonetheless provide essential insights into the role of miR-200 in lung cancer metastases.

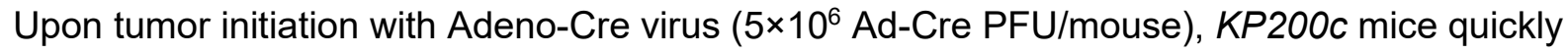
developed highly malignant lung adenocarcinomas. Advanced lesions with severe pleomorphic nuclei were present as early as one month after tumor initiation (Sup Fig. S1C). At the same time point, KP mice only had adenomas with mild nuclei atypia (Sup Fig. S1C). In line with these observations, KP200c mice exhibited a significant decrease in overall survival compared to $K P$ mice (KP200c median survival 90 days, KP median survival 111 days, ${ }^{* * *} P=0.0003$, log rank test, Fig. 1F, Supplementary Table S2).

At 12 weeks after tumor initiation, KP200c mice developed significantly larger and more aggressive lung adenocarcinomas, with a tumor burden 5 times as high as that of $K P$ mice (Fig. $1 G, 11)$. Nearly all KP200c lung tumors were grade 2 or above, with more than $65 \%$ between grade 3 to grade 5 , while $25 \%$ of $K P$ tumors were grade 1 and only $27 \%$ were grade 3 or above (Fig. 1I). High grade KP200c tumors were characterized by their large size, solid histological pattern, large and pleomorphic nuclei, and high degree of desmoplastic stroma surrounding the cancer cells (Fig. 1H).

miR-200c/141 deficiency not only accelerated tumor growth and progression, but also significantly increased the frequency of tumor metastasis. More than $60 \%$ of KP200c mice (20 out of 32 mice) developed lymph node (LN) metastases within 120 days, mostly occurring in mediastinal and thoracic lymph nodes (Fig. 1G, Fig 1J, Supplementary Table S2). Within the same time frame, only 1 in $26 K P$ mice developed LN metastasis (Fig $1 \mathrm{~J})$. When a lower dose of Cre-virus was used to initiate tumors in KP200c and KP mice $\left(1 \times 10^{5}\right.$ Lenti-Cre PFU/mouse), $50 \%$ of KP200c mice developed full-blown distant metastases to liver, pleura, pericardium, and adrenal gland by 150 days after tumor initiation (Fig. 1J, Fig. 1K, Sup Fig. S1E, Supplementary Table S2). Under the same experimental conditions, KP mice exhibited much lower primary tumor burden with no distant metastasis (Fig. 1J, Supplementary Table S2). Interestingly, all KP200c metastases exhibited a complete silencing of mir-200b/200b/429 (Sup Fig. S1D), suggesting a strong selective pressure for a complete miR-200 loss in metastatic Kras-driven, p53-null lung adenocarcinomas. Hence, KP200c mice provide a powerful new experimental system to probe the underlying mechanisms of adenocarcinoma metastasis. 


\section{miR-200 deficiency induces the expansion of CAFs in metastatic KP200c tumors}

Metastases in KP200c mice were accompanied by an increase in MAPK signaling and a loss of cell differentiation (Fig. 2A). Compared to KP primary tumors, phosphorylated Erk1/2 level was significantly increased in KP200c primary tumors ( $\left.{ }^{*} P=0.0263\right)$, and further elevated in $K P 200 c$ LN and distant metastases $\left({ }^{* *} P<0.0001\right)$ (Fig. $\left.2 A\right)$, indicating a synergic effect of $K r a s^{G 12 D}$ induction and mir-200c/141 deficiency on MAPK activation during metastasis progression. In addition, $64 \%$ of lymph node and distant metastases from KP200c mice were negative for both surfactant protein C (SPC), a marker for type 2 alveolar epithelial cells (AT2), and club cell antigen 10 (CC10), a marker for bronchiolar club cells (Fig. 2B). KP200c primary tumors, in comparison, were more heterogeneous in the expression of SPC and CC10, with only $28 \%$ of tumors being $\mathrm{SPC}{ }^{\text {low }} \mathrm{CC} 10^{\text {low }}$, and the remaining exhibiting varying degrees of SPC and $\mathrm{CC} 10$ expression (Fig. 2B, Sup Fig. S2A). Nearly all KP200c metastases lost expression of Nkx2.1 (Fig. 2C), a lung lineage transcription factor that controls lung tumor differentiation and limits metastatic potential ${ }^{6}$. KP200c primary tumors, in comparison, mostly expressed Nkx2.1 with downregulated expression in some high-grade regions (Fig. 2C). Our findings suggest that KP200c metastases could be derived from poorly differentiated cancer cells with highly elevated MAPK signaling.

A prominent cellular hallmark of metastatic KP200c tumors was the abundance of collagen-rich, stromal desmoplasia within the metastatic tumor microenvironment. In comparison, $K P$ tumors only exhibited widespread desmoplasia in liver metastases, but not in the lymph node metastases nor in primary tumors (Sup Fig. S2B, S2C, S2D). A major source of extracellular matrix in the tumor microenvironment came from CAFs, a heterogeneous population of fibroblasts that promote cancer growth, survival, and invasiveness by secreting cytokines and growth factors (interleukins, HGF, CXCLs, TGF- $\beta$ etc. ${ }^{18-24}$, as well as collagen to increase tissue stiffness ${ }^{25}$. In KP200c late-stage primary tumors, lymph node metastases, and distant metastases, we observed a marked increase of $\alpha$-smooth muscle actin-expressing CAFs ( $\alpha$ SMA+ CAFs) (Fig. 2D), which grew into multiple layers and intertwined with clusters of KP200c cancer cells (Fig. 2D). This multilayered, $\alpha-S M A+$, stromal structure was largely absent in $K P$ primary tumors, including high-grade tumors (Fig. 2D). Unlike a-SMA+ CAFs, FSP-1-expressing CAFs did not show a significant increase in KP200c tumors (Sup. Fig S2E). Importantly, the percentage of $\alpha$-SMA + CAFs in KP200c lymph node metastasis was proportional to the tumor size (Fig. 2E), with $70 \%$ of KP200c lymph node metastases containing at least $20 \%$ of $\alpha-S M A+$ CAFs (Fig. 2E). $\alpha$-SMA staining was absent from the epithelial compartment in both $K P$ and 
KP200c normal lung and early lesions (Fig. 2F). Our findings suggest that miR-200 deficiency in lung adenocarcinomas lead to the recruitment and expansion of $\alpha-S M A+C A F s$ in the tumor microenvironment, which could be a critical step for the development of metastatic lung adenocarcinomas.

In KP200c mice, both neoplastic cells and stromal cells were deficient for mir-200c/141, making it difficult to determine whether mir-200c/141 deficiency in cancer cells and/or stromal cells drove tumor growth and metastasis. To address the role of mir-200c/141 specifically in neoplastic cells, we generated KP mice with a mir-200c/141 conditional allele (Kras ${ }^{\mathrm{G12D/+}}$; p53 flox/flox; mir-200c/141 flox/-, designated as KP200cCKO mice $)^{26}$. This model allows the generation of mir-200c/141-deficient $K P$ cancer cells in an otherwise mir-200c/141-expressing tumor microenvironment (Sup Fig. S2F). Similar to KP200c mice, KP200cCKO mice exhibited an increase in metastasis frequency, as $80 \%$ of KP200cCKO mice developed lymph node or distant metastases by 120 days after tumor initiation (Fig. 2G, Supplementary Table S2, Sup Fig. S2G). Metastases in KP200cCKO mice resembled those of KP200c mice; both were characterized by strong desmoplasia with extensive infiltration of $\alpha-S M A+C A F s$ (Fig. $2 G$ ). Hence, miR-200 deficiency in lung cancer cells promotes local and distant metastases, while inducing the expansion of $\alpha-S M A+C A F s$ in the tumor microenvironment.

\section{Enrichment of SMA+ CAFs occurs in human metastatic lung adenocarcinomas}

Consistent with a-SMA+ CAF enrichment in metastatic KP200c mouse lung tumors, a-SMA+

CAFs were also enriched in metastatic human lung adenocarcinomas (Fig. $2 \mathrm{H}$ ). We compared SMA staining in paired primary lung adenocarcinomas and lymph node metastases from 8 patients, and we observed a significant increase of $\alpha-S M A+C A F s$ in lymph node metastases in 7 out of 8 patients (Fig. $2 \mathrm{H}$ ). Several patients exhibited at least a two-fold increase in the number of $\alpha-S M A+C A F s$ in lymph node metastases compared to their paired primary tumors (Fig. 2H). Two patients exhibited a similar enrichment of $\alpha-S M A+C A F s$ in liver metastases relative to their paired primary tumors (Fig. 2l).

Interestingly, we captured a continuum of CAF activation patterns in a stage IA human lung adenocarcinoma (Fig. 2J). In normal lung, the thin alveoli septa consists of a monolayer of $\alpha$ SMA+ pericytes embedded in the basement membrane of type 1 alveolar cells (AT1) (Fig. 2J, area a). When the lesion progressed to atypical adenomatous hyperplasia (AAH), the 
transformed epithelial cells grew into the alveolar space with the $\alpha-S M A+C A F s$ starting to expand within the alveolar septa (Fig. 2J, area b). In high-grade adenocarcinomas where the alveolar lumen is filled with cancer cells (Fig. 2J, area c), the $\alpha-S M A+C A F s$ formed multilayered structures intermingled with cancer cells. Hence, the expansion of the $\alpha-S M A+C A F s$ in the tumor microenvironment, as well as the extent of tumor-CAF interaction, both correlate with histological progression in human adenocarcinomas.

\section{Fibroblast co-culture promotes the metastatic features in KP200c tumor organoids} We next established a 3D tumor organoid system by culturing one KP and three KP200c lung cancer cell lines (Sup Fig. S3A) on top of a matrix containing $50 \%$ Matrigel $+0.5 \mathrm{mg} / \mathrm{ml}$ Collagen I to mimic a collagen-rich lung tumor microenvironment (Fig. 3A). When cultured alone, KP and KP200c cancer cells both formed well-polarized spherical tumor organoids with hollow lumina (Fig. 3B), with KP200c organoids slightly larger in size (Fig. 3B), but with neither exhibiting invasive features (Fig. 3B). Interestingly, when co-cultured with primary lung fibroblasts, the miR-200 deficient KP200c organoids, but not the miR-200 expressing-KP organoids, clustered to form large multi-acinar structures with cellular protrusions, indicative of invasive behavior (Fig. 3C, 3D). This clustering behavior substantially increased the size of KP200c tumor organoids in culture (Fig. 3B, Sup Fig. S3B). The extent of organoid clustering, along with the degree of cell proliferation and invasiveness in KP200c organoids, were proportional to the ratio of fibroblasts to cancer cells (Sup Fig. S3C). Consistent with these findings, re-expression of mir-200c/141 in KP200c cancer cells co-cultured with lung fibroblasts reverted this invasive phenotype, restoring a spherical organoid morphology (Sup Fig. S3D, S3F). Hence, miR-200 miRNAs act in lung cancer cells to restrict the metastatic phenotype, at least in part, by regulating the functional interaction between cancer cells and fibroblasts.

\section{miR-200 deficiency in tumors promotes the proliferation and activation of CAFs.}

The interaction between KP200c cancer cells and stromal fibroblasts induced invasive features in neoplastic cells, while promoting their proliferation in 3D organoid culture (Fig 3E), but not in 2D culture (Sup Fig S3E). Increased fibroblast proliferation was only observed in the vicinity of KP200c tumor organoids (Fig. 3B), as tumor-associated fibroblasts exhibited a 3.8-fold increase in cell density compared to non-tumor-associated fibroblasts (Fig. 3F). No proliferation 
difference was observed between $K P$ tumor-associated and non-tumor-associated fibroblasts

(Fig. 3F). KP200c tumor-associated fibroblasts exhibited elongated morphology, induction of $\alpha$ SMA, and infiltration into tumor organoids to form direct cell-cell contact with cancer cells (Fig. $3 G)$. Consistently, highly proliferative $\alpha-S M A+C A F s$ with an elongated morphology were prevalent in KP200c high-grade primary tumors, lymph node, and distant metastases, but not in low-grade primary tumors (Fig. 3H, Sup Fig S3G).

miR-200 deficiency in lung cancer cells not only promotes the proliferation of surrounding lung fibroblasts, but also regulates their activity. A major function of CAFs is to secrete cytokines, chemokines, and growth factors to promote tumor growth, survival, and invasion ${ }^{18}$. We isolated tdTomato+ lung fibroblasts that were cultured alone or co-cultured with KP200c tumor organoids, and compared their expression of CAF-derived secreted factors. Fibroblasts cocultured with KP200c tumor organoids exhibited a strong induction of hepatocyte growth factor (HGF), TGF- $\beta$, fibroblast growth factor-1 (Fgf1) and Ccl5 (Fig. 3I), all of which have been reported to contribute to metastatic potential in different cancer models including lung adenocarcinoma ${ }^{27-33}$. Interestingly, mir-200c/141 re-expression in KP200c tumor organoids reduced the induction of these growth factors and cytokines in co-cultured fibroblasts (Fig. 3I), suggesting that miR-200 miRNAs in cancer cells regulate CAF gene expression via a cell nonautonomous mechanism. Moreover, treating KP200c cancer cells with HGF, TGF- $\beta$, or a combination of both is sufficient to induce the invasive behavior, resulting in enlarged invasive tumor organoid clusters (Sup Fig S3H).

\section{Interplay between KP200c cancer cells and fibroblasts promotes metastatic potential in vivo.}

Our findings implicate a complex interplay between cancer cells and CAFs regulated by miR200 miRNAs. To investigate the importance of cancer-fibroblast interactions in promoting metastases, we established an orthotropic allograft lung tumor model, in which we intratracheally transplanted lung cancer cells with or without primary lung fibroblasts into immune deficient recipient mice (Fig. 3J). Among two KP and three KP200c lung cancer lines tested, KP lines rarely grafted to form tumors, yet all KP200c lines successfully developed allograft lung tumors by 7 weeks (Sup Fig. S3I). When KP200c cancer cells were injected with co-cultured, tdTomato-labeled, primary lung fibroblasts, tumor growth was significantly accelerated with an increased tumor burden (Fig. 3K, Sup Fig. S3J). More significantly, within a 
short latency of 40 days, all mice exhibited early onset distant metastases affecting the pleural membrane in rib cage, pericardium, adrenal gland, kidney, and subcutaneous soft tissue (Fig. $3 \mathrm{~K}$, Sup Fig. S3K). In comparison, mice injected with KP200c cancer cells alone, while developing lung tumors, rarely developed metastases at this time point (Fig. 3K, Sup Fig. S3K). These allograft KP200c tumors likely gain metastatic potential eventually, as they promoted the proliferation and activation of endogenous stromal fibroblasts in recipient mice. Altogether, our data demonstrate that boosting cancer-fibroblast interactions in KP200c cancer cells significantly accelerated tumor outgrowth and metastases in vivo.

Surprisingly, when KP200c cancer cells were co-transplanted with primary lung fibroblasts into recipient mice, tdTomato+ fibroblasts could be detected within the disseminating cancer cell clusters in the pleural cavity and in distant metastases (Fig. 3L). Similarly, in KP200c mice, we observed cancer cell clusters that contained a-SMA+ CAFs inside blood/lymphatic vessels in the lung (Sup Fig S3L). Consistently, in a human patient with minimally invasive lung adenocarcinomas and lymph node micro-metastases, we detected clusters of cells containing $\alpha$ SMA+ CAFs adjacent to CK19+ cancer cells in blood (Sup Fig. S3M), and observed lymph node micro-metastases encased by a single layer of $\alpha-S M A+C A F s$ in the vicinity of lymphatic vessels (Sup. Fig S3N). These findings suggest that $\alpha-S M A+C A F s$ can adhere to metastatic cancer cells during dissemination and extravasation, and prolonged tumor-CAF interaction could play a role throughout different stages of tumor metastasis.

\section{miR-200 miRNAs repress Jag1/Jag2 and inhibit Notch signaling in CAFs to repress metastasis}

Cancer cells can convert lung fibroblasts into tumor-promoting CAFs via paracrine signaling or direct cell-cell interactions. Interestingly, KP200c cancer cells induced lung fibroblast proliferation and activation in a tumor organoid co-culture system that permitted cell-cell contact, but not in trans-well assays that separated cancer cells and fibroblasts in different compartments to prohibit cell-cell contacts (Fig. 4A). Hence, miR-200 miRNAs likely regulate pathways for direct cell-cell interactions between cancer cells and CAFs. 
Many studies suggest that a key function of miR-200 miRNAs is to suppress EMT by targeting Zeb1 and Zeb2 ${ }^{34-38}$. However, primary and metastatic KP200c tumors remained largely intact in epithelial morphology (Sup Fig. S4A), and failed to exhibit miR-200-dependent regulation of Zeb1, Zeb2, Vimentin, and E-cadherin (Sup Fig. S4B, S4C). Furthermore, Zeb1 and Zeb2 knockdown in KP200c lung cancer cells did not alter the invasive morphology of KP200c tumor organoids (Sup Fig S4D, S4E). We cannot exclude the possibility that transient EMT still occurs during lung cancer metastasis in vivo, since miR-200-deficient lung cancer cells are more susceptible to TGF- $\beta$-induced EMT ${ }^{34}$. Yet our data strongly suggest that the miR-200-EMT pathway is not required for cancer-CAF interactions which play an essential role in promoting metastasis.

Gene ontology analysis on predicted miR-200 targets revealed multiple candidates involved in cell-cell communication, including Jagged1 (Jag1), Jagged2 (Jag2), Postn, Gjc1, and Pcdh9, as predicted by RNA22 and TargetScan algorithms ${ }^{39,40}$. Notch ligands Jag1 and Jag2 emerged as strong candidate $m i R-200$ targets, each containing a predicted $m i R-141$ or $m i R-200 \mathrm{c}$ binding site, respectively (Fig. 4B). Furthermore, their mRNA expression was elevated in miR-200deficient metastatic cancer cells where lost the expression of both mir-200 loci (Fig. 4C, Sup Fig. S1D), but reduced upon miR-200 re-expression (Fig. 4D). Hence, the di-cistronic miRNA cluster mir-200c/141 acts coordinately to downregulate both Jag1 and Jag2 expression in cancer cells.

Notch signaling operates in diverse developmental and pathological processes. Notch ligands, including Jag1, Jag2, DII1, and DII4, bind to membrane-bound Notch receptors in neighboring cells, inducing a cascade of proteolytic events that ultimately release the Notch intracellular domain (NICD) which translocates into the nucleus ${ }^{41-43}$. In the nucleus, NICD interacts with DNA binding protein RBPJ and co-activator MAML to stimulate the transcription of Notch target genes, such as Hes, Hey, and Nrarp ${ }^{44,45}$. Notch expressing cells are often activated by cell surface ligands in adjacent cells, and Notch ligand producing cells are known to dampen their own Notch activity through cis-inhibition ${ }^{46}$. Consistently, miR-200 deficiency in KP200c cancer cells resulted in elevated expression of Notch target genes Hes1 and Nrarp in co-cultured fibroblasts, but a decrease in Notch target gene expression in KP200c cancer cells (Fig. 4E). This induction of Notch activity in co-cultured fibroblasts was reversed by re-expressing miR200c/141 in KP200c cancer cells (Fig. 4F). 
To investigate the sufficiency of elevated fibroblast Notch signaling to promote the metastatic potential of cancer cells, we manipulated Notch signaling in lung fibroblasts and examined their effects on co-cultured tumor organoids. Since Notch1 and Notch3 are the main Notch receptors expressed in lung fibroblasts (data not shown), we activated Notch signaling in lung fibroblasts by overexpressing Notch1 NICD (NICD1) or Notch3 NICD (NICD3), and co-cultured them with miR-200c/141-re-expressing KP200c tumor organoids. While miR-200 expression abolished metastatic features of KP200c tumor organoids when co-cultured with normal lung fibroblasts (Sup Fig. S3F), co-culture with NICD1 and NICD3 expressing fibroblasts reversed the effects of miR-200 re-expression in KP200c tumor organoids, promoting cancer cell clustering and invasive cellular features (Fig. 4G). In line with these findings, CAFs in metastatic KP200c tumors exhibited specific activated Notch1 nuclear staining (Fig. 4H, left), indicative of a strong Notch activation in these cells. Similar observations were made in two human lung adenocarcinoma samples, where activated Notch1 nuclear staining was evident in CAFs associated with metastases (Fig.4H, Sup Fig. S4G).

Consistent with Jag1 and Jag2 being the key miR-200 targets that mediate tumor-CAF interactions, knocking down Jag1 and Jag2 in KP200c cancer cells restored a spherical morphology in tumor organoids, abolished tumor clustering and invasive features, and reduced fibroblasts elongation and expansion (Fig. 4I). Hence, Jag1 and Jag2 derepression caused by miR-200 loss in cancer cells triggered Notch activation in adjacent CAFs, which promoted fibroblast proliferation and activation, ultimately enhancing the metastatic potential of cancer cells (Fig. 4J).

\section{Discussion}

miR-200 miRNAs act as potent suppressors of lung cancer metastasis, and complete miR-200 inactivation was observed in metastatic lung adenocarcinomas in both KP and KP200c models. The KP200c model is among the best for studying lung adenocarcinoma metastasis, owing to its short latency and faithful recapitulation of patient pathology. Metastatic KP200c lung adenocarcinomas faithfully recapitulate the desmoplastic stroma found in human metastatic tumors $^{9,47}$, characterized by a pro-metastatic niche filled with SMA+ CAFs. These SMA+CAFs, derived from normal lung fibroblasts, strongly foster the metastatic potential of cancer cells, at least in part, by secreting cytokines and growth factors (Fig. 3G, 3I). A recent study showed that human metastatic lung adenocarcinomas possess transcriptomes encompassing a 
developmental continuum ranging from stem cell-like cells to regenerative progenitors in lung epithelia ${ }^{48}$. It is likely that CAFs in the desmoplastic microenvironment not only promote tumor cell proliferation, but also create a pro-metastatic niche to induce developmental plasticity in cancer cells. This is consistent with the reports that Wnt-expressing fibroblasts establish a stem cell niche for AT2 cells in normal development and for cancer stem cells in lung adenocarcinoma ${ }^{49,50}$. Given the heterogeneity of the tumor microenvironment, CAF-dependent pro-metastatic niches are likely to require interactions between CAFs and other stromal cell types in lung adenocarcinomas.

In most studies, miR-200 miRNAs are characterized as key inhibitors of EMT by repressing Zeb1 and Zeb2 ${ }^{34-38}$. Yet EMT may not be essential for metastasis in KP200c lung adenocarcinomas, as overt EMT was not observed in metastatic KP200c tumors, and Zeb1 and Zeb2 knockdown failed to reverse invasiveness in tumor organoids (Sup Fig. S4A, S4D, S4E). Rather, miR-200 deficiency in KP200c cancer cells leads to increased Jag1 and Jag2, elevating Notch signaling in neighboring fibroblasts to promote their conversion into a-SMA+ CAFs, thereby establishing a pro-metastatic niche. This Notch activation in CAFs is strongly associated with metastatic lung adenocarcinomas in both mouse models and human patients (Fig. $4 \mathrm{H})$, highlighting the importance of the miR-200/Jag/Notch axis in orchestrating cancer-CAF interactions to promote metastasis in lung adenocarcinomas. Notch activation in lung fibroblasts also induces $\alpha-S M A$ expression and TGF- $\beta$ secretion during lung fibrosis ${ }^{51}$. Hence, Notch signaling regulates fibroblast dependent, cell-cell interactions in multiple lung pathological processes.

The effects of Notch signaling are complex in various tumor types. Notch activation in fibroblasts promotes CAF activation and tumor progression in lung, breast, and prostate cancer ${ }^{52,53}$, and the loss of Notch signaling in CAFs promotes an oncogenic tumor microenvironment in squamous cell carcinomas ${ }^{54,55}$. It is important to recognize that the unique cellular heterogeneity found within each tumor microenvironment could foster a complex crosstalk between cancer cells and stromal cells. In both mouse and human metastatic lung adenocarcinomas, miR-200 deficient lung cancer cells could also induce Notch signaling in other stromal cell types, which in turn promoted metastasis. Taken together, miR-200 miRNAs regulate Notch signaling, which mediate important cell-cell interactions between cancer cells and stromal cells to suppress lung cancer metastasis. 


\section{Acknowledgements:}

We thank H. Nolla, H. Kartoosh and A. Valeros from for FACS support; A.M. Li for establishing a collaboration with Dr. Bradley's lab; A. Holly, J.Y. Lee and F. Ives from Molecular Imaging Center at UC Berkeley and M. West and P. He from HTS facility at UC Berkeley for imaging assistance; Functional Genomics Laboratory at UC Berkeley for genomics services, C. Hung for experimental help and proofreading the manuscript, S. Chen for revision advice, G. Lee, A. Nazarenko, T. Colston and S. Yue for experimental help and mouse colony management; C. Fellmann for providing SGEP and SGEN vectors; M. Junttila, R. Molina and J. Long from Genentech for intranasal injection protocol. H. Ji and F. Li for Kras ${ }^{G 12 D /+;} L k b 1^{\text {flox/flox }}$ tumor samples. N. Arpaia, K.A. Kaiser and all He lab members, in particular N. Okada, M. Bennett, E. Ho and J. Schwedler for suggestions and discussions. L.H. is a Thomas and Stacey Siebel Distinguished Chair Professor, who is supported by a Howard Hughes Medical Institute (HHMI) Faculty Scholar award, a Bakar Fellow award, at UC Berkeley, and several grants from the National Institutes of Health (NIH, R01CA139067 and 1R21OD027053). M.M. W. was supported by NIH R01-CA175336 and R01-CA204620. C.H.C was funded by an American Lung Association Fellowship. B. S. was supported by Grant No.81572269, National Natural Science Foundation of China.

Author Contribution: B.X., B.S., M.M.W., and L.H. conceived, designed, and directed the study. B.X. performed most mouse experiments and generated the majority of the mouse data for this study; B.S. performed most experiments involving human samples, and has contributed his clinical expertise to this study; C.H.C and M.M.W. generated and isolated KP tumors and metastases for miRNA analysis. J.C. and A.B. performed survival analysis of human data from TCGA. J.E.W. performed pathology analysis on all the mice tumors. C.C., N.S., C.S.F. and M.K. performed mouse tumor histology, IHC staining and molecular cloning experiments. Y.W. and C.C. designed and provided miRNA TaqMan analysis. H.M.P and A.B. generated miR-200c/141 null mice. M.T.M. generated mir-200c/141/acz mice. B.X. and L.H. wrote the manuscript with input from all authors.

\section{Competing interests:}

Authors declare no competing interests. 


\section{Data and materials availability:}

All data is available in the main text or the supplementary materials. All data and reagents will be available to any researcher for purposes of reproducing or extending the analysis. 


\section{References:}

1. Budczies, J. et al. The landscape of metastatic progression patterns across major human cancers. Oncotarget 6, 570-83 (2015).

2. Obenauf, A. C. \& Massagué, J. Surviving at a Distance: Organ-Specific Metastasis. Trends in Cancer 1, 76-91 (2015).

3. Howlader N, Noone AM, Krapcho M, Miller D, Brest A, Yu M, Ruhl J, Tatalovich Z, Mariotto A, Lewis DR, Chen HS, Feuer EJ, C. K. (eds). SEER Cancer Statistics Review, 1975-2017. National Cancer Institute. Bethesda, MD (2020).

4. Quail, D. F. \& Joyce, J. A. Microenvironmental regulation of tumor progression and metastasis. Nat. Med. 19, 1423-1437 (2013).

5. Celià-Terrassa, T. \& Kang, Y. Distinctive properties of metastasis-initiating cells. Genes Dev. 30, 892-908 (2016).

6. Winslow, M. M. et al. Suppression of lung adenocarcinoma progression by Nkx2-1. Nature 473, 101-104 (2011).

7. Denny, S. K. et al. Nfib Promotes Metastasis through a Widespread Increase in Chromatin Accessibility. Cell 166, 328-342 (2016).

8. Sinkevicius, K. W. et al. Neurotrophin receptor TrkB promotes lung adenocarcinoma metastasis. Proc. Natl. Acad. Sci. 111, 10299-10304 (2014).

9. Bremnes, R. M. et al. The Role of Tumor Stroma in Cancer Progression and Prognosis: Emphasis on Carcinoma-Associated Fibroblasts and Non-small Cell Lung Cancer. J. Thorac. Oncol. 6, 209-217 (2011).

10. Altorki, N. K. et al. The lung microenvironment: an important regulator of tumour growth and metastasis. Nat. Rev. Cancer 19, 9-31 (2019).

11. Gómez-Cuadrado, L., Tracey, N., Ma, R., Qian, B. \& Brunton, V. G. Mouse models of metastasis: progress and prospects. Dis. Model. Mech. 10, 1061-1074 (2017).

12. Jackson, E. L. et al. The differential effects of mutant p53 alleles on advanced murine lung cancer. Cancer Res. 65, 10280-8 (2005).

13. DuPage, M., Dooley, A. L. \& Jacks, T. Conditional mouse lung cancer models using adenoviral or lentiviral delivery of Cre recombinase. Nat. Protoc. 4, 1064-72 (2009).

14. Jackson, E. L. et al. Analysis of lung tumor initiation and progression using conditional 
expression of oncogenic K-ras. Genes Dev. 15, 3243-8 (2001).

15. Caswell, D. R. et al. Obligate Progression Precedes Lung Adenocarcinoma Dissemination. Cancer Discov. 4, 781-9 (2014).

16. Prosser, H. M., Koike-Yusa, H., Cooper, J. D., Law, F. C. \& Bradley, A. A resource of vectors and ES cells for targeted deletion of microRNAs in mice. Nat. Biotechnol. 29, 840-5 (2011).

17. Hasuwa, H., Ueda, J., Ikawa, M. \& Okabe, M. MiR-200b and miR-429 Function in Mouse Ovulation and Are Essential for Female Fertility. Science 341, 71-73 (2013).

18. Gascard, P. \& Tlsty, T. D. Carcinoma-associated fibroblasts: orchestrating the composition of malignancy. Genes Dev. 30, 1002-1019 (2016).

19. Ahirwar, D. K. et al. Fibroblast-derived CXCL12 promotes breast cancer metastasis by facilitating tumor cell intravasation. Oncogene 37, 4428-4442 (2018).

20. Bhowmick, N. A., Neilson, E. G. \& Moses, H. L. Stromal fibroblasts in cancer initiation and progression. Nature 432, 332-337 (2004).

21. Frantz, C., Stewart, K. M. \& Weaver, V. M. The extracellular matrix at a glance. Journal of Cell Science 123, 4195-4200 (2010).

22. Kalluri, R. \& Zeisberg, M. Fibroblasts in cancer. Nature Reviews Cancer 6, 392-401 (2006).

23. Orimo, A. et al. Stromal fibroblasts present in invasive human breast carcinomas promote tumor growth and angiogenesis through elevated SDF-1/CXCL12 secretion. Cell 121, 335-348 (2005).

24. Pietras, K. \& Östman, A. Hallmarks of cancer: Interactions with the tumor stroma. Experimental Cell Research 316, 1324-1331 (2010).

25. Pankova, D. et al. Cancer-Associated Fibroblasts Induce a Collagen Cross-link Switch in Tumor Stroma. Mol. Cancer Res. 14, 287-95 (2016).

26. Park, C. Y. et al. A Resource for the Conditional Ablation of microRNAs in the Mouse. Cell Rep. 1, 385-391 (2012).

27. Ao, M. et al. Cross-talk between Paracrine-Acting Cytokine and Chemokine Pathways Promotes Malignancy in Benign Human Prostatic Epithelium. Cancer Res. 67, 42444253 (2007). 
28. Karnoub, A. E. et al. Mesenchymal stem cells within tumour stroma promote breast cancer metastasis. Nature 449, 557-563 (2007).

29. Kojima, Y. et al. Autocrine TGF- and stromal cell-derived factor-1 (SDF-1) signaling drives the evolution of tumor-promoting mammary stromal myofibroblasts. Proc. Natl. Acad. Sci. 107, 20009-20014 (2010).

30. Straussman, R. et al. Tumour micro-environment elicits innate resistance to RAF inhibitors through HGF secretion. Nature 487, 500-504 (2012).

31. Sun, Y. et al. Cancer-associated fibroblasts secrete FGF-1 to promote ovarian proliferation, migration, and invasion through the activation of FGF-1/FGFR4 signaling. Tumor Biol. 39, 1-10 (2017).

32. Yu, J.-R. et al. TGF- $\beta /$ Smad signaling through DOCK4 facilitates lung adenocarcinoma metastasis. Genes Dev. 29, 250-261 (2015).

33. Borczuk, A. C. et al. Lung adenocarcinoma invasion in TGFßRII-deficient cells is mediated by CCL5/RANTES. Oncogene 27, 557-564 (2008).

34. Gibbons, D. L. et al. Contextual extracellular cues promote tumor cell EMT and metastasis by regulating miR-200 family expression. Genes Dev. 23, 2140-2151 (2009).

35. Gregory, P. A. et al. An autocrine TGF- $\beta / Z E B / m i R-200$ signaling network regulates establishment and maintenance of epithelial-mesenchymal transition. Mol. Biol. Cell 22, 1686-1698 (2011).

36. Korpal, M., Lee, E. S., Hu, G. \& Kang, Y. The miR-200 family inhibits epithelialmesenchymal transition and cancer cell migration by direct targeting of E-cadherin transcriptional repressors ZEB1 and ZEB2. J. Biol. Chem. 283, 14910-4 (2008).

37. Park, S.-M., Gaur, A. B., Lengyel, E. \& Peter, M. E. The miR-200 family determines the epithelial phenotype of cancer cells by targeting the E-cadherin repressors ZEB1 and ZEB2. Genes Dev. 22, 894-907 (2008).

38. Schliekelman, M. J. et al. Targets of the tumor suppressor miR-200 in regulation of the epithelial-mesenchymal transition in cancer. Cancer Res. 71, 7670-82 (2011).

39. Miranda, K. C. et al. A Pattern-Based Method for the Identification of MicroRNA Binding Sites and Their Corresponding Heteroduplexes. Cell 126, 1203-1217 (2006).

40. Agarwal, V., Bell, G. W., Nam, J.-W. \& Bartel, D. P. Predicting effective microRNA target sites in mammalian mRNAs. Elife 4, (2015). 
41. Artavanis-Tsakonas, S. Notch Signaling: Cell Fate Control and Signal Integration in Development. Science (80-. ). 284, 770-776 (1999).

42. Bray, S. J. Notch signalling in context. Nat. Rev. Mol. Cell Biol. 17, 722-735 (2016).

43. Kopan, R. \& Ilagan, M. X. G. The Canonical Notch Signaling Pathway: Unfolding the Activation Mechanism. Cell 137, 216-33 (2009).

44. Iso, T., Kedes, L. \& Hamamori, Y. HES and HERP families: Multiple effectors of the notch signaling pathway. J. Cell. Physiol. 194, 237-255 (2003).

45. Lamar, E. et al. Nrarp is a novel intracellular component of the Notch signaling pathway. Genes Dev. 15, 1885-1899 (2001).

46. del Álamo, D., Rouault, H. \& Schweisguth, F. Mechanism and significance of cis-inhibition in Notch signalling. Curr. Biol. 21, R40-7 (2011).

47. Borczuk, A. C. Assessment of invasion in lung adenocarcinoma classification, including adenocarcinoma in situ and minimally invasive adenocarcinoma. Mod. Pathol. 25, S1S10 (2012).

48. Laughney, A. M. et al. Regenerative lineages and immune-mediated pruning in lung cancer metastasis. Nat. Med. 26, 259-269 (2020).

49. Nabhan, A. N., Brownfield, D. G., Harbury, P. B., Krasnow, M. A. \& Desai, T. J. Singlecell Wnt signaling niches maintain stemness of alveolar type 2 cells. Science (80-. ). 359, 1118-1123 (2018).

50. Tammela, T. et al. A Wnt-producing niche drives proliferative potential and progression in lung adenocarcinoma. Nature 545, 355-359 (2017).

51. Liu, T. et al. Notch1 Signaling in FIZZ1 Induction of Myofibroblast Differentiation. Am. J. Pathol. 174, 1745-1755 (2009).

52. Strell, C. et al. Impact of Epithelial-Stromal Interactions on Peritumoral Fibroblasts in Ductal Carcinoma in Situ. JNCI J. Natl. Cancer Inst. 111, 983-995 (2019).

53. Su, Q. et al. Jagged1 upregulation in prostate epithelial cells promotes formation of reactive stroma in the Pten null mouse model for prostate cancer. Oncogene 36, 618-627 (2017).

54. Hu, B. et al. Multifocal Epithelial Tumors and Field Cancerization from Loss of Mesenchymal CSL Signaling. Cell 149, 1207-1220 (2012). 
55. Procopio, M.-G. et al. Combined CSL and p53 downregulation promotes cancerassociated fibroblast activation. Nat. Cell Biol. 17, 1193-1204 (2015).

56. Caswell, D. R. et al. Obligate progression precedes lung adenocarcinoma dissemination. Cancer Discov. 4, 781-9 (2014).

57. Muzumdar, M. D. et al. A global double-fluorescent cre reporter mouse. Genesis 45, 593605 (2007).

58. Seluanov, A., Vaidya, A. \& Gorbunova, V. Establishing primary adult fibroblast cultures from rodents. J. Vis. Exp. (2010). doi:10.3791/2033

59. Okada, N. et al. A positive feedback between p53 and miR-34 miRNAs mediates tumor suppression. Genes Dev. 28, 438-50 (2014).

60. Debnath, J., Muthuswamy, S. K. \& Brugge, J. S. Morphogenesis and oncogenesis of MCF-10A mammary epithelial acini grown in three-dimensional basement membrane cultures. Methods 30, 256-268 (2003).

61. Liu, Z. et al. Negative enrichment by immunomagnetic nanobeads for unbiased characterization of circulating tumor cells from peripheral blood of cancer patients. J. Transl. Med. 9, 70 (2011).

62. Fellmann, C. et al. An Optimized microRNA Backbone for Effective Single-Copy RNAi. Cell Rep. 5, 1704-1713 (2013).

63. Pelossof, R. et al. Prediction of potent shRNAs with a sequential classification algorithm. Nat. Biotechnol. 35, 350-353 (2017). 


\section{Material and Methods}

\section{Animals}

Kras $^{\text {LSL-G12D/+ }} ; \operatorname{Trp} 53^{\text {flox/flox }} ; R 26^{\text {LSL-tdTomato/+ }}$ mice have been described previously ${ }^{12,14,56}$. miR200c/141 and miR-200b/200a/429 null mice were acquired from The Wellcome Trust Sanger Institute ${ }^{16}$. Mice of $\mathrm{Kras}^{\mathrm{LSL}-\mathrm{G} 12 \mathrm{D} /+} ; \operatorname{Trp}^{\text {fllox/flox }}$ genotype were crossed with mir-200c/141/- mice to generate Kras ${ }^{\mathrm{LSL-G12D/+}} ; \operatorname{Trp5}^{\text {flox/flox}} ;$ mir-200c/141/- $($ KP200c) mice. miR-200c/141/acz mice were acquired from MMRRC and Michael McManus $l a b^{26}$, they were crossed with mice expressing Flippase (ACTB:FLPe B6J, JAX) to generate miR-200c/141 flox alleles. KP200c genotype were crossed with mir-200c/141 $1^{\text {floxfllox }}$ mice to generate Kras ${ }^{\text {LLL-G12D/+ }} ; \operatorname{Trp} 53^{\text {flox/flox }}$; mir-200c/141 ${ }^{\text {flox/- }}$ (KP200cCKO) mice. Lung tumorigenesis was induced by intranasal instillation of $5 \times 10^{6} \mathrm{PFU}$ of recombinant Ad-Cre virus (University of lowa Gene Transfer Vector Core) or by intratracheal intubation of $1 \times 10^{5} \mathrm{PFU}$ of Lenti-Cre in 8-week-old mice ${ }^{13}$.

\section{Human primary and metastatic tumor samples of lung adenocarcinoma}

Human tumor and blood samples were collected from patients approved by the Institutional Review Board of Shanghai Pulmonary Hospital, School of Medicine, Tongji University. The eight pairs of matched primary and metastatic tumor samples were collected from patients who were diagnosed with clinical stage Ila/llb/llla, pathologically confirmed lung adenocarcinoma and underwent pulmonary lobectomy and lymphadenectomy at Shanghai Pulmonary Hospital. The two liver metastases were obtained from palliative surgical resection of relapse lung adenocarcinomas. The samples of early-stage lung cancer were obtained from patients with stage la adenocarcinoma by lobectomy or segmentectomy. The patients' tumor tissues were made into FFPE blocks and subjected to H\&E staining or IHC routinely. The blood samples for isolating circulating tumor cell clusters were collected from patients with lung adenocarcinoma at stage IV.

\section{Isolation of mouse tumor cells and mouse lung fibroblast}

tdTomato+ tumor cells for miRNA profiling were collected from KPT mice by flow sorting as described previously ${ }^{56}$.

Cell lines were derived from individual tumors from KP and KP200c mice from 4 months after tumor initiation, and created by collagenase I (20U/ml, Worthington Biochemical)/ collagenase IV (800U/ml, Worthington Biochemical) digestion and mechanical dissociation. 
Normal lung fibroblast expressing tdTomato were collected from $R O S A^{m T / m G}$ mice ${ }^{57}$ using protocol described previously ${ }^{58}$ with modifications. Following lung harvest, the tissue was cut into small pieces and lightly digested with Liberase, and then cultured on scratched surface in Dulbecco's modified Eagle's medium (DMEM) supplemented with $10 \%$ fetal bovine serum (FBS), 100 units $/ \mathrm{ml}$ penicillin, $100 \mu \mathrm{g} / \mathrm{ml}$ streptomycin and $0.25 \mu \mathrm{g} / \mathrm{ml}$ amphotericin B (serumfree medium). Fibroblasts are highly migratory and proliferative compared to other cell types, they will attach to scratching marks and form outgrowth after 1 week. The fibroblast will be expanded and used 3-7 passages following primary culture.

\section{microRNA assays}

Total RNA was extracted from isolated tdTomato+ KPT tumor cells using TRIzol (Thermo Fisher Scientific) according to manufacturer's instruction. For Taqman microRNA assay, cDNA was synthesized with preamplification from total RNA using Megaplex ${ }^{\mathrm{TM}}$ RT Primers, Rodent Pool B v3.0 (Applied Biosystems ${ }^{\text {TM }}$, 4444292), Megaplex ${ }^{\text {TM }}$ PreAmp Primers, Rodent Pool B v3.0 (Applied Biosystems $\left.{ }^{\mathrm{TM}}, 4444308\right)$, TaqMan ${ }^{\circledR}$ MicroRNA Reverse Transcription Kit and TaqMan $^{\text {TM }}$ PreAmp Master Mix Kit according to manufacturer's instruction. Subsequently, quantitative-PCR was carried out using TaqMan ${ }^{\mathrm{TM}}$ Array Rodent MicroRNA A+B Cards Set v3.0 (Applied Biosystems ${ }^{\mathrm{TM}}$, 4444909). The data were analyzed using ViiA 7 Software and $\mathrm{R}$ program. Individual microRNA and mRNA were analyzed as previously described ${ }^{59}$. The primers used here are shown in Supplemental Table S3.

\section{Gene expression analysis of the TCGA data set}

LUAD data was downloaded from TCGA and normalized using upper quantile. All patients were into two groups - "lower" and "higher", according to the expression of the mir-200 family. The "lower" group included patients for whom all five mir200 expression values were lower than or equal to the median expression value of the corresponding mir200s in the normal control samples. The "higher" group included the rest of patients.

Survival analysis was performed to compare these two groups, as shown in Figure 1E, using the package "survival" in R (https://cran.r-project.org/web/packages/survival/index.html). Pvalue was determined by Log rank test, to quantify the significance of the differences in survival time between the two patient groups. 


\section{Three-dimensional tumor organoid culture for tumor-stromal interaction}

Lung tumor cells and primary lung fibroblast were routinely cultured in DMEM supplemented with $10 \%$ FBS, 100 units $/ \mathrm{ml}$ penicillin, $100 \mu \mathrm{g} / \mathrm{ml}$ streptomycin. The tumor organoid culture was performed using protocol described previously ${ }^{60}$ with modifications. 8-well chamber slides were coated with 100 ul Matrigel (354234, Corning)/ Collagen I $(0.5 \mathrm{mg} / \mathrm{ml}$, 354236, Corning) mixture. The slide was incubated at $37^{\circ} \mathrm{C}$ for 30 minutes to allow the mixture to solidify. Tumor cells and fibroblast cells were trypsinized and resuspended in growth media with supplement at $2 \times$ desired density and mix with equal volume of $10 \%$ Matrigel/Collagen I mixture. The cell/gel mixture were immediately plated on top of the matrix coating and cultured at $37^{\circ} \mathrm{C}, 5 \% \mathrm{CO}_{2}$. From day 2 to day $8,3 \mathrm{D}$ structures were monitored for invasive structure.

\section{Orthotopic transplantation experiment}

Tumor cells and fibroblast were co-cultured in suspension for 5 days prior to transplantation experiment. To prepare the suspension culture, the dissociated tumor cells and fibroblast are mixed at $1: 5$ ratio and seeded at $5 \times 10^{4} \mathrm{cells} / \mathrm{ml}$ density in ultra-low attachment dishes (Corning, CLS3262) in DMEM/F12 supplemented with human recombinant EGF (20 ng/mL, BioLegend), bFGF (20 ng/mL, PeproTech), B27 (Gibco by ThermoFisher) and 4 $\mathrm{g} / \mathrm{mL}$ Heparin (StemCell Technology). On the day of transplantation, tumor organoids were dissociated into single cells using trypsin/EDTA, and the percentage of tumor cells and tdTomato+ fibroblasts was determined by FACS. Single cell suspension containing $1 \times 10^{5}$ tumor cells were resuspended in $50 \mu l$ RPMI 1640 with 10mM EDTA and injected into the lung of nu/nu mice (Taconic) by intratracheal intubation. The tumors were collected either at 40 days after the tumor injection or at their terminal stage. The University of California Berkeley Animal Care and Use Committee approved all animal studies and procedures.

\section{Histopathology and immunohistochemistry}

For determining tumor histology, whole lungs were fixed overnight in $10 \%$ buffered formalin (Fisher Scientific, no. SF100-4), dehydrated in a graded series of ethanol solutions, then embedded in Paraffin Wax (ACROS Organics). The lung blocks were sectioned at $5 \mu \mathrm{m}$ thicknesses, mounted on glass slides, and stained with hematoxylin and eosin (H\&E) using standard procedures. Lung and tumor areas were determined using Image Viewer (Ventana Medical Systems/Roche). Each tumor was given a score of grade $1-5^{14}$. Grades 1 and 2 were classified as low-grade tumors, and grades 3-5 were classified as high-grade tumors. 
For IHC, paraffin sections were deparaffinized, dehydrated, and subjected to heat-induced antigen retrieval in a pressure cooker using Trilogy ${ }^{\mathrm{TM}}$ (Cell Marque, 920P). Slides were incubated for 10 min with $3 \% \mathrm{H}_{2} \mathrm{O}_{2}$, blocked for $1 \mathrm{~h}$ with $10 \%$ goat serum in phosphate-buffered saline (PBS) containing $0.05 \%$ Triton X-100, and incubated with primary antibody overnight in blocking buffer. Horseradish peroxidase (HRP)-conjugated secondary antibodies were incubated for $2 \mathrm{~h}$ at room temperature, with a 1:400 dilution in blocking buffer. For CC10, Nkx2.1 and p-Erk staining, a biotinylated horse anti-rabbit secondary antibody (Jackson ImmunoResearch, 711-065-152) was used at 1:400 as above, followed by incubation with Vectastain elite ABC reagent (Vector Laboratories, PK-7100) for 30 min. Peroxidase was then visualized by DAB staining (Vector Laboratories, SK-4100) and counterstaining with Gill's hematoxylin solution.

\section{Detection of circulating tumor cell clusters}

The blood for circulating tumor cell clusters detection was obtained from patients with stage IV lung adenocarcinoma. The previously described subtractive enrichment approach ${ }^{61}$ was modified to detect circulating tumor cell clusters. Briefly, $5 \mathrm{~mL}$ heparin-anticoagulated blood was added with 10 volume of Tris-buffered $\mathrm{NH}_{4} \mathrm{Cl}$ for erythrocyte lysis, and centrifuged at $500 \mathrm{~g}$ for $10 \mathrm{~min}$. The pellets were resuspended in $0.2 \mathrm{~mL}$ PBS and re-lysed with $1 \mathrm{~mL}$ OptiLyse $\mathrm{C}$ (Beckman Coulter) for $15 \mathrm{~min}$. After centrifugation, the pellets were dispersed and fixed in 1\% paraformaldehyde PBS for $10 \mathrm{~min}$, washed and resuspended in $0.5 \mathrm{~mL} 0.1 \%$ BSA PBS. $0.5 \mathrm{~mL}$ CD45 Dynabeads (ThermoFisher scientific) was added to deplete leukocyte fraction according to the manufactory's instruction. The CD45-depleted fraction was smeared on 3 positive charged slides. Cell clusters were observed and labeled on microscope for the following H\&E or immunofluorescent staining.

\section{Antibodies}

The following monoclonal $(\mathrm{mAb})$ and polyclonal $(\mathrm{pAb})$ primary antibody were used for immunoblotting, IF and IHC: Prosurfactant protein (SPC) pAb (Millipore, AB3786), Uteroglobin (CC10) pAB (Millipore, ABS1673), pErk1/2 pAb (Cell Signaling Technology, 4370), TTF1 (Nkx2.1) pAb (Abcam, ab76013), activated Notch pAb (Abcam, ab8925), Jag1 mAb (Cell Signaling Technology, 70109), a-SMA mAb (Invitrogen, 14-9760-82), Ki67 mAb (Cell Signaling Technology, 12202), RFP pAb (Rockland, 600-401-379). 


\section{Jag1/Jag2 knockdown}

The Jag1 knockdown lentiviral expression vector were constructed into pRRL-SFFV-GFP-miRE-Puro (SGEP) vector and the Jag2 knockdown lentiviral expression vector were constructed into pRRL-SFFV-GFP-miR-E-Neo (SGEN) vector62. The oligo sequences encoding Jag1 and Jag2 shRNA were designed using splashRNA algorithm ${ }^{63}$ (Supplementary Table S4), and cloned into Xhol/EcoRI sites. The shRNA lentiviral vectors were package in HEK293 cells together with pVSV-G and pMD-2 as previously described. Five shRNAs were tested for each gene, and the ones with best knockdown effect were used to generated double knockdown cell line. Cells transduced with both shJag1 and shJag2 were selected $48 \mathrm{hr}$ post infection using $2 \mu \mathrm{g} / \mathrm{ml}$ puromycin (Gibco by ThermoFisher) and $500 \mu \mathrm{g} / \mathrm{ml}$ Geneticin (Gibco by ThermoFisher). 


\section{Figure Legend}

Figure 1. miR-200 deficiency induces rapid metastasis in KP lung cancer model. A. Cartoon illustrating isolation of tdTomato+ cancer cells from primary and metastatic KPT lung adenocarcinomas for miRNA profiling. B. miR-200 miRNAs are strongly downregulated in distant metastases in the KPT mouse lung adenocarcinoma model. A Heatmap is shown for the most differentially expressed miRNAs between seven KPT primary lung tumors and four distant metastases $(P<0.05$ \& Expression level $\geq-20)$. Red text, miR-200 miRNAs. C. The five miR-200 miRNAs reside in two genomic loci and segregate into two sub-families based on seed sequences. Red box, seed sequences. D. miR-200 miRNAs are strongly downregulated in metastatic KPT tumors. Real time PCR detected miR-200 expression in KP early-stage primary tumor $(\mathrm{N}=2)$ and late-stage primary tumor $(\mathrm{n}=10)$, but not in $K P$ metastases $(\mathrm{n}=7)$. Error bars, s.e.m.; late stage primary tumors vs. metastases: $m i R-200 a,{ }^{* *} P=0.0052, \mathrm{t}=3.268, \mathrm{df}=15$; $\operatorname{miR}$ 429: * $P=0.0113, \mathrm{t}=2.887, \mathrm{df}=15 ; \operatorname{miR}-141$ : ** $P=0.01, \mathrm{t}=2.047, \mathrm{df}=15 ;$ miR-200c: ** $P=0.0068$, $\mathrm{t}=3.395, \mathrm{df}=10$. All statistical analyses were performed using unpaired, two tailed, student's $\mathrm{t}-$ test. E. Decreased total MIR-200 expression human lung adenocarcinomas is associated with poor patient survival in the TCGA data (LUAD). A Kaplan-Meier plot compares patient survival between two cohort of patients, with either high $(\mathrm{N}=410)$ or low $(\mathrm{N}=22)$ expression of all five MIR-200 miRNAs. ( ${ }^{* *} P=0.00188$, log rank test). F. miR-200c/141 deficiency in the KP200c model significantly reduces overall survival. A Kaplan-Meier plot compares survival of $K P$ and

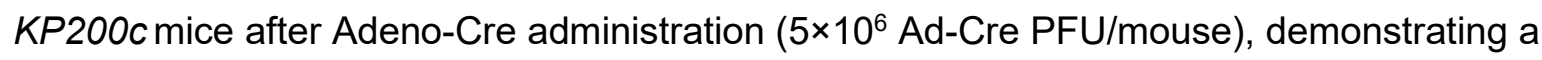
significant acceleration of tumor progression in $K P 200 \mathrm{c}$ mice. ${ }^{* * *} P=0.0003$, log rank test. G. miR-200c/141 deficiency in KP200c mice induces a significant increase in tumor progression. Representative H\&E staining of lung sections are shown for three pairs of $K P$ and $K P 200 c$ mice collected at 90 days after tumor initiation. Red arrows, lymph node metastases. H. KP200C primary tumors are characterized by desmoplastic stroma (blue arrows) and pleomorphic nuclei in cancer cells (red arrows). Representative images are shown for a pair of late-stage $K P$ and KP200c primary tumors collected at terminal stage. Scale bars, $100 \mu \mathrm{m} \mathrm{I.} \mathrm{miR-200} \mathrm{deficiency}$ leads to a significant increase in tumor burden, tumor size, and tumor grade in KP200c mice. Tumor burden is quantified as the percentage of tumor area versus total lung area (left, error bars, s.e.m. ${ }^{* * *} P<0.0001, \mathrm{~N}$, animal number, unpaired two-tailed Student's t-test, $\mathrm{t}=7.7222$, $\mathrm{df}=23$ ); tumor size was measured by tumor area (middle, error bars, s.e.m., ${ }^{* \star} P=0.006$, n, tumor number, unpaired two-tailed Student's t-test, $\mathrm{t}=2.792, \mathrm{df}=141$ ); tumor grade was determined by histopathological scores (right, $\mathrm{n}$, tumor number. ${ }^{* *} P<0.0001, \mathrm{x} 2=79.43, \mathrm{df}=2$ ). All $K P$ and 
KP200c tumors were collected at 12 weeks after tumor initiation. J. KP200c mice exhibit a greater metastasis frequency compared to KP mice. $26 \mathrm{KP}$ and $32 \mathrm{KP} 200 \mathrm{c}$ mice were compared upon tumor initiation with high-dose Cre virus $\left(5 \times 10^{6}\right.$ Ad-Cre PFU/mouse, *** $P<0.0001, \mathrm{X} 2=149.3, \mathrm{df}=2$ ), with $K P 200 \mathrm{c}$ mice developing more $\mathrm{LN}$ metastases. $4 \mathrm{KP}$ and 6 KP200c mice were compared upon tumor initiation with low-dose Cre virus $\left(1 \times 10^{5}\right.$ Lenti-Cre PFU/mouse, ${ }^{* *} P<0.0001, \mathrm{x} 2=2428, \mathrm{df}=2$ ), with $K P 200 \mathrm{c}$ mice developing more distant metastases. K. KP200c mice develop metastases in multiple sites. Representative images of H\&E staining of a lymph node metastasis, a pleural metastasis, and a distant metastasis in adrenal gland from KP200c mice are shown. Dotted line shows the boundary of metastasis and normal tissue. Scale bar, $100 \mu \mathrm{m}$.

Figure 2. CAFs are enriched in metastatic KP200c lung adenocarcinomas. A. Primary and metastatic KP200c tumors exhibit a strong increase in MAPK signaling. Immunohistochemistry staining of phosphorylated Erk1/2 (p-Erk) (left) and quantitation of p-Erk-positive cells (right) indicate a strong elevation of MAPK signaling in KP200c tumors, particularly the metastatic KP200c tumors. 27 KP primary tumors, 40 KP200c primary tumors and 6 KP200c metastases were analyzed. Scale bar, $100 \mu \mathrm{m}$. Error bar, s.e.m., $K P$ vs. $K P 200 \mathrm{c}$ primary tumor, ${ }^{\star} P=0.0263$, unpaired two-tailed Student's $\mathrm{t}$-test, $\mathrm{t}=2.273$, $\mathrm{df}=65 ; \mathrm{KP} 200 \mathrm{c}$ primary vs KP200c lymph node metastases, ${ }^{* * *} P<0.0001$, unpaired two-tailed Student's t-test, $\mathrm{t}=11.06, \mathrm{df}=44$. B. Metastatic KP200c tumors are characterized by a loss of cell lineage markers. Quantitation is shown for immunohistochemistry staining of CC10 (a marker for Club cells) and SPC (a marker for alveolar type 2 cells) in KP200c primary and metastatic tumors (left). Tumor stratification shows a preferential loss of CC10 and SPC expression in KP200c metastases compared to KP200c primary tumors. KP200c primary tumors vs. metastases ${ }^{* *} P<0.0001, \mathrm{x} 2=61.67, \mathrm{df}=3$. C. Metastatic KP200c tumors exhibit a downregulation of Nkx2. Representative images (left) and quantitation (right) of Nkx2.1 immunohistochemistry staining are shown for KP200c primary

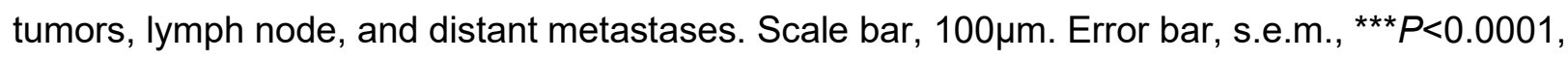
unpaired two-tailed Student's t-test, $\mathrm{t}=9.474$, $\mathrm{df}=57.47 \mathrm{KP} 200 \mathrm{c}$ primary tumors and $12 \mathrm{KP} 200 \mathrm{c}$ metastases (both lymph nodeand distant metastases) were analyzed. D, E. a-SMA-expressing CAFs are enriched in KP200c primary tumors and metastases. D. Representative images of $\mathrm{H} \& \mathrm{E}$ and $\alpha$-SMA staining are shown high grade regions of $K P$ and $K P 200 c$ primary tumors, as

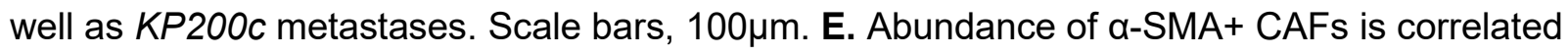
with the size of KP200c metastases. The percentage of a-SMA staining in total tumor area is plotted in a linear regression against the size of metastatic tumors for $10 \mathrm{KP} 200 \mathrm{c}$ lymph node 
metastases, $R^{2}=0.3599$. F. $\alpha$-SMA positive fibroblasts are absent from $K P$ and $K P 200 c$ normal lung and early primary tumors. Representative images of $\alpha$-SMA staining are shown for $K P$ and KP200c normal lung and early primary tumors. Scale bars, $100 \mu \mathrm{m}$. G. Deficiency of miR200c/141 specifically in cancer cells promotes metastasis progression and desmoplastic stroma. Left, KP200cCKO lungs collected 90 days after tumor initiation ( $5 \times 10^{6}$ Adeno-Cre PFU/mouse) exhibit evident lymph node metastases, with metastasis frequency comparable to that of $K P$ mice. Right, $\mathrm{H} \& E$ and $\alpha-S M A$ staining of high grade regions of KP200cCKO primary tumors and lymph node metastases indicate strong CAF enrichment in the tumor microenvironment. Scale bars, $100 \mu \mathrm{m}$. H, I. Human metastatic lung adenocarcinomas exhibit an increase of $\alpha-S M A+$ CAFs compared to paired primary tumors. H. Quantitation of $\alpha-S M A+$ area was shown for 8 pairs of human primary and metastatic lung adenocarcinomas; representative

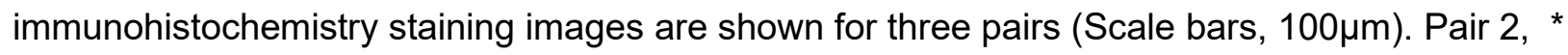
$P=0.0148, \mathrm{t}=4.101, \mathrm{df}=4.000$; pair $3^{* * *} P=0.0004, \mathrm{t}=11.22, \mathrm{df}=4.000$; pair 4 * $P=0.0467$, $\mathrm{t}=2.883, \mathrm{df}=3.869$; pair 5, ${ }^{* *} P=0.0010, \mathrm{t}=6.955, \mathrm{df}=4.951$; pair $6{ }^{*} P=0.0250, \mathrm{t}=3.530$, $\mathrm{df}=3.920$; pair $7^{* * *} P=0.0001, \mathrm{t}=27.80, \mathrm{df}=3.000$; pair $8^{* * *} P<0.0001, \mathrm{t}=19.42, \mathrm{df}=4.000$. All statistical analyses were performed using unpaired two-tailed Student's t-test with Welch's correction. I. a-SMA+ CAFs are enriched in liver metastases from relapsed human lung adenocarcinoma patients. H\&E staining are shown for two independent liver metastases samples and $\alpha$-SMA staining are shown for the boxed metastasis region. Dotted line indicates the tumor/liver boundary. Scale bars, $100 \mu \mathrm{m}$. J. Increased cancer-CAF interaction is associated with tumor progression in human lung adenocarcinomas. A stage IA lung adenocarcinoma was stained for $\alpha-S M A$, regions representing a progressive histopathological pattern including normal lung, atypical adenomatous hyperplasia (AAH), and adenocarcinoma (ADC) are marked and zoom in. Scale bar, $100 \mu \mathrm{m}$.

\section{Figure 3. miR-200 deficiency in cancer cells promote CAF activation to drive tumor}

metastasis. A. A diagram illustrating the tumor-fibroblast organoid co-culture model. GFP labeled KP or KP200c lung cancer cells are co-cultured with tdTomato labeled normal lung fibroblasts to form tumor organoids. B, C, D. miR-200 deficiency in KP cancer cells induces metastatic features upon co-culture with lung fibroblasts. B. Fluorescence images (left) and quantitation (right) of tumor-fibroblast organoids indicate that $m i R-200$ deficiency in $K P$ cancer cells, combined with fibroblast co-culture, induced tumor clustering and promoted metastatic cellular features. Scale bar, $100 \mu \mathrm{m}$. KP alone: $\mathrm{n}=283, K P+F$ ibroblasts: $\mathrm{n}=237, K P 200 c$ alone: $\mathrm{n}=317, K P 200 c+$ Fibroblasts: $\mathrm{n}=256 . K P 200 c$ alone vs. KP200c+Fibroblasts, ${ }^{* * *} P<0.0001$, 
$\mathrm{t}=7.404, \mathrm{df}=568 ; K P 200 \mathrm{c}+$ Fibroblasts vs. $K P+$ Fibroblasts, ${ }^{* * *} P<0.0001, \mathrm{t}=7.894, \mathrm{df}=488$; unpaired two-tailed Student's t-test. C, D. Representative images and quantification are shown for clustering and invasiveness in KP and KP200c tumor organoids with fibroblast co-culture. Scale bars, $100 \mu \mathrm{m}$. C. KP200c organoids exhibit invasive features in cancer cells (arrows) and elongated morphology in co-cultured fibroblasts (arrowheads). D. Tumor organoids exhibit three morphologies: spherical organoids, large clusters, and large clusters with invasive cancer cell features. Analysis of $87 \mathrm{KP}$ and $112 \mathrm{KP} 200 \mathrm{c}$ tumor organoids indicate a strong increase in invasive features in KP200c tumor organoids. E. Ki67 immunostaining in KP200c organoid with fibroblast co-culture indicates strong cell proliferation in both cancer cells and fibroblasts

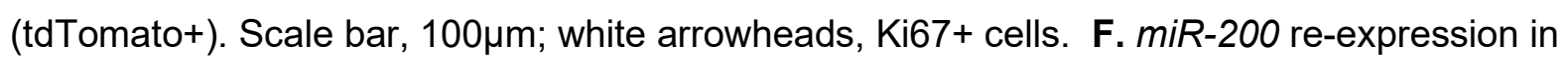
KP200c cancer cells decreases cell proliferation of tumor-associated lung fibroblasts in organoid co-culture, but fails to affect non-tumor associated fibroblasts. Density of tumor-associated fibroblasts and non-tumor-associated fibroblasts were quantified by measuring the tdTomato signal coverage. Error bar, s.d.. KP non-tumor-associated fibroblasts vs. tumor-associated fibroblasts, ns $P=0.3823, \mathrm{t}=1.021$, df=3; KP200c-MSCV non-tumor-associated fibroblasts vs. tumor-associated fibroblasts, ${ }^{* * *} P=0.0006, \mathrm{t}=6.484$, df=6; KP200c-MSCV-miR-200c/141 nontumor-associated fibroblasts vs. tumor-associated fibroblasts, $\mathrm{ns} P=0.2456, \mathrm{t}=1.440, \mathrm{df}=3$, unpaired two-tailed Student's t-test. G. Co-culture of KP200c cancer cells and lung fibroblasts in organoids promotes $\alpha-S M A$ induction in fibroblasts. Scale bars, $100 \mu m$. H. Increased $\alpha-S M A+$ CAF proliferation is observed in KP200c high-grade primary tumors and metastatic tumors, but not in KP200c MSCV low-grade primary tumors. Low-grade primary tumors, high-grade primary tumors, lymph node metastases, and adrenal gland metastases from KP200c mice were immunostained for Ki67 and $\alpha-S M A$. White arrowheads, proliferating CAFs (Ki-67+, $\alpha-S M A+)$. Scale bars, $20 \mu \mathrm{m}$. I. miR-200 expression in KP200c cancer cells regulates the induction of specific cytokine and growth factors by CAFs. Lung fibroblasts co-cultured with or without control KP200c organoids or miR-200 re-expressing KP200c organoids were isolated by flow cytometry, and $\mathrm{Hgf}$, Tgfb2, Fgf1 and Ccl5 levels were quantified by real time PCR. Control KP200c cancer cells enhanced fibroblast production of pro-metastatic cytokines and growth factors, while miR-200 re-expression in KP200c cancer cells reversed this phenotype. Error bar, s.d. All statistical analyses are performed using unpaired, two-tailed Student's t-test. J. K. KP200c cancer cells primed with lung fibroblasts yield highly metastatic lung tumors in an orthotropic allograft tumor model 4 weeks after tumor transplantation. J. A cartoon illustrating the orthotropic allograft model to study lung cancer metastasis using co-cultured KP200c cancer cells and lung fibroblasts. KP200c tumor organoids without or with tdTomato+ lung fibroblast co- 
culture at 1:5 ratio for 5 days were dissociated, and single cell suspension containing $1 \times 10^{5}$ cancer cells were subsequently transplanted to immune deficient $n u / n u$ recipient mice through intratracheal injection. K. H\&E staining reveals a highly metastatic tumor phenotype in primary tumors, lymph node metastases, and distant metastases generated from KP200c cancer cells primed by lung fibroblast co-culture. Scale bar, $500 \mu \mathrm{m}$, black arrowheads, metastases L. Transplanted exogenous fibroblasts and endogenous fibroblasts from recipient mice both contribute to CAFs in metastatic tumor microenvironment. Disseminating tumor clusters from pleural cavity and pleural metastasis both contain transplanted tdTomato+ CAFs and endogenous tdTomato- CAFs. Scale bar $=100 \mu \mathrm{m}$, red arrowheads, tdTomato+ CAFs, black arrowheads, tdTomato- CAFs.

\section{Figure 4. $m i R-200$ represses Jag1/Jag2 in cancer cells and inhibits Notch signaling in}

CAFs. A. Direct cell-cell contact mediates the activation of co-cultured fibroblasts by KP200c cancer cells. Fibroblasts and KP200c cancer cells were co-cultured either in the organoid coculture or in transwell assays, and the abundance of fibroblasts was measured either by the percentage of tdTomato+ signal (in organoid co-culture) or by OD595 of crystal violet staining (in transwell assay). KP200c cancer cells promote proliferation of fibroblasts only in tumor organoid culture, where direct cell-cell contact was permitted. Error bars, s.d., Fibroblasts + KP tumor organoids vs. Fibroblasts $+K P 200 c$ tumor organoids ${ }^{*} P=0.0104, \mathrm{t}=3.185, \mathrm{df}=2$, unpaired two-tailed Student's t-test. B. Candidate miR-141 and miR-200c binding sites in the 3' UTR of Jag1 and Jag2, respectively, as predicted by TargetScan. C. Metastatic KP200c tumors exhibit an induction of Jag1 and Jag2 compared to KP200c primary tumors. $10 \mathrm{KP} 200 \mathrm{c}$ primary tumors and 2 lymph node metastases were subjected to real time PCR for Jag1 and Jag2. Error bars, standard error. Jag1 KP200c primary tumor vs. LN met *** $P<0.0001, \mathrm{t}=12.03, \mathrm{df}=10$, Jag2 KP200c primary tumor vs. LN met * $P=0.0290, \mathrm{t}=2.548, \mathrm{df}=10$, unpaired Student's t-test. D. Overexpression of mir-200c/141 in KP200c cancer cells suppresses the mRNA (left) and protein (right) expression of both Jag1 and Jag2. Error bars, s.d.. E. Co-culture of lung fibroblasts and KP200c cancer cells induces Notch signaling in fibroblasts, but represses Notch signaling in cancer cells. tdTomato+ lung fibroblasts and GFP+ cancer cells were co-cultured as tumor organoids for 5 days, before each population was isolated using flow cytometry for real time PCR. Fibroblasts co-cultured with KP200c cancer cells exhibit an increase in Notch target genes (Hes1 and Nrarp) compared to those co-cultured with KP cancer cells. When co-cultured with fibroblasts, KP200c cancer cells exhibit a decrease in Notch target genes compared to KP cancer cells. Error bar, s.d.. F. miR-200 re-expression in KP200c cancer cells represses Notch 
targets Hes1 and Nrarp in co-cultured fibroblasts. Error bars, s.d.. All statistical analyses were performed using unpaired, two-tailed Student's t-test. G. Activated Notch signaling in fibroblasts promotes metastatic behavior of co-cultured, miR-200 expressing cancer cells. Lung fibroblasts expressing mouse NICD1, NICD3, or control pcDNA3 vector were co-cultured with mir-200c/141 expressing KP200c cancer cells, and invasive features of cancer cells were examined by light microscopy. Fibroblasts with activated Notch signaling, but not control fibroblasts, conferred a metastatic phenotype in miR-200 expressing KP200c cancer cells in organoid culture. Scale bars, $100 \mu \mathrm{m}$; red arrows, invasive cellular structures in cancer cells co-cultured with fibroblasts with activated Notch signaling. Morphology of tumor organoids was quantified as described in Fig. 3D. H. Metastatic KP200c mouse tumors and metastatic human lung adenocarcinomas both contain CAFs with strong expression of nuclear Notch1, compared to paired primary tumors. Scale bars, $100 \mu \mathrm{m}$. Red arrows point to the CAF stained positive for activated Notch1. I. Knockdown of both Jag1 and Jag2 in KP200c cancer cells inhibits invasiveness in cancer cells and proliferation in fibroblasts when cultured as tumor organoids with lung fibroblasts. GFP labeled KP200c cancer cells expressing a control vector or shRNAs against both Jag1 and Jag2 were co-cultured with tdTomato labeled lung fibroblasts. Invasive features of cancer cells and cell proliferation of fibroblasts were analyzed by fluorescence microscopy (left). Jag1 and Jag2 double knockdown in KP200c cancer cells, when co-cultured with lung fibroblasts, restores a spherical morphology of tumor organoids (left), reduces the size of tumor organoids (middle), and decreases the invasive features of tumor organoids (right). Scale bar, $100 \mu \mathrm{m}$. J. A model illustrating the role of $m i R-200$ miRNAs in lung cancer metastasis by regulating the interaction between cancer cells and fibroblasts. In miR-200-expressing cancer cells, miR-200 targets Jag1 and Jag2; hence, the cancer cells fail to activate Notch signaling in adjacent fibroblasts and lack metastatic potential. miR-200 deficiency in cancer cells de-represses Jag1 and Jag2, triggering Notch activation in neighboring CAFs, which promotes fibroblast proliferation and activation, ultimately enhancing the metastatic potential of the cancer cells. 


\section{Supplementary figure legend}

\section{Supplementary Figure S1 miR-200 deficiency induces rapid metastasis in KP lung} cancer model A. miR-200c and miR-141 are highly expressed in KPT primary tumors and significantly downregulated in metastases. A scatterplot shows the expression difference between the KP primary tumors and metastases (y axis, $\Delta \mathrm{Ct}_{K P T \cdot \text { met }}-\Delta \mathrm{Ct}_{K P T \text {.primary }}$ ) versus total miRNA expression level of KPT primary tumors and metastases ( $\mathrm{x}$ axis, $\left.\left[\Delta \mathrm{Ct}_{K P T \text {.primary }}+\Delta \mathrm{Ct}_{K P T \text {.met }}\right]\right)$. The miR-200 family members are labeled with color corresponding with their $P$ value range. B. Schematic illustration of mir-200c/141 targeting and knockout strategy. Two homologous arms flanking the mir-200c/141 locus mediate gene-specific targeting by homologous recombination. Targeting replaces mir-200c/141 with a selection cassette containing a PGK-driven puromycin selection marker and a truncated TK variant ( $\Delta$ tk) flanked by loxP sites. Future puromycin selection and crossing with Cre transgenic mice leads to removal of reporter to generate mir-200c/141 knockout mice. Red arrows indicate the position of the PCR primers used for genotyping. Bottom, homozygous deletion was confirmed by PCR. C. The appearance of pleomorphic nuclei in early stage KP200c primary tumor. Representative images of H\&E staining of lung sections from KP and KP200c mice 1 month after Adeno-Cre infection are shown. Scale bar, $100 \mu \mathrm{m}$. Arrows, cancer cells with pleomorphic nuclei. D. Loss of miR-200 expression in the KP200c primary tumors. miR-200c and miR-200a expression was measured by real time PCR in the KP primary tumors $(n=12), K P 200 c$ primary tumors $(n=11)$ and KP200c LN metastases ( $\mathrm{n}=4)$. Error bars, s.e.m. E. Occurrence of metastases in multiple sites in KP200c mice. Photos of LN metastasis, liver metastasis, and pleural metastases on the pleural membrane in KP200c mice are shown.

\section{Supplementary Figure S2 Characterization of desmoplasia phenotype in metastatic} KP200c lung adenocarcinomas. A. Loss of cell lineage markers in metastatic KP200c tumors. $\mathrm{H} \& \mathrm{E}$ and IHC staining of KP and KP200c low-grade and high-grade primary tumors and KP200C LN metastasis, pleural metastasis, and distant metastasis with lung lineage markers including CC10 (a marker for Club cells) and SPC (a marker for alveolar type 2 cells) are shown. The

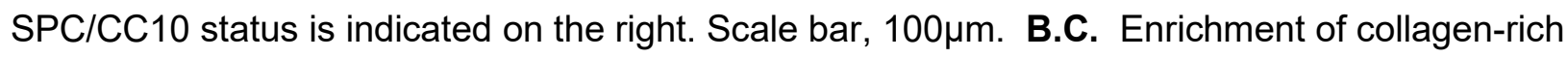
extracellular matrix in metastatic KP200c tumors. Representative images of B. Masson's trichrome staining and C. IHC staining for Collagen I of KP primary tumor, KP200c primary tumor, and KP200c metastasis are shown. In trichrome staining, collagenous fibers are stained 


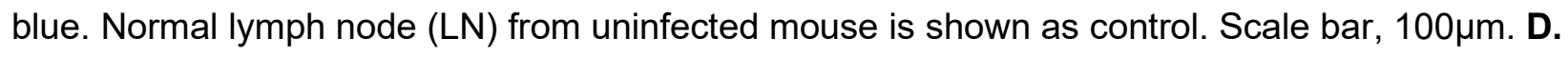
Lack of stromal desmoplasia in metastatic KP tumors. Representative images of H\&E staining of KP LN metastases, liver metastasis and KP200c LN metastases are shown. Scale bar, $100 \mu \mathrm{m}$. E. No change in FSP-1-expressing CAFs in metastatic KP200c tumors. Representative images of IHC staining for FSP-1 in KP primary tumor, KP200c primary tumor, and LN

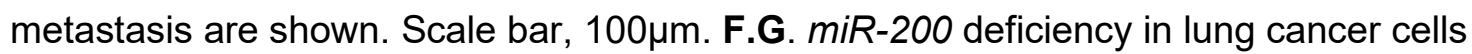
promotes local and distant metastases. F. Schematic illustration of mir-200c/141 targeting and generation of conditional knockout allele. Two homologous arms flanking the mir-200c/141 locus mediate gene-specific targeting by homologous recombination. Targeting leads to insertion of a cassette containing LacZ-polyA flanked by FRT and mir-141/200c flanked by loxP sites. Future neomycin selection and crossing with flippase transgenic mice leads to removal of reporter to generate a conditional mir-200c/141 knockout allele (mir-200c/141 flox $)$. Cre virus induces deletion of mir-200c/141 in infected cells. G. Kaplan-Meier survival curve of KP200c

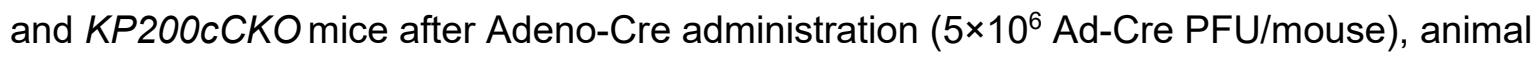
number $\mathrm{N}$ is indicated in the figure, Log rank Test ${ }^{*} P$-value $=0.0409$.

\section{Supplementary Figure S3 miR-200 deficiency promotes CAF activation and metastatic}

features in cancer cells. A. Loss of miR-200 expression in KP200c cancer cell lines.

Expression level of $m i R-200 c$ in one KP and three KP200c cancer cell lines was quantified by real time PCR, and U6 was used as housekeeping control. Error bar, s.d. B. Increased size of KP200c tumor organoids upon co-cultured with lung fibroblasts in multiple KP200c lines. One $K P$ and three KP200c cancer cell lines were co-cultured with or without lung fibroblasts, and tumorsphere size was measured by calculating the GFP (tumor) area coverage. More than 200 tumorspheres were measured and plotted for each line. Lines show the median with interquartile range. $\mathbf{C}$. The clustering behavior in KP200c organoids is proportional to the ratio of fibroblasts to cancer cells. Bright field images of KP200c cancer cells co-cultured with fibroblasts at ratios of $1: 1,1: 2,1: 5$, and 1:10 are shown. Scale bar, 100 $\mu \mathrm{m}$. D. Expression of $m i R-141$ and miR-200c in KP200c cell lines overexpressing MSCV control or MSCV-miR-200c/141 vector, as measured by real time PCR. U6 was used as housekeeping control. E. KPand KP200c cancer cells show no difference in growth rate in 2D culture. Same number of KP and KP200c cells were seeded in plates and subject to MTT assay at $24 \mathrm{hr}, 48 \mathrm{hr}$ and $72 \mathrm{hr}$. Each condition was repeated in triplicate. Error bar, standard error. F. Re-expression of $m i R-200 c / 141$ reverts the invasive phenotype in KP200c organoids. Representative bright field images overlaid with red 
channel fluorescence images of KP200c overexpressing MSCV control or MSCV-miR-200c/141

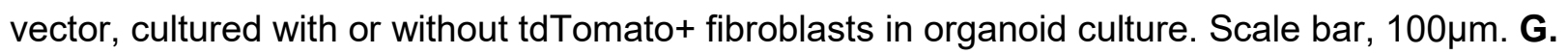
An Increase in CAF proliferation is observed in metastatic KP200c tumors. Low magnification image of low-grade, high-grade tumor, lymph node metastasis, and adrenal gland metastasis from KP200c animal stained for Ki67 and a-SMA. Arrows indicate proliferating CAFs that are

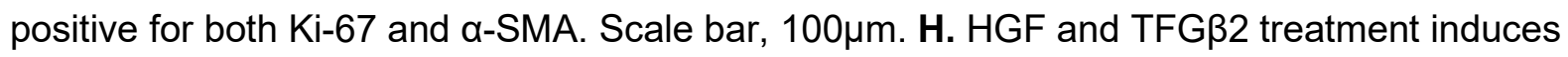
invasive phenotype in KP200c tumor organoids. Phase contrast image of KP200c cancer cells in organoid culture untreated or treated with HGF $(50 \mathrm{ng} / \mathrm{ml})$, TGF 2 (10 ng/ml), and a

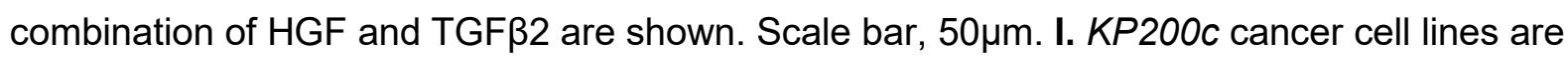
able to develop lung tumors in an orthotropic allograft lung tumor model. Two KP and three KP200c cancer cell lines were transplanted into the lungs of $n u / n u$ mice $\left(1 \times 10^{6} \mathrm{cells} / \mathrm{mouse}\right)$. Lungs were collected 7 weeks after transplantation and H\&E images of the lungs were shown. J. K. KP200c cancer cells primed with lung fibroblast yield highly metastatic lung tumors in an orthotropic allograft tumor model. J. Kaplan-Meier curve of mice transplanted with KP200c cancer cells co-cultured with or without lung fibroblasts. Animal number $\mathrm{N}$ is indicated in the figure, Log rank test ${ }^{* *} P=0.0062$. K. Photos of mice 4 weeks after transplantation with KP200c cancer cells co-cultured with or without fibroblasts. Red arrows indicate subcutaneous metastases in mice transplanted with KP200c cancer cells + fibroblasts. $L$. The presence of $\alpha-$ SMA+ CAFs with cancer cells inside blood/lymphatic vessel of KP200c lung. KP200c primary tumor was stained for $\alpha$-SMA, with the boxed area showing the presence of cancer cells accompanied by $\alpha-S M A+C A F s$ inside of the blood vessel. Red arrows indicate cancer cells, green arrows indicate $\alpha-S M A+C A F s$. C: Cancer cells V: blood/lymphatic vessel. Scale bar, $100 \mu \mathrm{m}$. M. $\alpha-S M A+C A F s$ adjacent to circulating cancer cells in pleural lavage in a human lung adenocarcinoma patient. Blood sample collected from a lung cancer patient was stained with anti-CK19 for cancer cells and anti- $\alpha$-SMA for CAFs. H\&E staining and fluorescence image are shown. Scale bar, $100 \mu \mathrm{m}$. N. The presence of $\alpha-S M A+C A F s$ during extravasation in a human minimally invasive adenocarcinoma sample (MIA). Red arrows indicate the minimum metastatic outgrowth encased in $\alpha-S M A+C A F s$ extravasating from the lymphatic vessel in the lymph node. Scale bar, 100um V: Lymphatic vessel, C: Cancer cells, L: Iymphocyte

\section{Supplementary Figure S4 miR-200-EMT is not required for cancer-CAF interaction and}

invasive behavior. A. KP200c tumors and metastases retain epithelial characteristics. Left, KP200c tumors and metastases retain membrane localization of E-cadherin. Representative images of E-cadherin staining of KP primary tumor, KP200c primary tumor, KP200c LN 
metastasis, and adrenal gland metastasis are shown. Zoom in area were shown. Scale bar, $100 \mu \mathrm{m}$. Right, expression of mesenchymal marker Vimentin and epithelial marker E-cadherin is not affected by miR-200c/141 expression in KP200c cancer cells, as measured by Western blot. $\beta$-tubulin was used as endogenous control. B. Upregulation of E-cadherin and $\mathbf{C}$. downregulation of Zeb1 and Zeb2 in KP200c tumor and metastases. mRNA from KP primary tumors $(n=5), K P 200 c$ primary tumor $(n=10)$ and LN metastases $(n=2)$ was collected and subjected to real time PCR. The expression level was normalized to $K P$ primary tumors. Error bar, standard error. $K P$ primary tumor vs. $K P 200$ c primary tumor, $C d h 1^{* * *} P=0.0009, \mathrm{t}=4.307$, $\mathrm{df}=13$, Zeb1 ${ }^{* *} P=0.0023, \mathrm{t}=3.857, \mathrm{df}=12, \mathrm{Zeb2}{ }^{* *} P=0,0055, \mathrm{t}=3.321, \mathrm{df}=13$, unpaired two tailed Student's t-test. D. Knockdown of Zeb1 or Zeb2 expression in KP200c cell line by shRNAs. For each gene, two shRNAs were used. E. Knockdown of Zeb1 or Zeb2 does not alter invasive morphology of KP200c cancer cells in 3D organoid co-culture. Phase contrast images of KP200c cancer cells expressing Zeb1 or Zeb2 shRNAs in organoid culture with or without

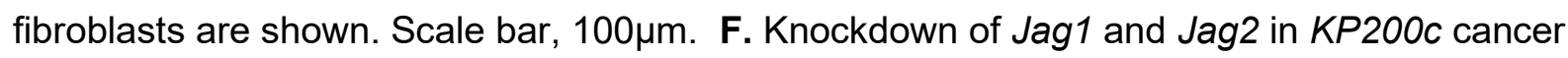
cells expressing double shRNAs against Jag1 and Jag2 was confirmed by real time PCR analysis. G. Activation of Notch in the metastases of lung cancer patients. Paired primary lung tumor and LN metastases from six lung adenocarcinoma patients were stained with anti-NICD1

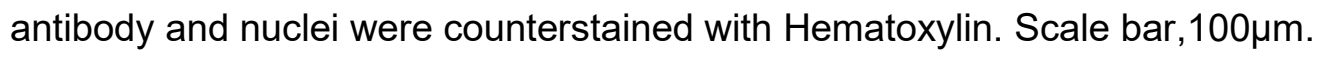

Supplementary Table $\mathbf{S 1}$ This table contains the miRNA expression data as $\triangle \mathrm{CT}$ (CT[miRNA of interest]-CT[housekeeping sRNAs]) for seven KPT primary tumors and four KPT distant metastases. Total miRNA expression level as $\triangle \mathrm{Ct}(K P T$.primary tumor $)+\triangle \mathrm{Ct}(K P T$.met $)$, fold change between $K P T$ primary tumors and distant metastases as $\triangle \mathrm{Ct}(K P T$.met)$\triangle \mathrm{Ct}(K P T$.primary tumor), and Student's test $P$-value are shown.

Supplementary Table S2 This table contains the metastasis ratio, metastasis sites and median survival time in the cohorts of KP, KP200c and KP200cCKO mice infected with highdose Cre virus (Adeno-Cre $5 \times 10^{6} \mathrm{PFU} / \mathrm{mouse}$ ) and low-dose Cre virus (Lenti-Cre $1 \times 10^{5}$ PFU/mouse).

Supplementary Table S3 Primer sequence of real time PCR on mouse samples.

Supplementary Table S4 Guide strand sequences for all shRNAs used in this study. 


\section{Figure 1}

A

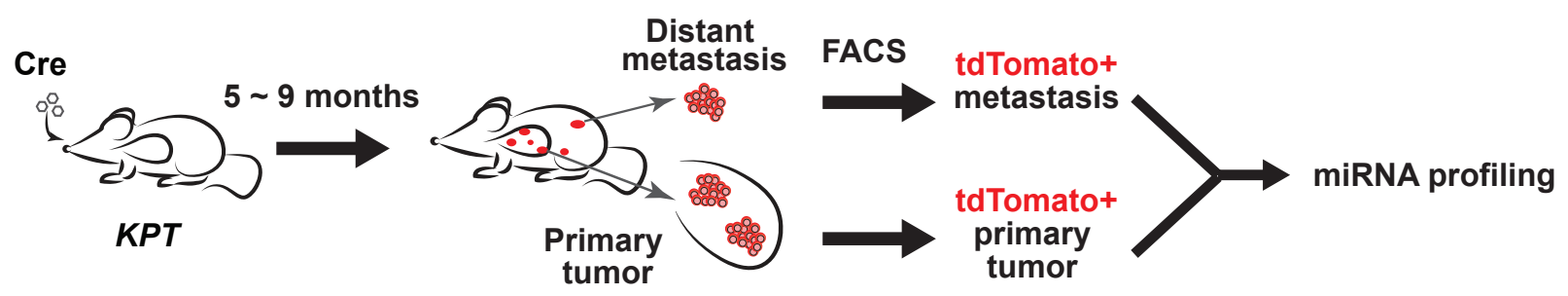

B
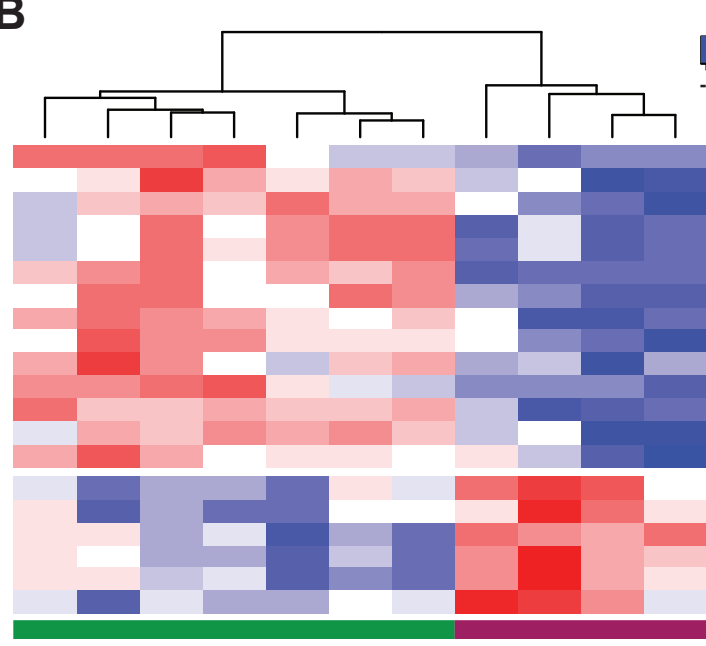

Primary tumor

Distant Metastasis

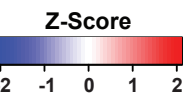

mmu-miR-1941-5p mmu-miR-376c-3p rno-miR-409a-3p mmu-miR-134-5p mmu-miR-667-3p mmu-miR-375-3p mmu-miR-485-3p mmu-miR-141-3 mmu-miR-146b mmu-miR-184-3p mmu-miR-146b-5p mmu-miR-200c-3p mmu-miR-434-3p mmu-miR-93-5p mmu-miR-805 mmu-miR-17-5p mmu-miR-106a-5p $m m u-m i R-18 a-5 p$
$m m u-m i R-23 b-3 p$
C

mir-200b/200a/429 (chr4-)

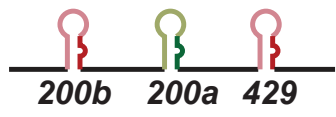

mir-200c/141 (chr6-)

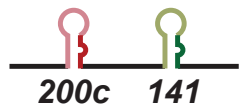

miR-200c miR-200b $m i R-429$

UAAUACGCCGGGUAUGAUGGA UAAUACUGCCUGGUAAUGAUGA. UAAUACUGUCUGGUAAUGCCGU -

miR-200a miR-141

UAACACUGUCUGGUAACGAUGU UAACACUGUCUGGUAAAGAUGG seed

D
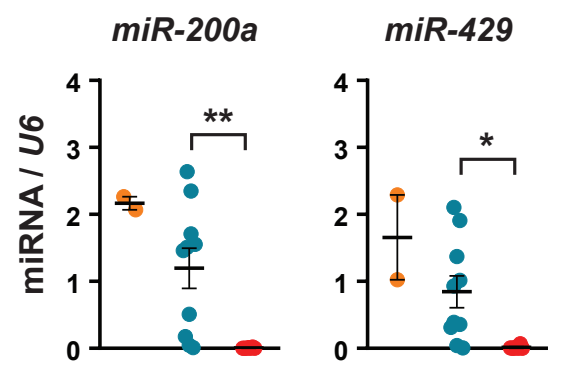

E

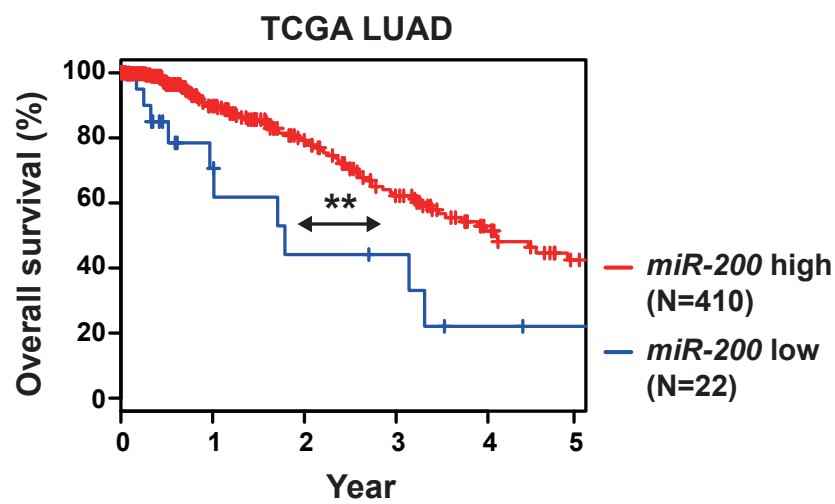

$\operatorname{miR}-141$

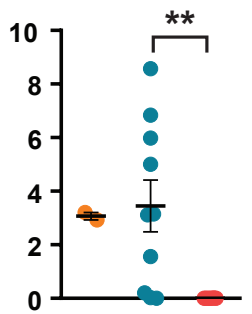

miR-200c

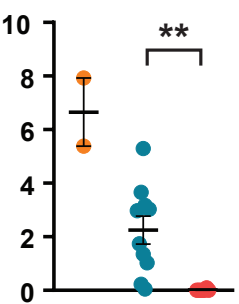

KPT Early stage primary tumor $(\mathrm{N}=2)$

KPT Late stage primary tumor $(n=10)$

KPT Distant metastasis $(\mathrm{n}=7)$

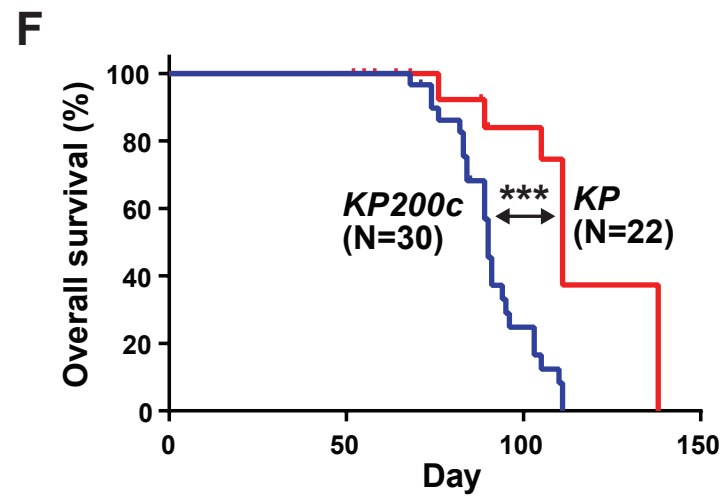




\section{Figure 1 cont'd}

G
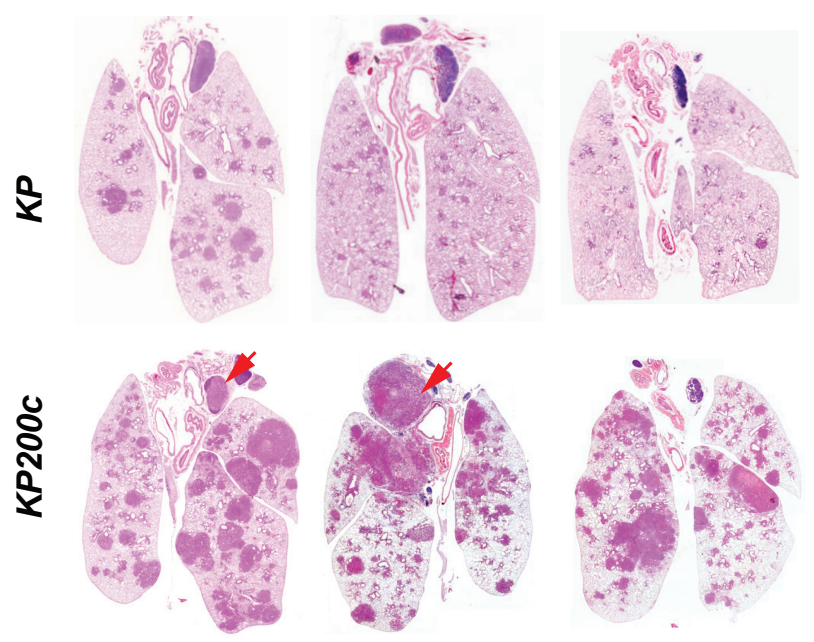

$\mathbf{H}$

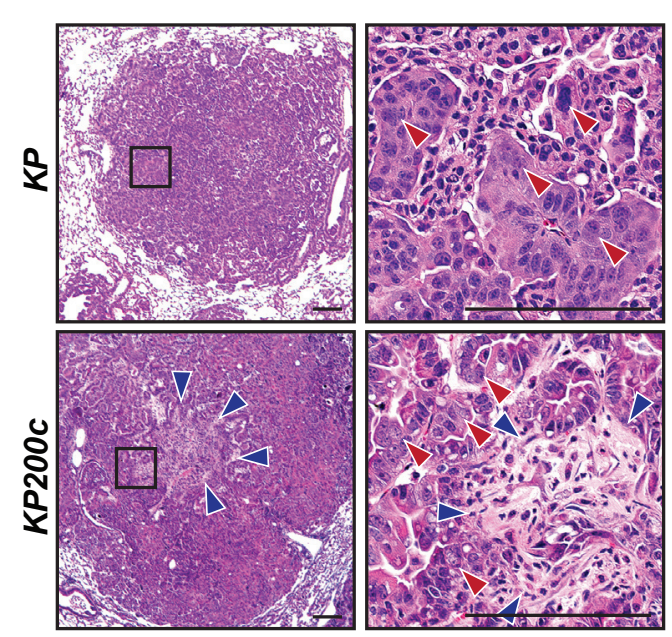

I
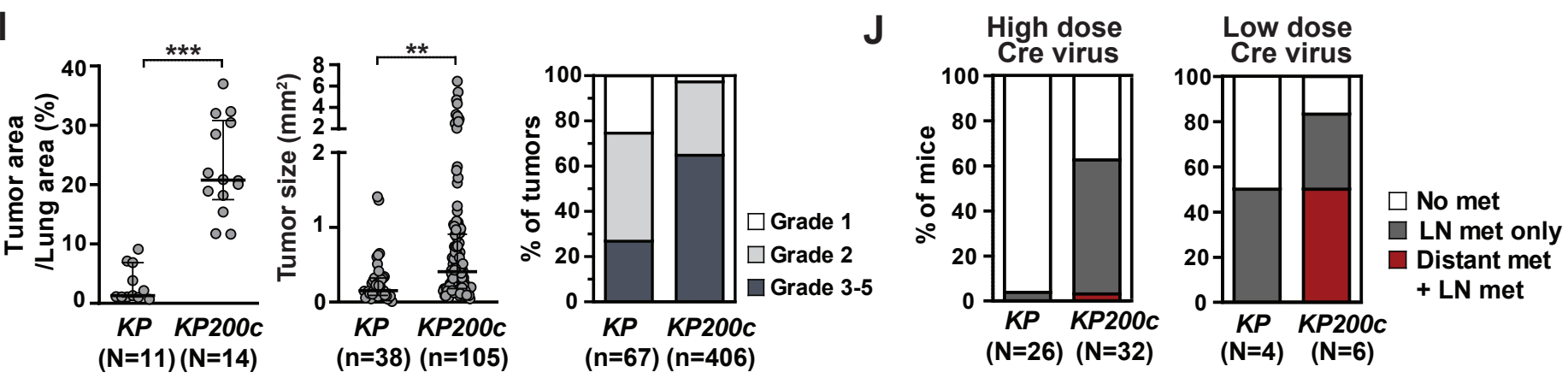

$\mathbf{K}$

KP200c metastases
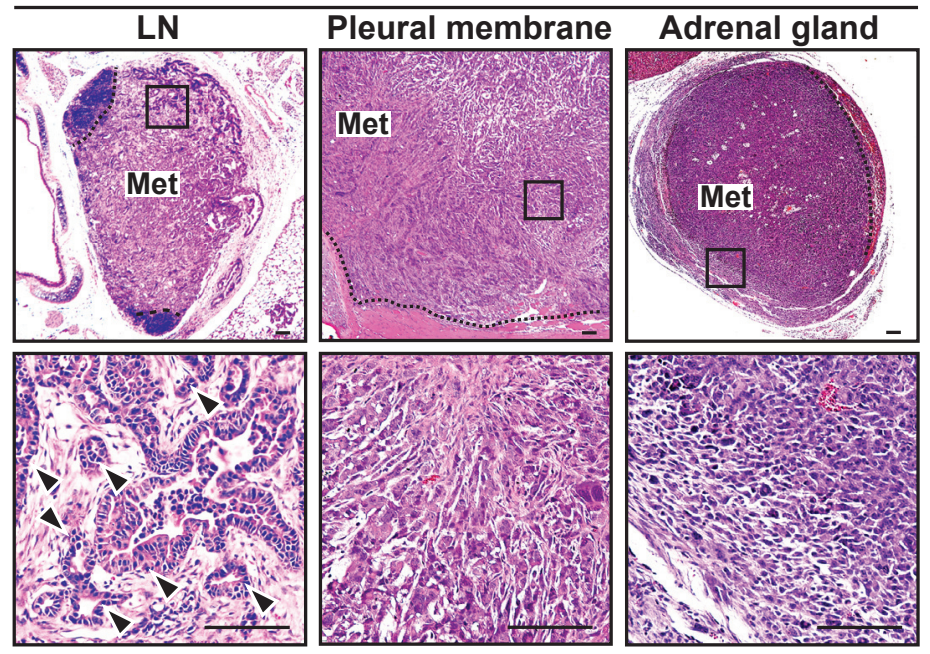


\section{Figure 2}
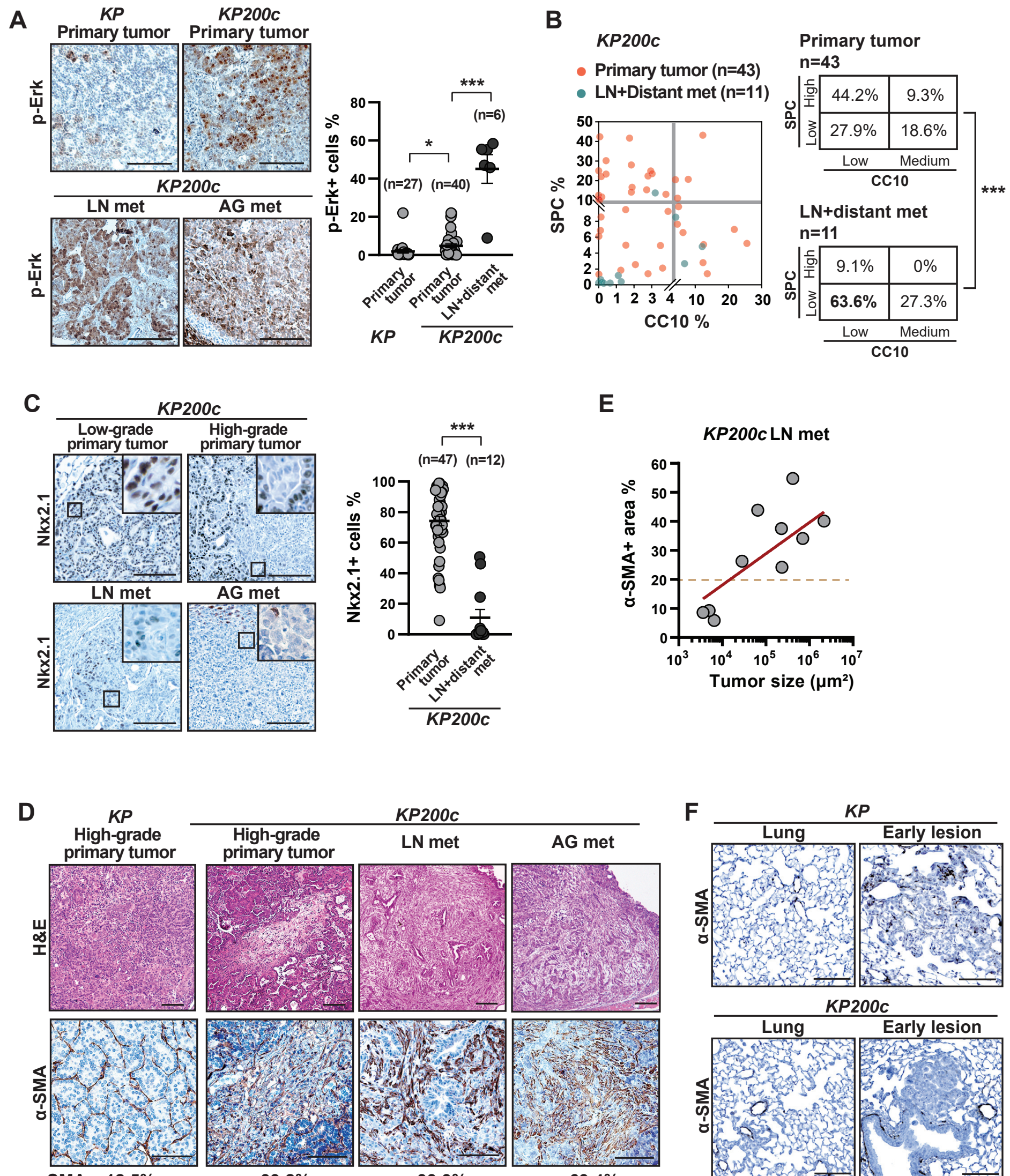

KP200c
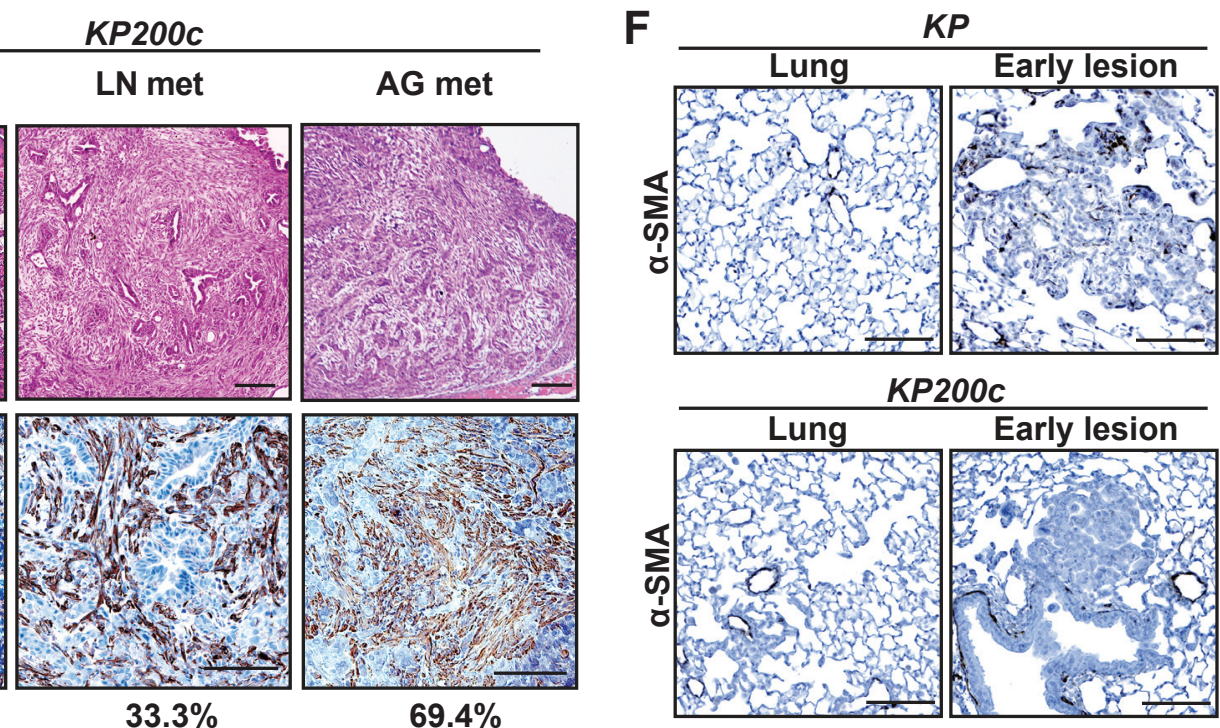


\section{Figure 2 cont'd}
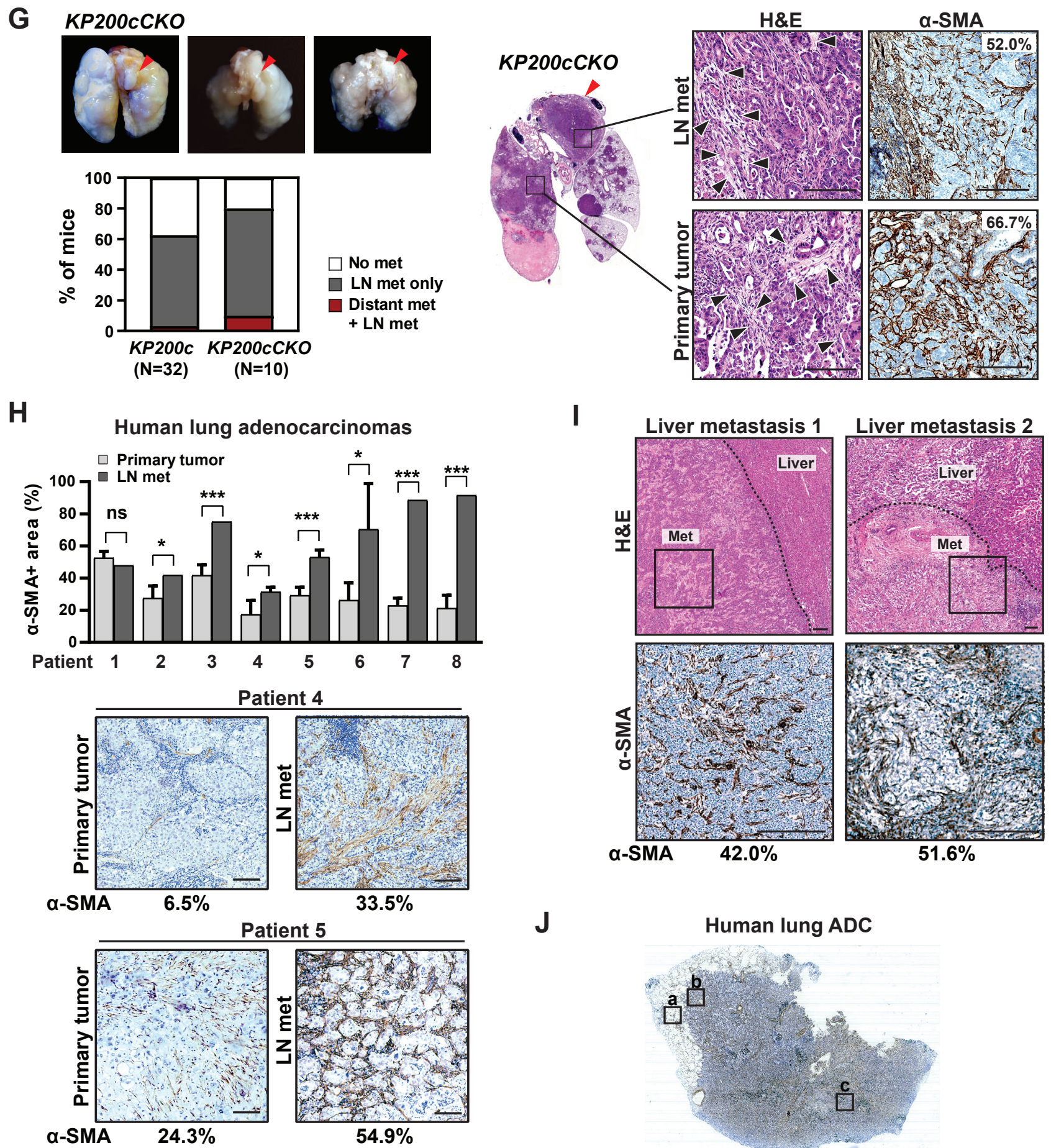

\section{Human lung ADC}
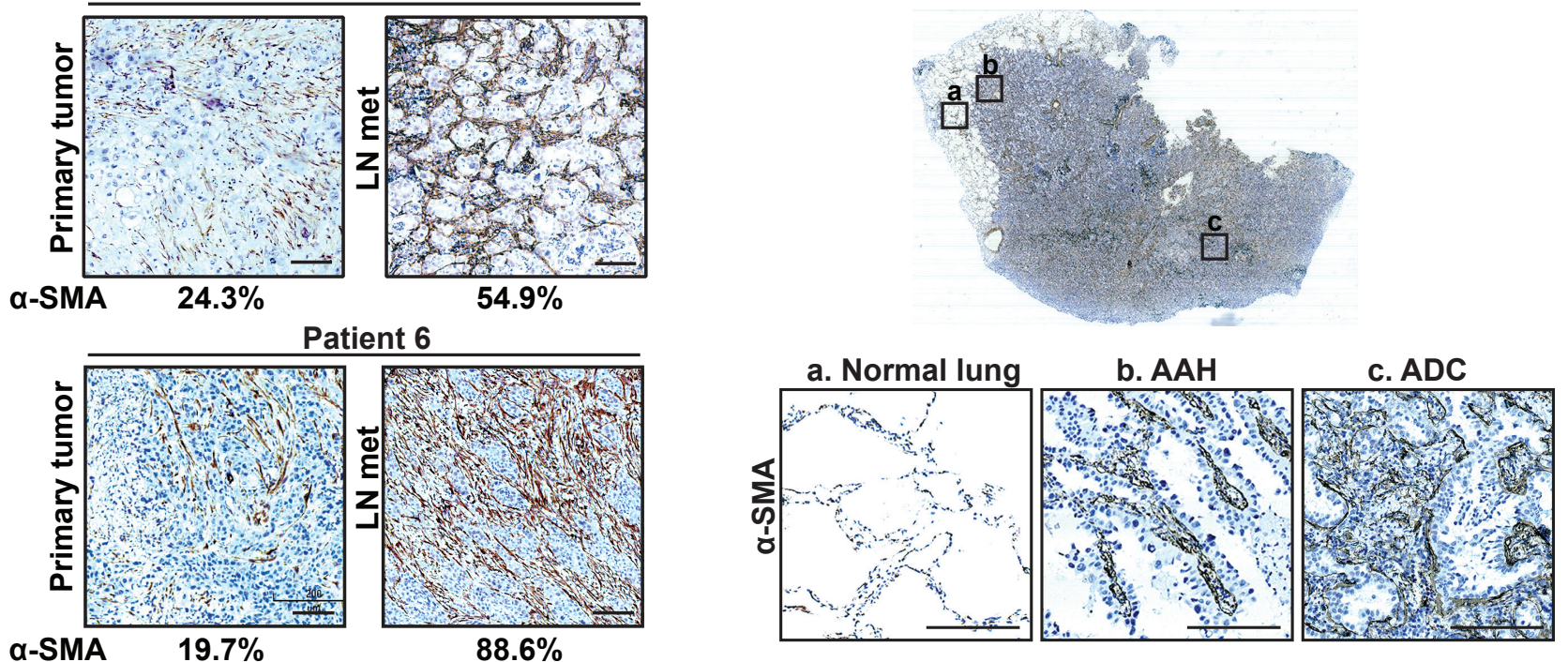


\section{Figure 3}

A
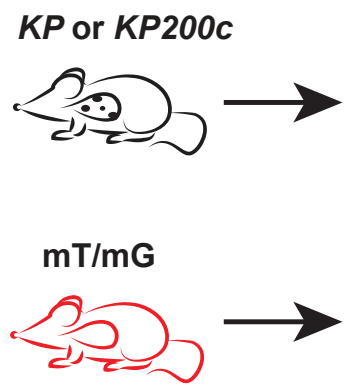

GFP labeled

Cancer cells

tdTomato+ Lung fibroblasts
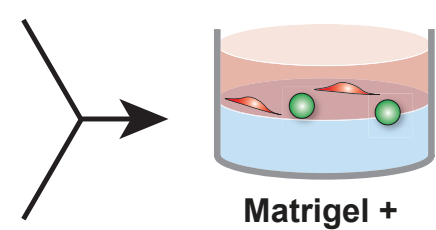

Matrigel + Collagen I

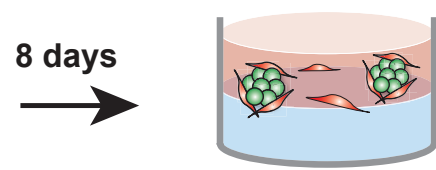

Tumor organoid

B
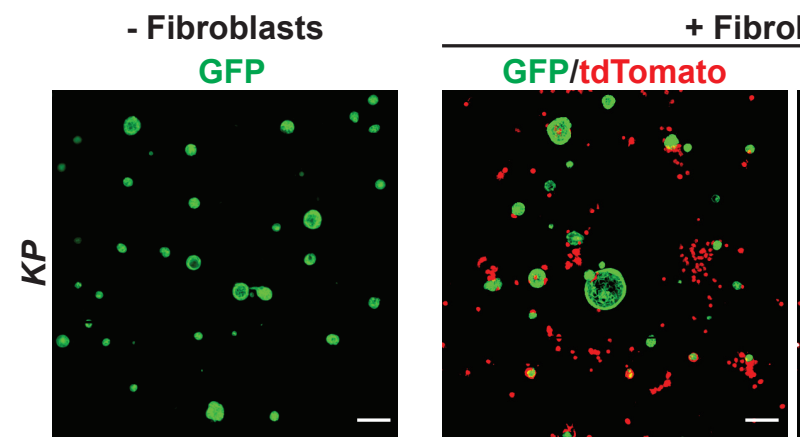

+ Fibroblasts
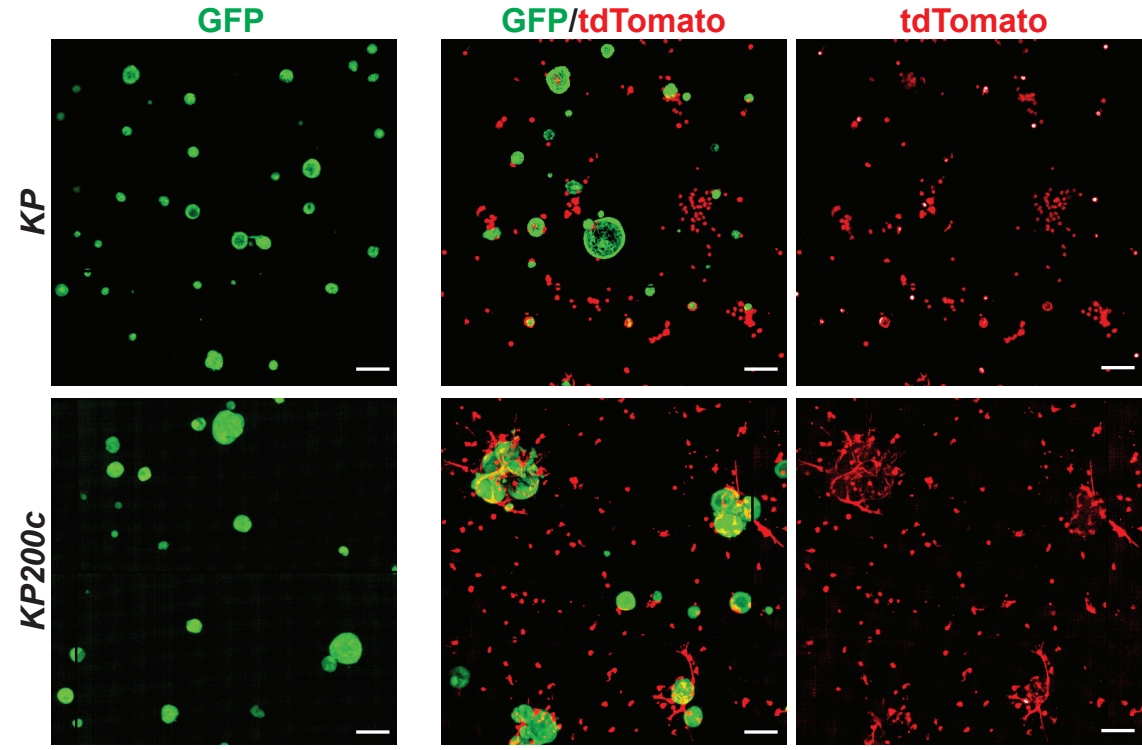

GFP: Cancer cells

tdTomato: Lung fibroblasts

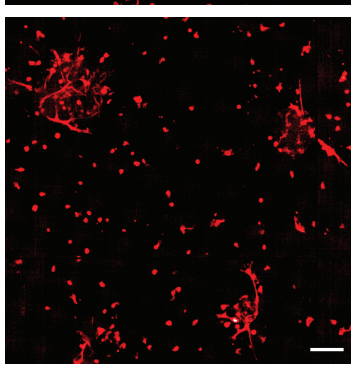

- $K P$ tumor organoid

- KP200c tumor organoid

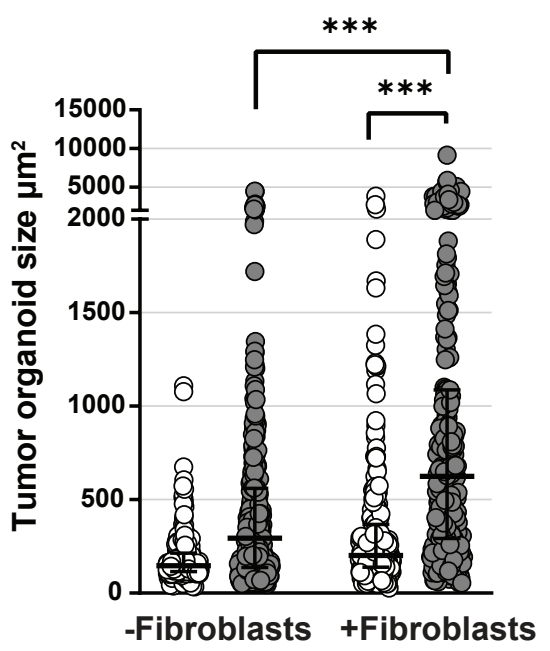

C

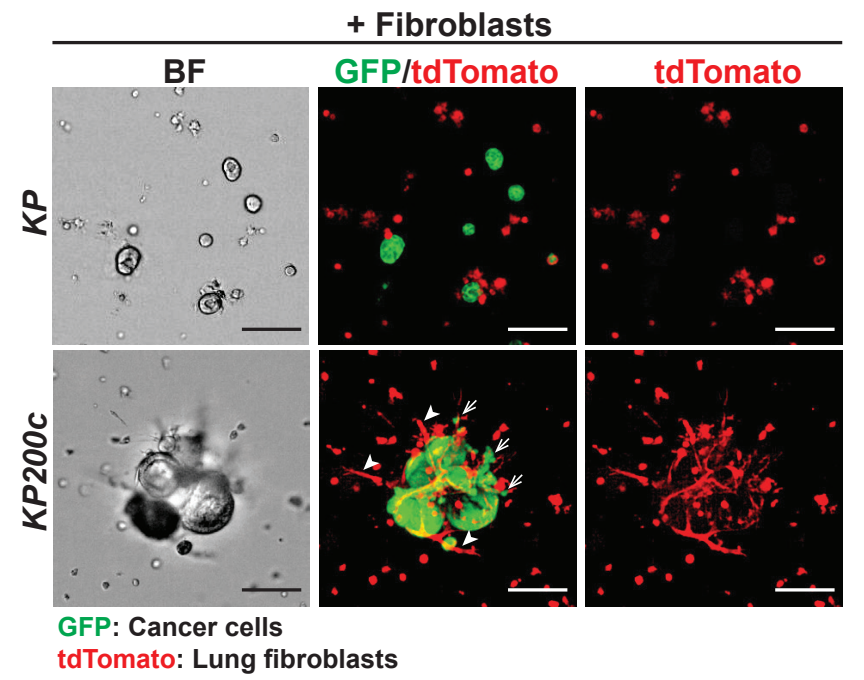

D
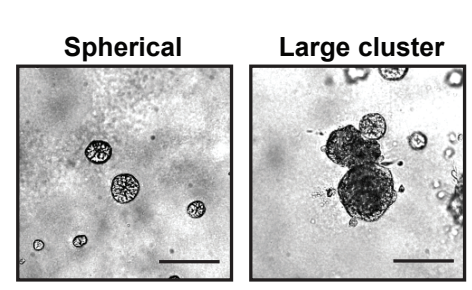

Cluster with invasive features
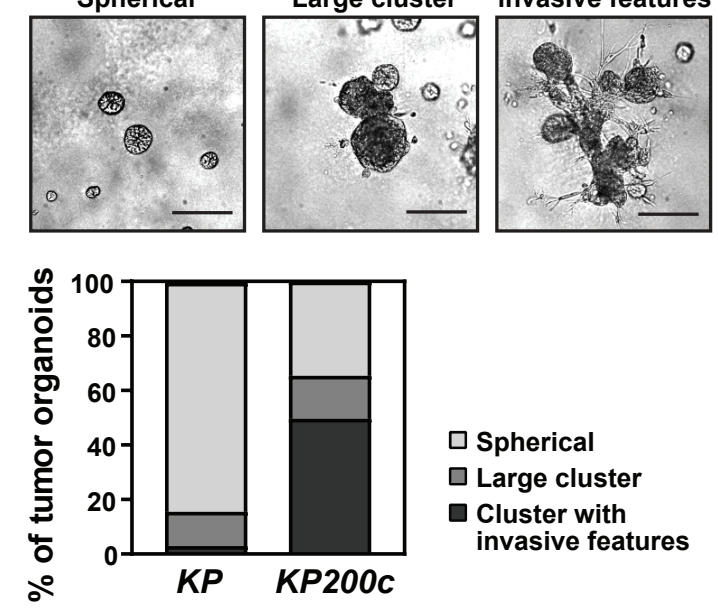

$\square$ Spherical

$\square$ Large cluster

- Cluster with invasive features 


\section{Figure 3 cont'd}

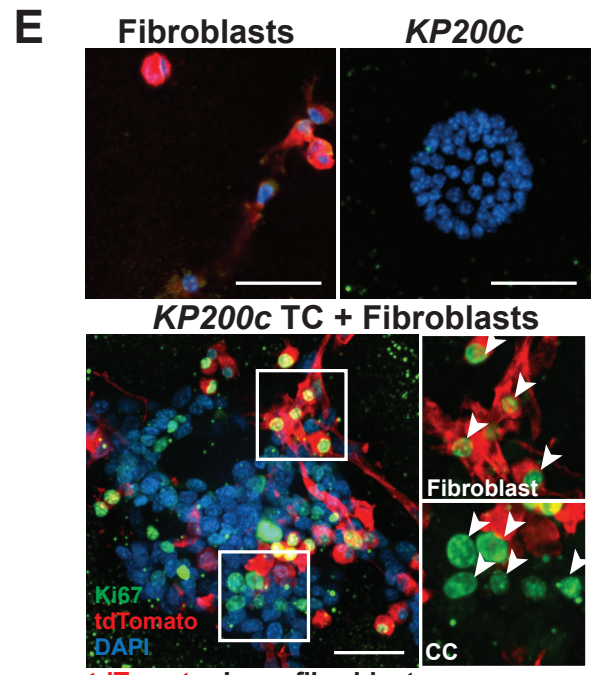

tdTomato: Lung fibroblasts

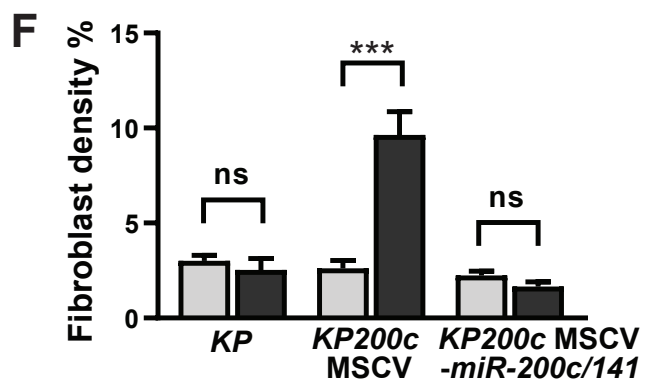

$\square$ Non-tumor associated fibroblasts - Tumor-associated fibroblasts

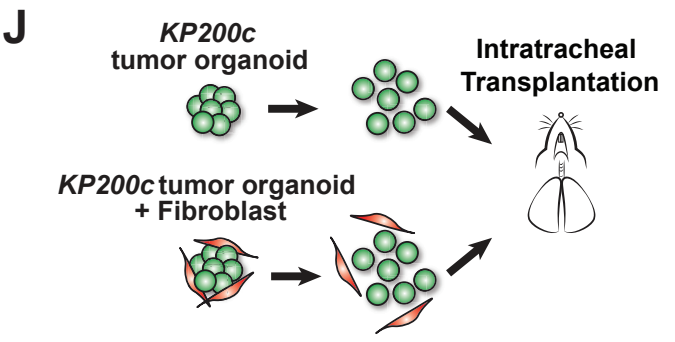

K
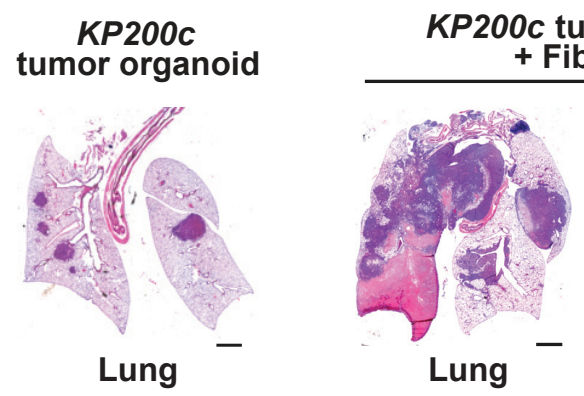

or organoid

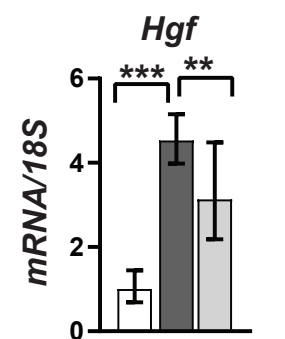

$\square$ Fibroblast only

$\square$ Fibroblasts w/ KP200c MSCV cancer cells

$\square$ Fibroblasts w/ KP200c MSCV-miR-200c/141 cancer cells
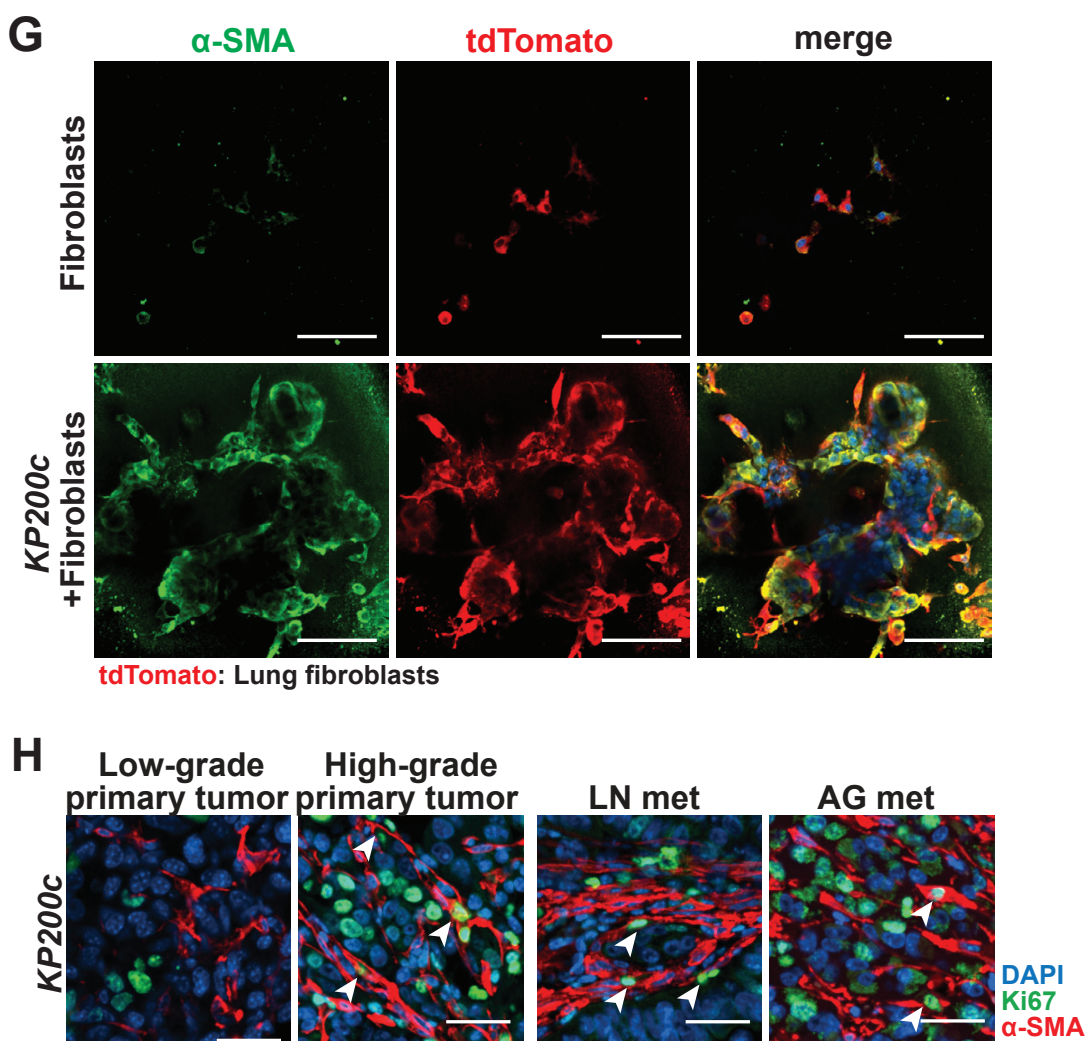

\section{Pleural lavage}

\section{○}




\section{Figure 4}

A

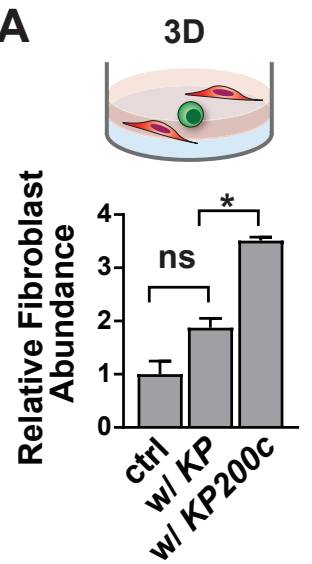

C

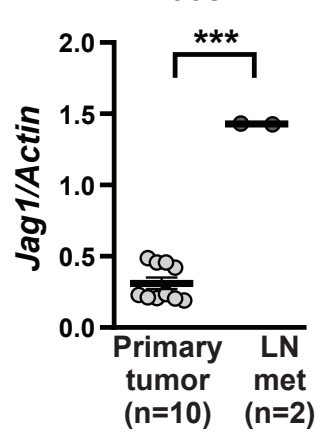

Transwell
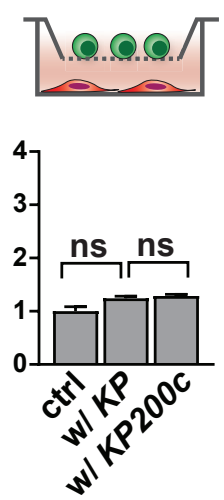

Jag2

Jag2 3'UTR (891-897)

miR-200c

miR-141
B Jag1

Jag1 3'UTR(1474-1480)

5' GGUGUUUGUUCUAU-UAGUGUUAU $3^{\prime}$ 3' GGÜAGAAAUGGUCUGUCACÁLU

5' CUCCCGGGACGGCGGCAGUAUUG $3^{\prime}$ || : |||||||||: $3^{\prime}$ AGUAGUAAUGGUCCGUCAUAAU 5'

E

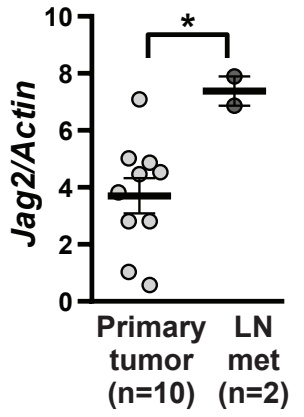

D KP200c-MSCV

$\square K P 200 c-M S C V-m i R-200 c / 141$
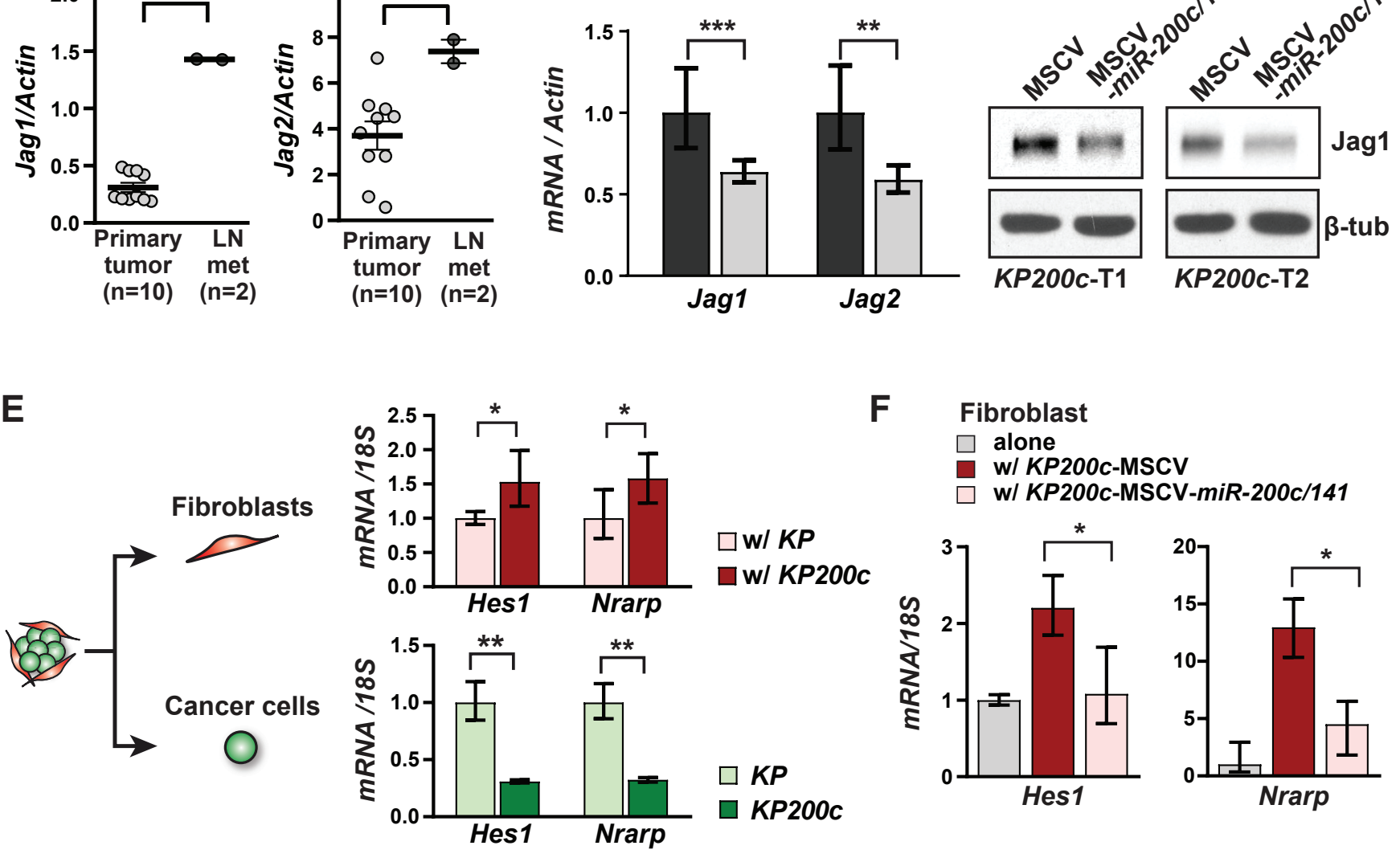

F Fibroblast

$\square$ alone

w/ KP200c-MSCV

w/ KP200c-MSCV-miR-200c/141
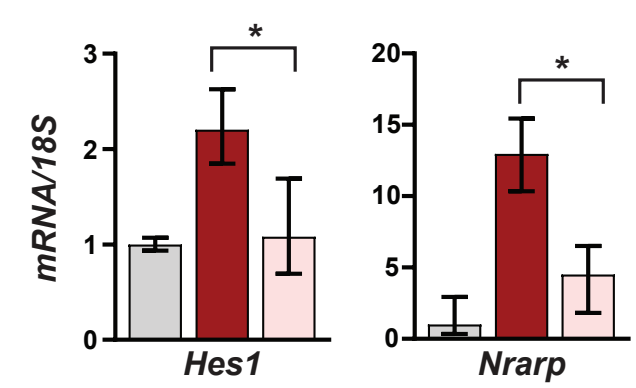

G

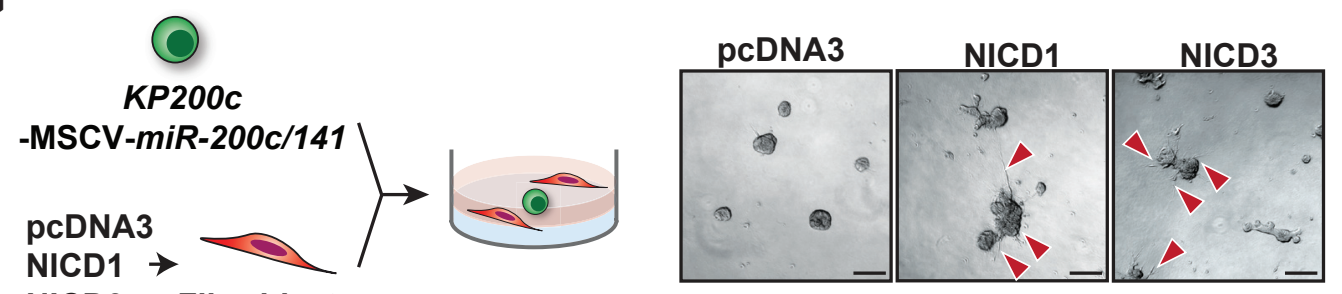

NICD3 Fibroblasts

$\square$ Spherical

$\square$ Large cluster

Cluster with invasive features

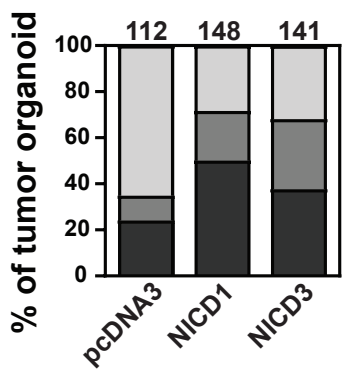




\section{Figure 4 cont'd}

H

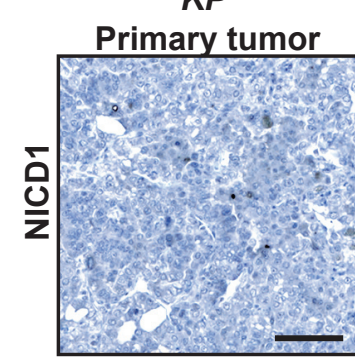

KP200c

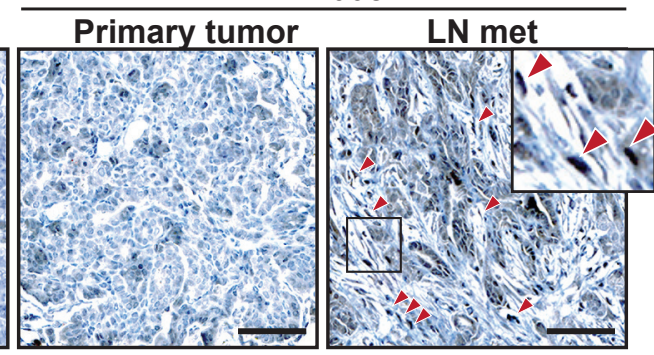

Patient 1

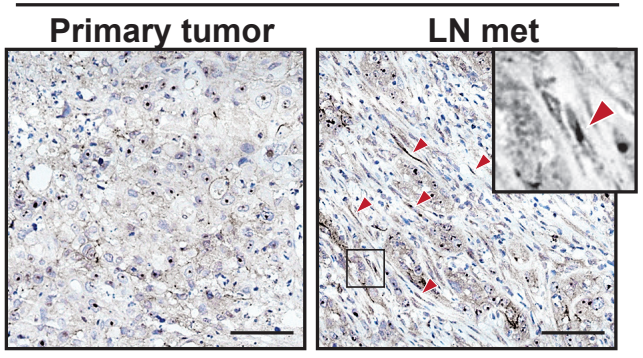

I
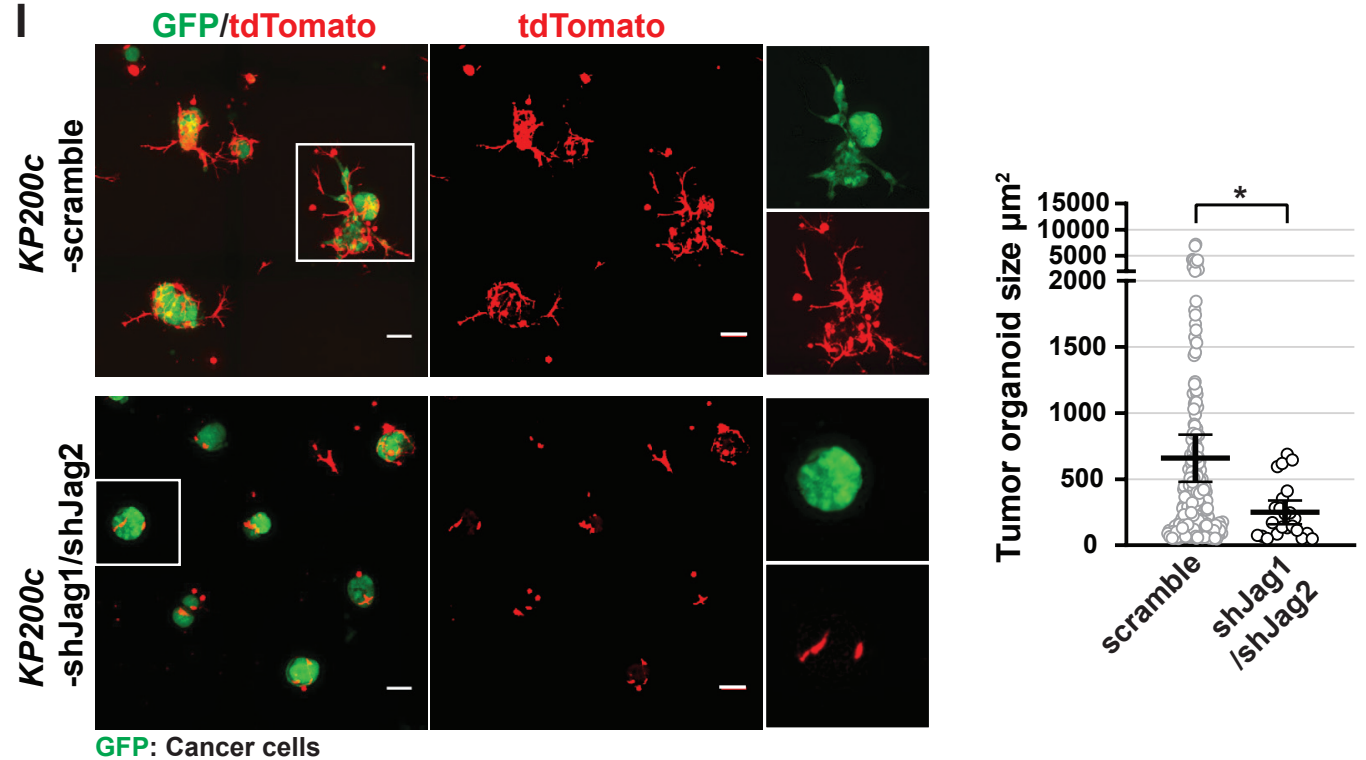

$\square$ Spherical

$\square$ Large cluster

Cluster with invasive features

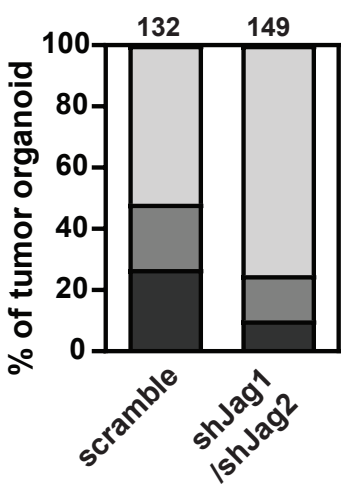

tdTomato: Lung fibroblasts

$\mathbf{J}$ miR-200-expressing tumor

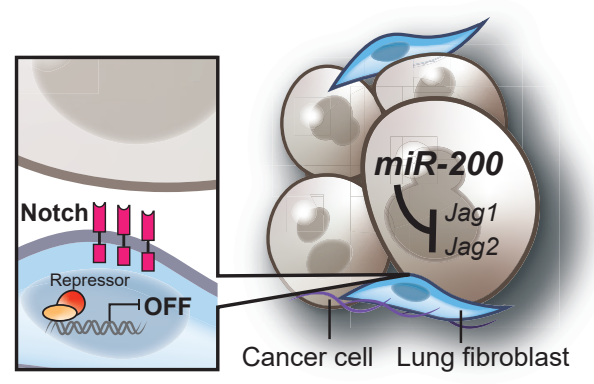

miR-200-deficient tumor

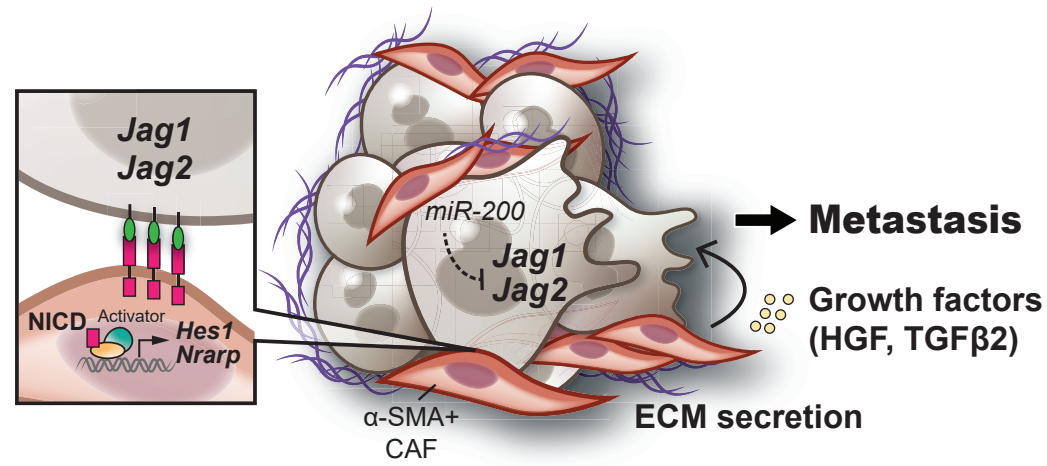

Fibroblast

- Proliferation

- Induction of a-SMA

- Secretion of growth factors \& ECM

\section{Cancer cell}

- Proliferation

- Promote invasiveness

- Promotes metastatic potential 


\section{Supplementary Figure S1}

A

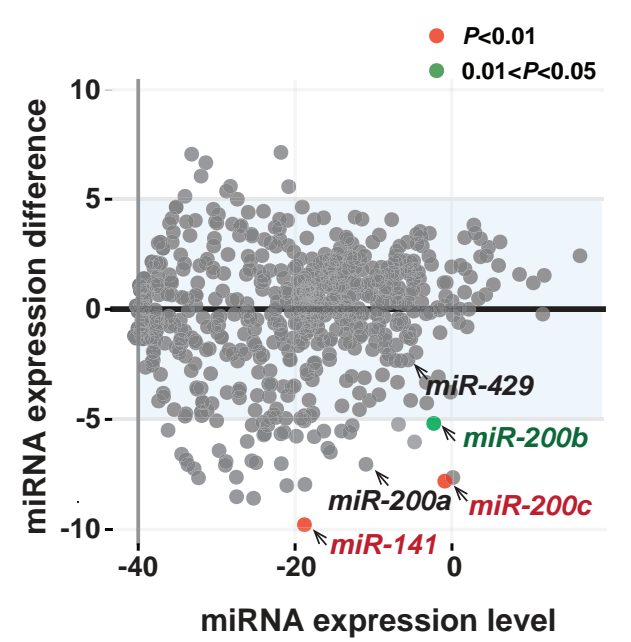

C

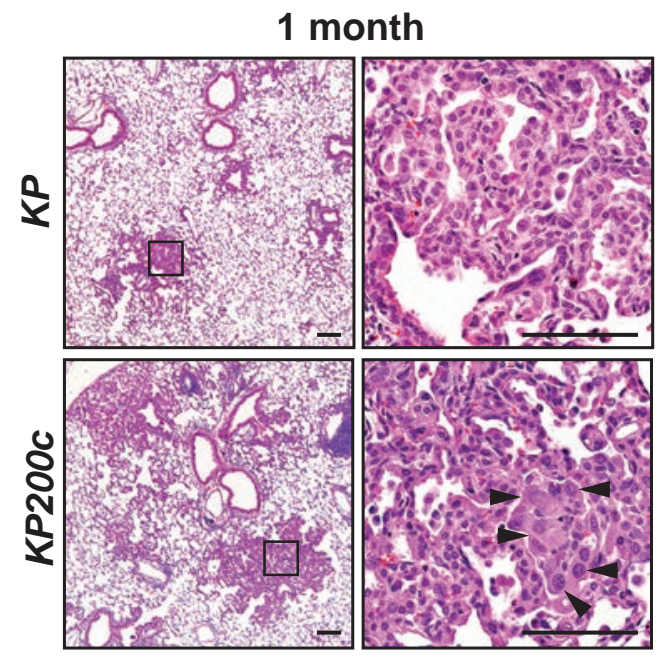

B mir-200c/141

mir-200c/141

targeting vector

mir-200c/141 ${ }^{\text {puro- }-\Delta t k}$

' Arm 5.5kb

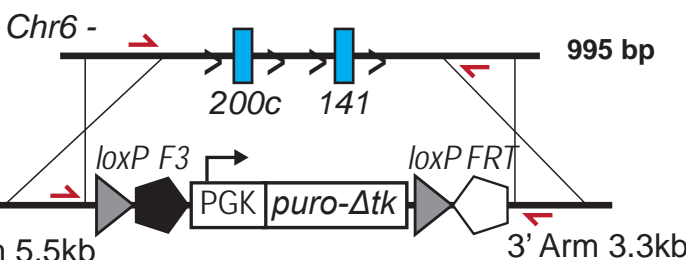

mir-200c/141 null

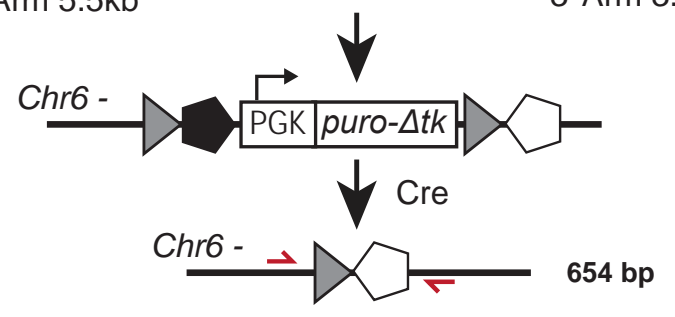

$\operatorname{mir}-200 c / 141$

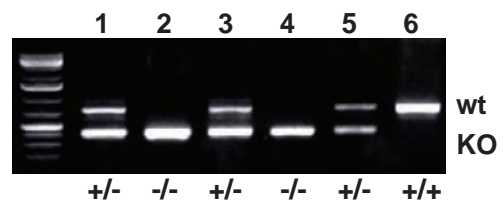

D miR-200c
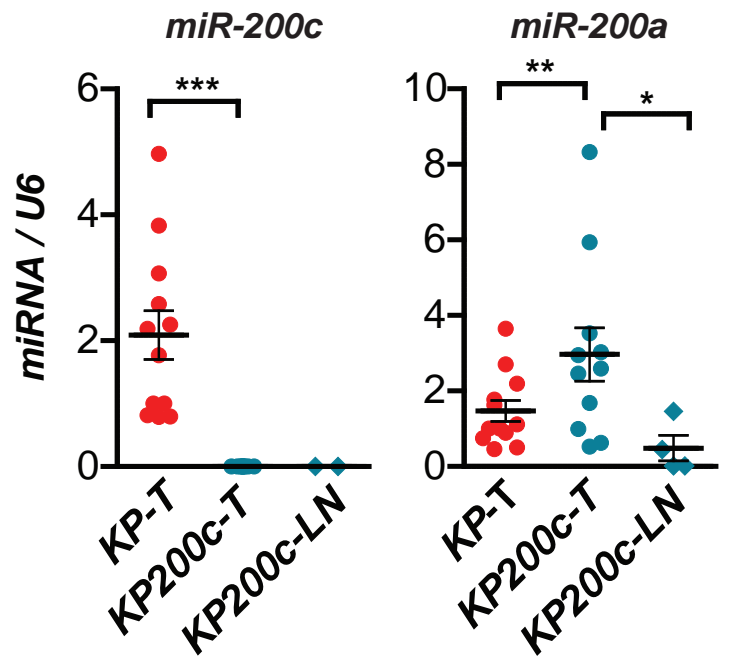

E
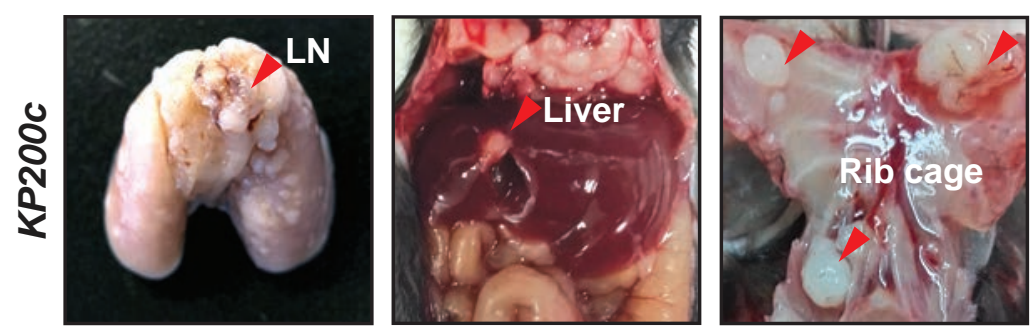
bioRxiv preprint doi: https://doi.org/10.1101/2020.09.02.276550; this version posted September 3, 2020. The copyright holder for this preprint (which was not certified by peer review) is the author/funder. All rights reserved. No reuse allowed without permission.

\section{Supplementary Figure S2}

A
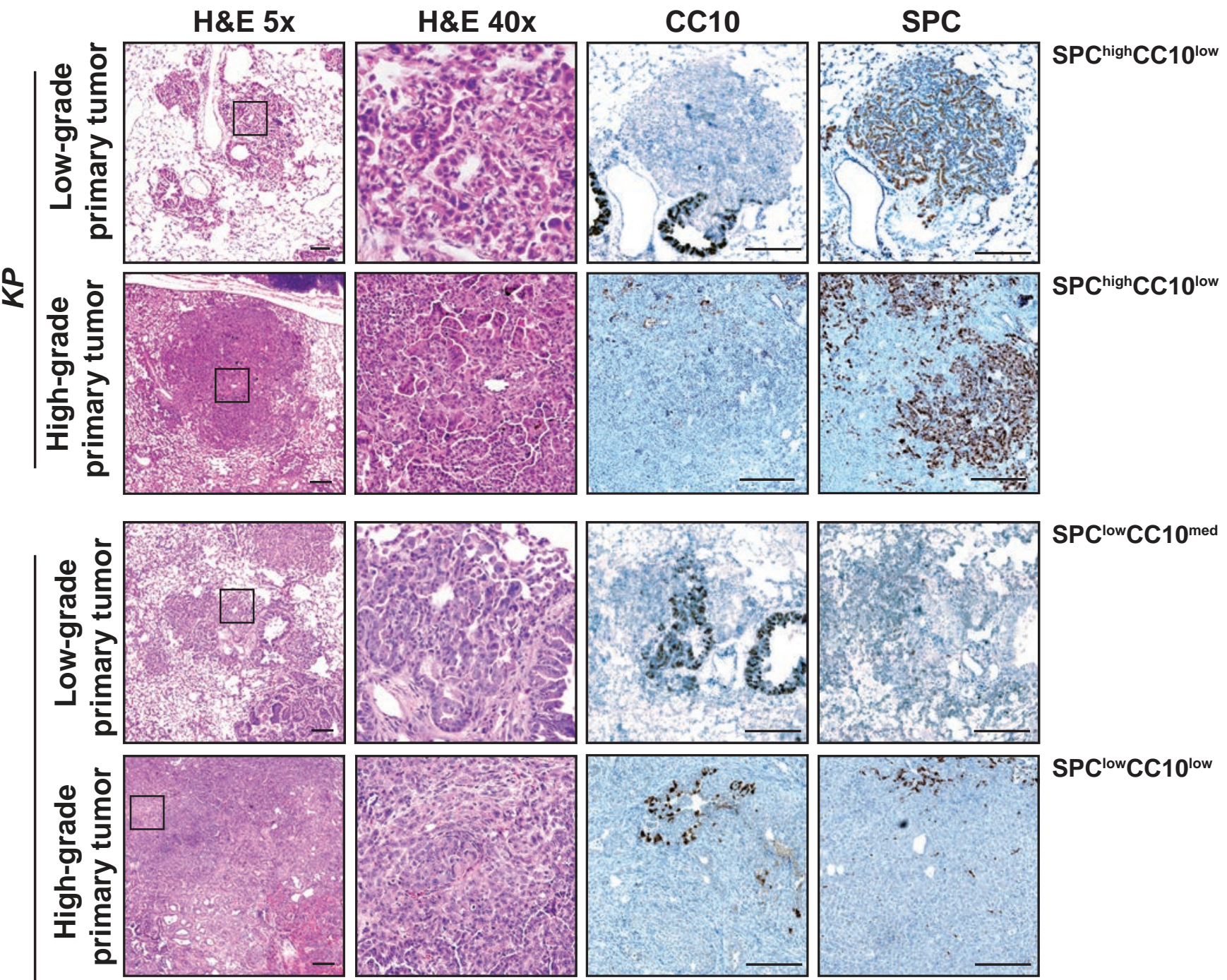

SPC ${ }^{\text {high }} \mathrm{CC} 10^{\text {low }}$
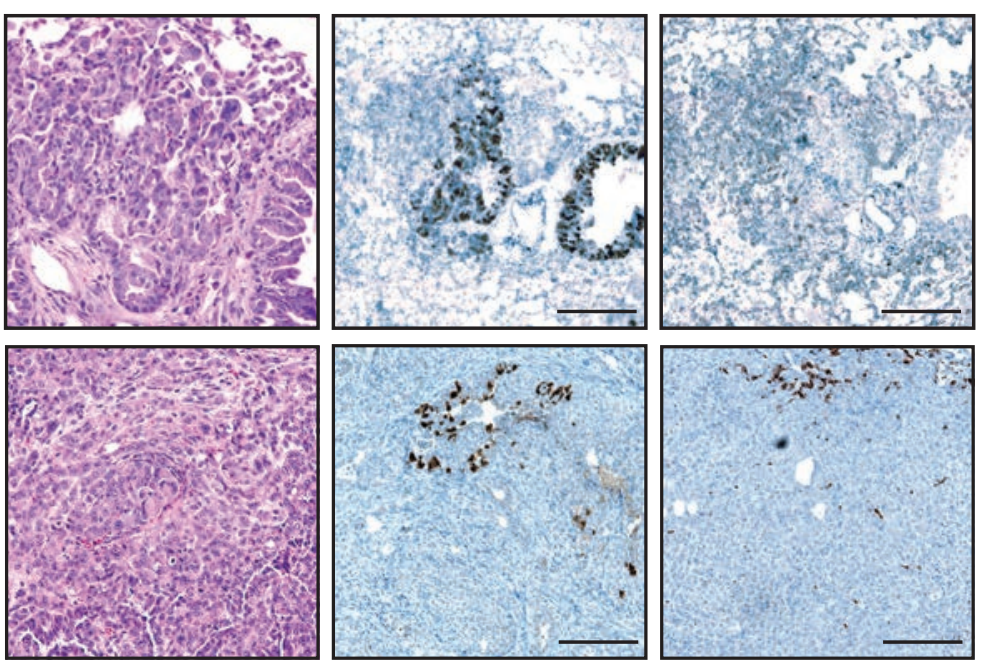

$\mathrm{SPC}^{\text {low }} \mathrm{CC} 10^{\mathrm{med}}$

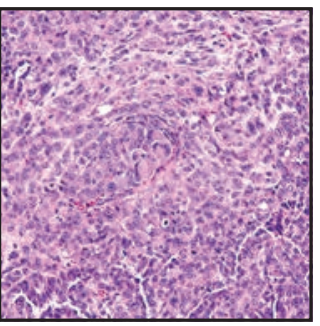

SPC ${ }^{\text {low }} C \mathrm{C} 10^{\text {low }}$
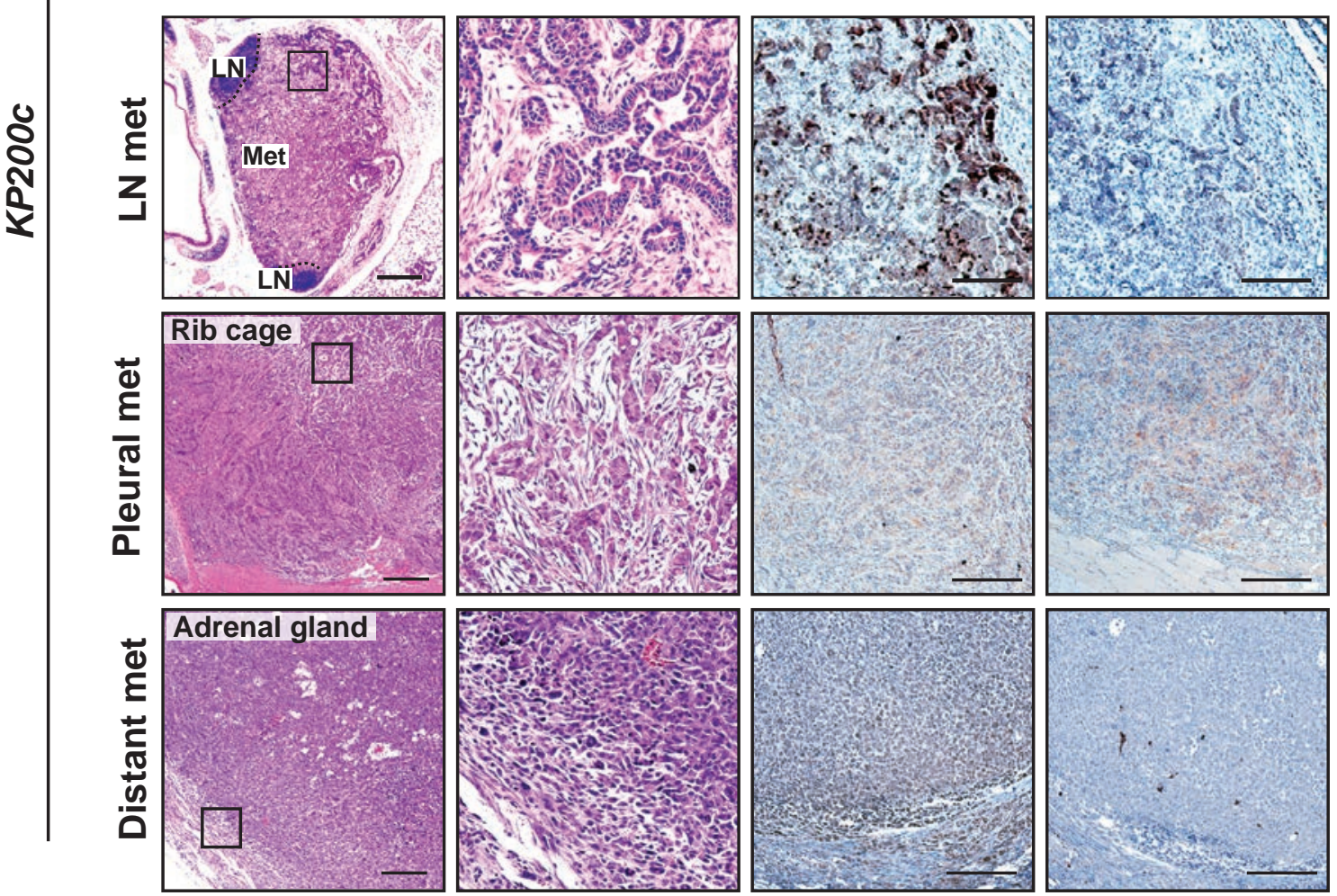
bioRxiv preprint doi: https://doi.org/10.1101/2020.09.02.276550; this version posted September 3, 2020. The copyright holder for this preprint (which was not certified by peer review) is the author/funder. All rights reserved. No reuse allowed without permission.

\section{Supplementary Figure S2 cont'd}

B

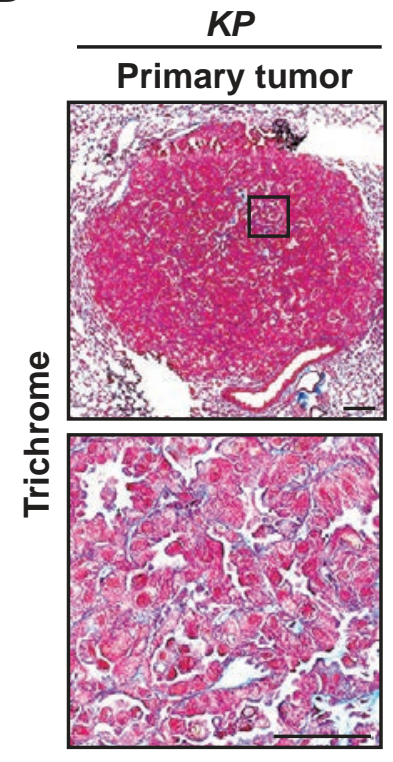

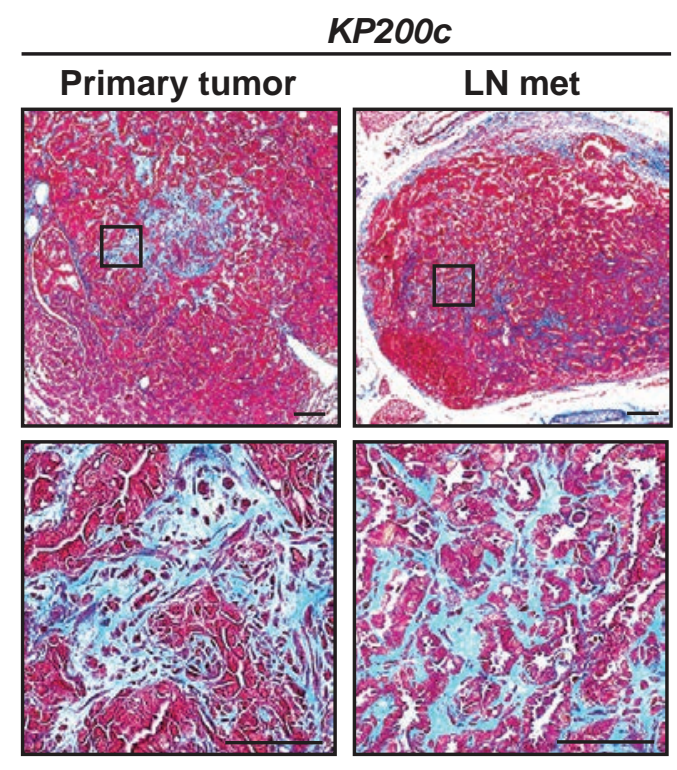

C

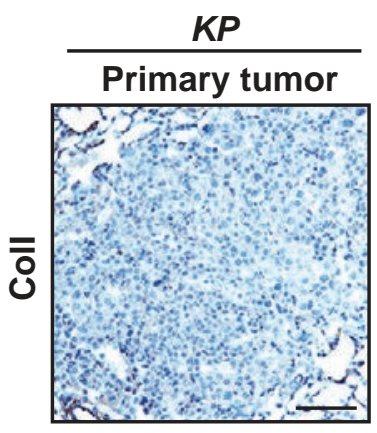

\section{KP200c}

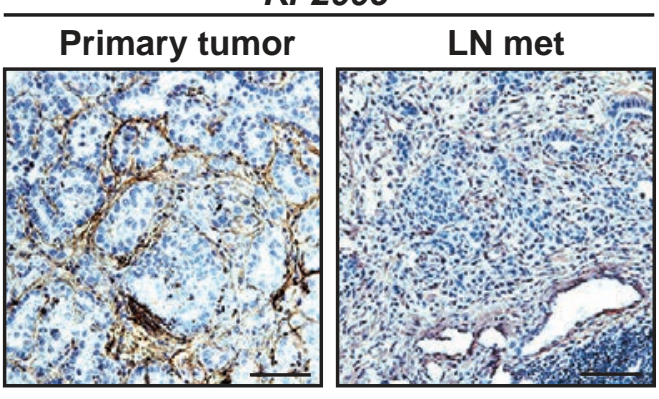

\section{Normal LN}

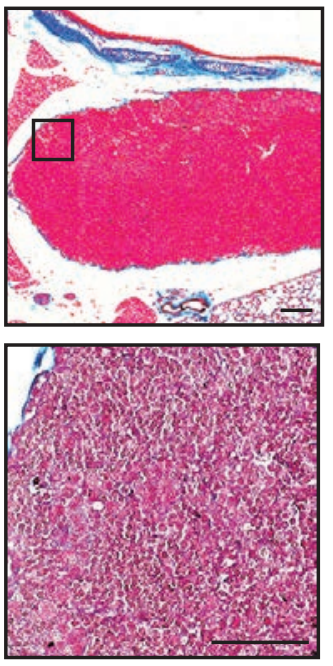

D

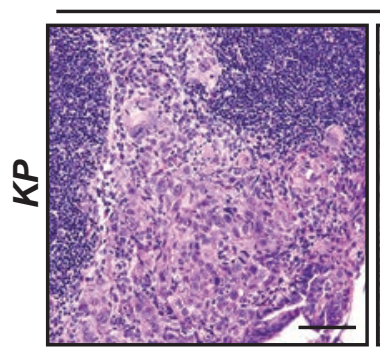

LN met
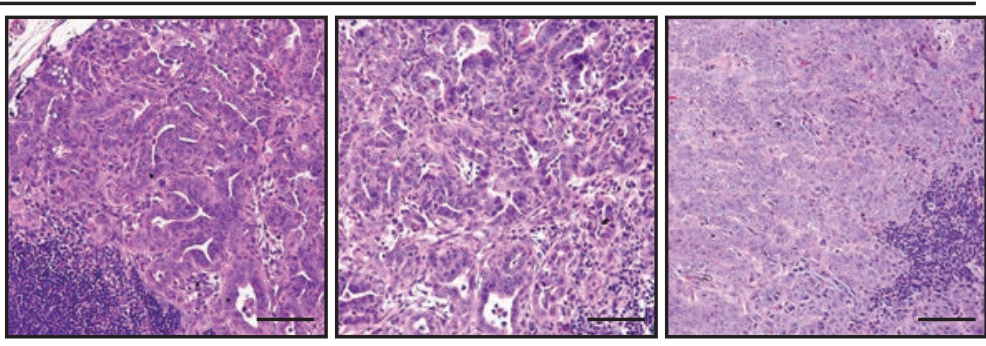

Liver met

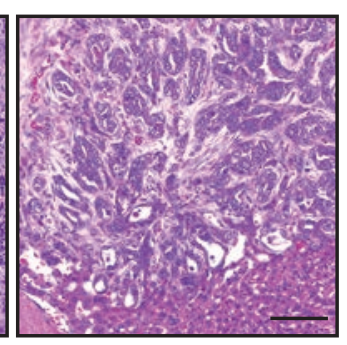

LN met
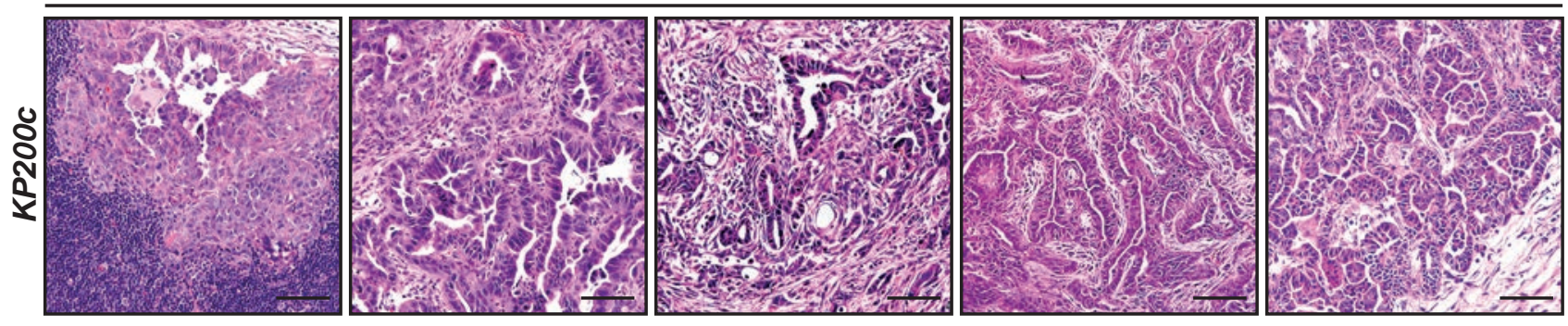
bioRxiv preprint doi: https://doi.org/10.1101/2020.09.02.276550; this version posted September 3, 2020. The copyright holder for this preprint (which was not certified by peer review) is the author/funder. All rights reserved. No reuse allowed without permission.

\section{Supplementary Figure S2 cont'd}

E

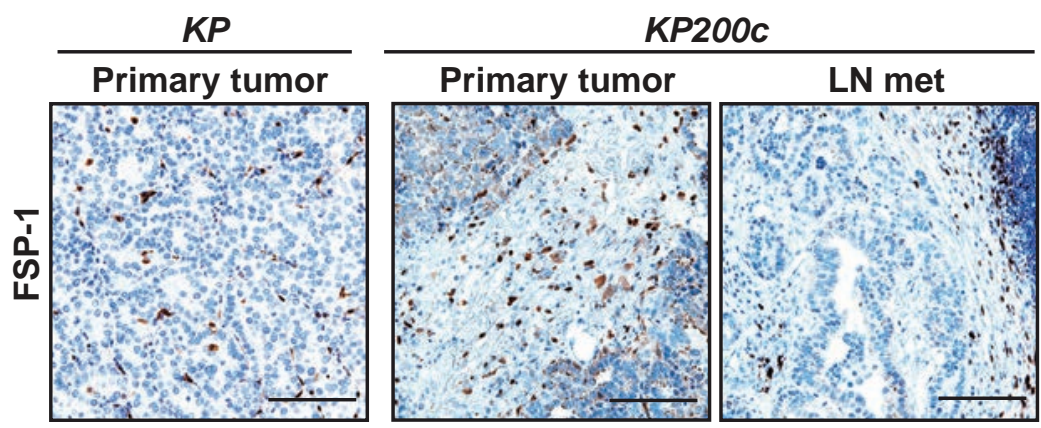

$\mathbf{F}$
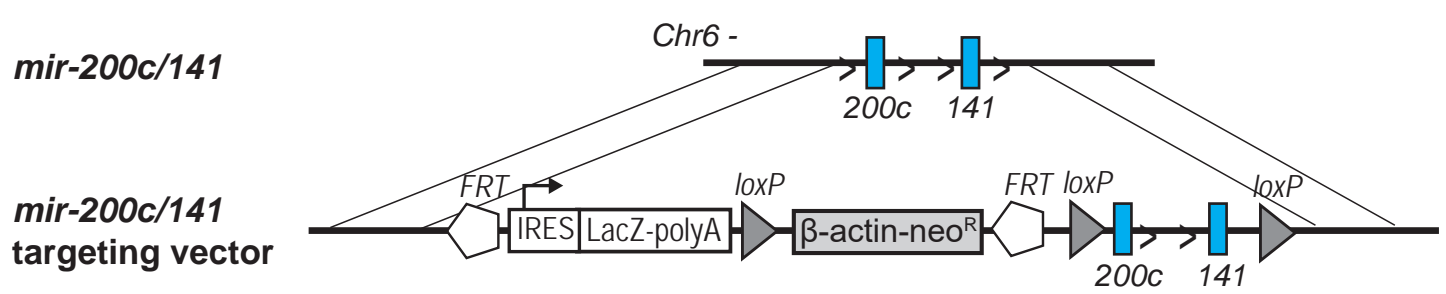

mir-200c/141 1 lacz

Chr6 -

4 Recombination

IOXP

FRT IOXP

$\beta$-actin-neo ${ }^{\mathrm{R}}$

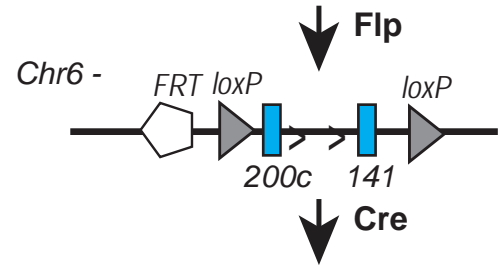

mir-200c/141 flox null

Chr6 - FRT IOXP

G

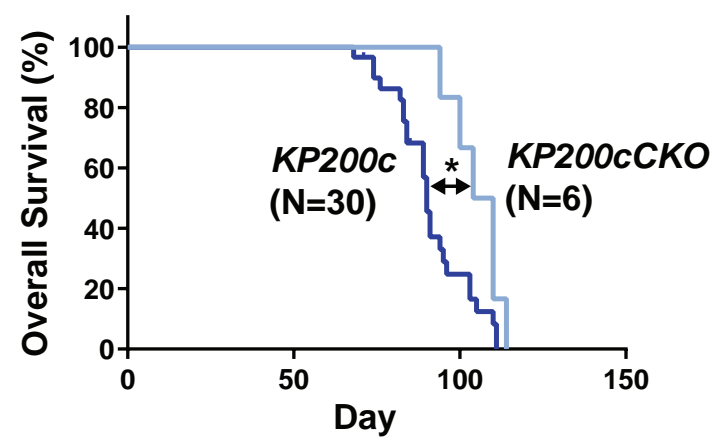




\section{Supplementary Figure S3}

A

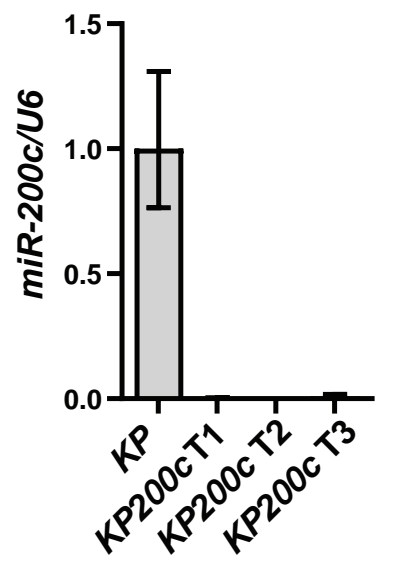

B

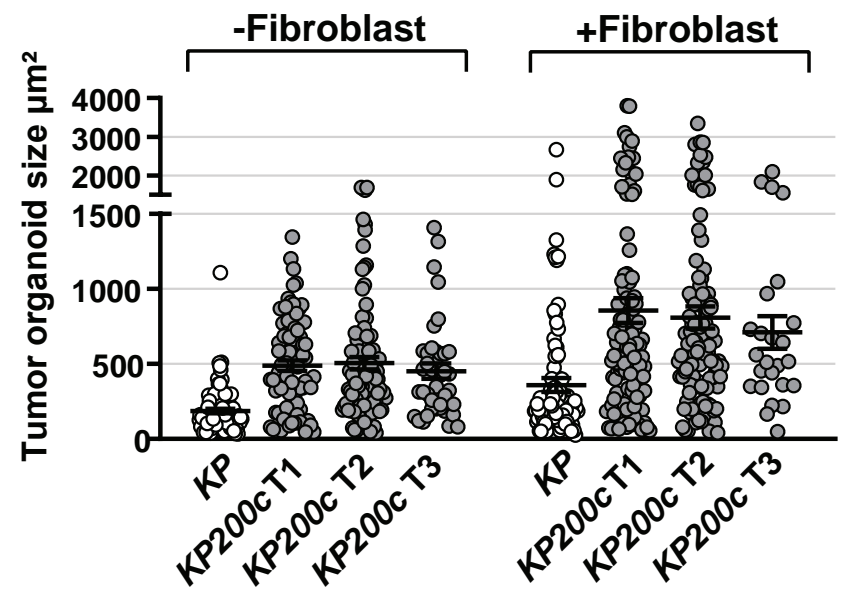

C KP200c cancer cell : Fibroblast
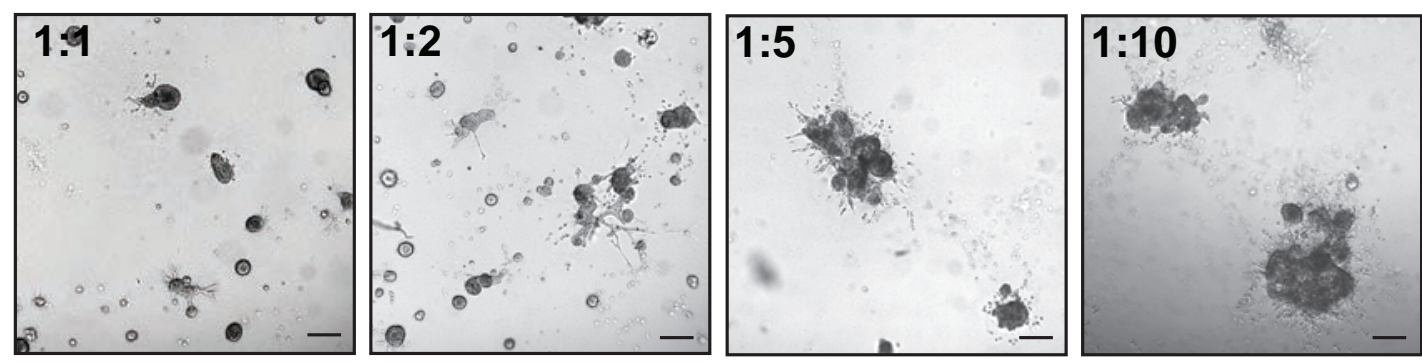

D

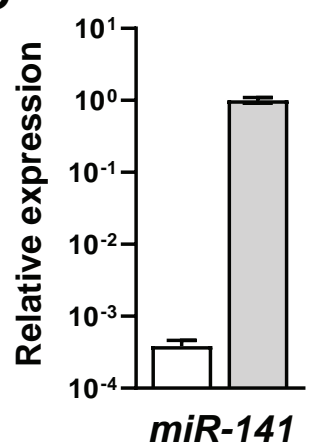

E

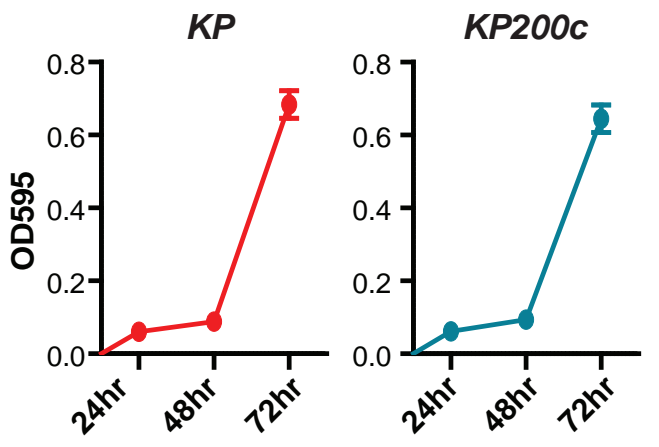

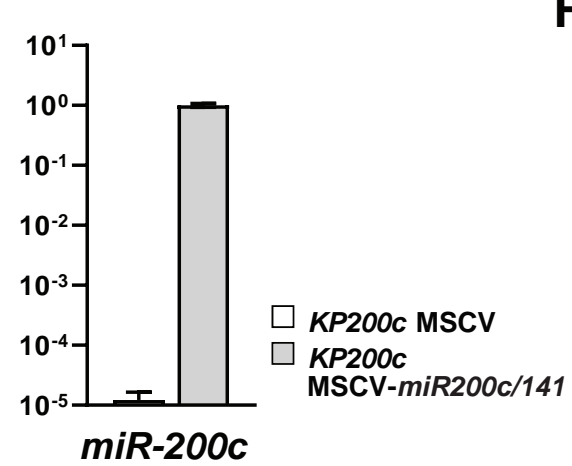

F KP200c MSCV

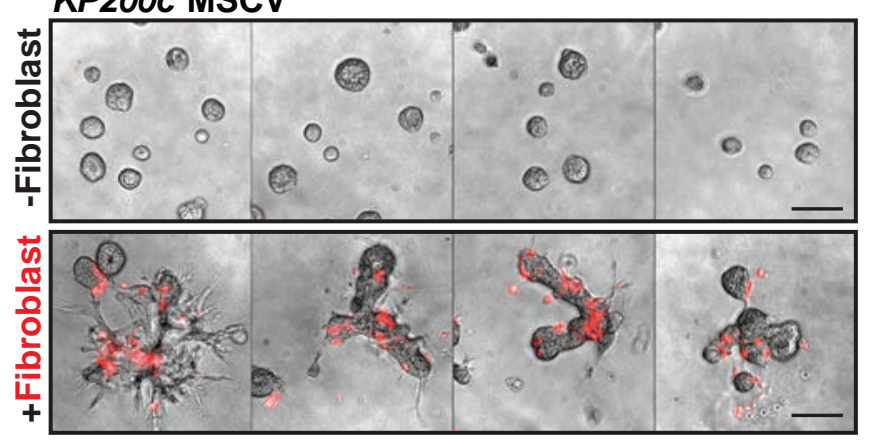

KP200c MSCV-miR200c/141

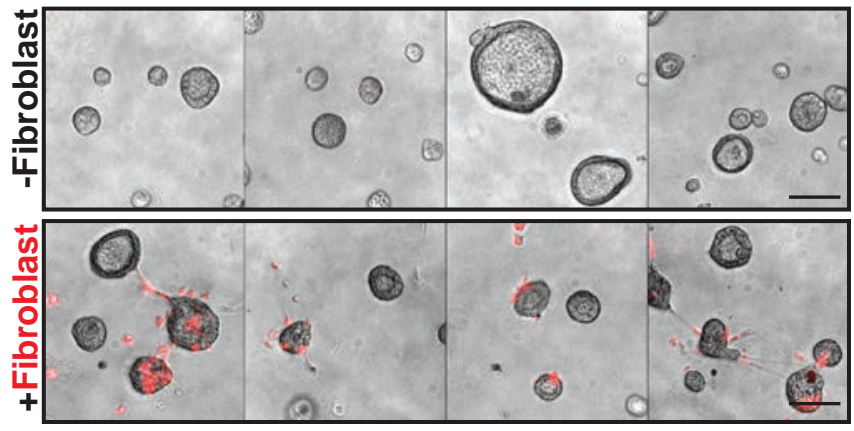




\section{Supplementary Figure S3 cont'd}

G
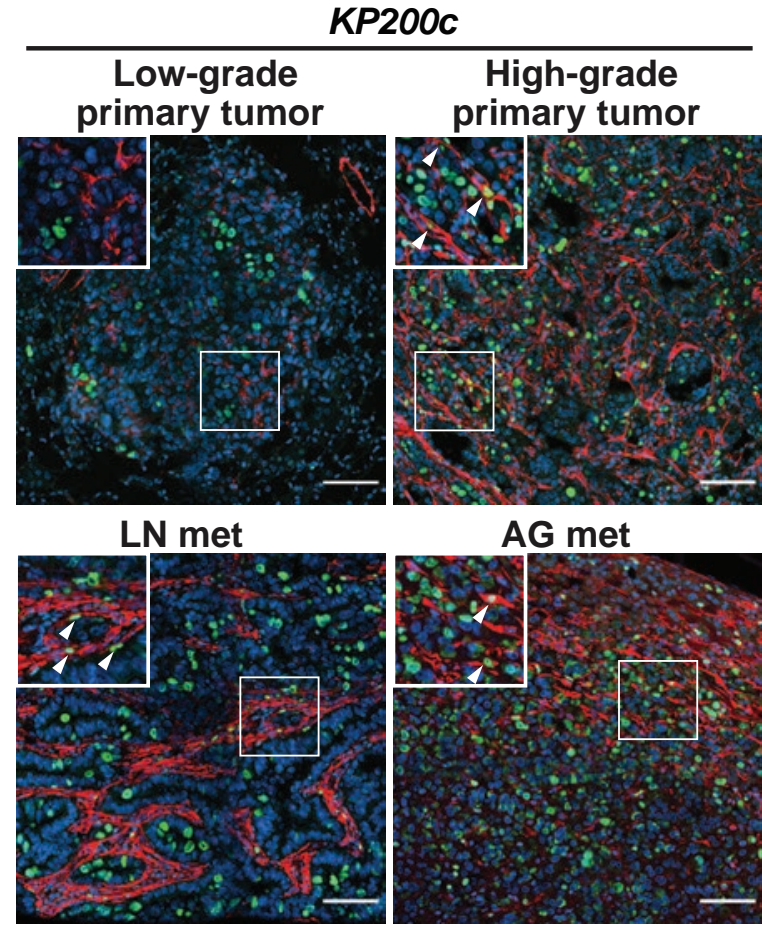

High-grade primary tumor

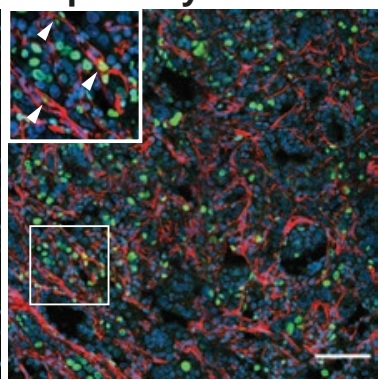

AG met
H
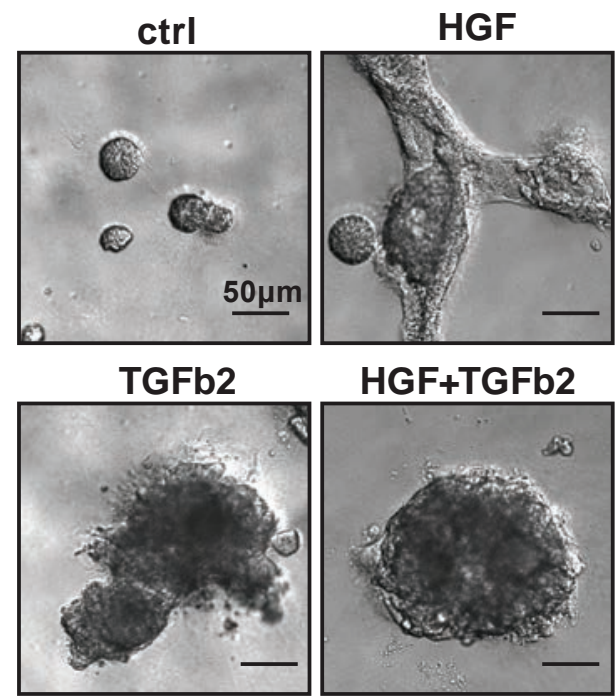

HGF+TGFb2

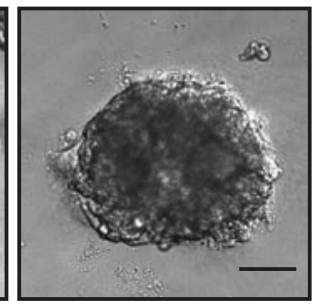

$\mathbf{L}$

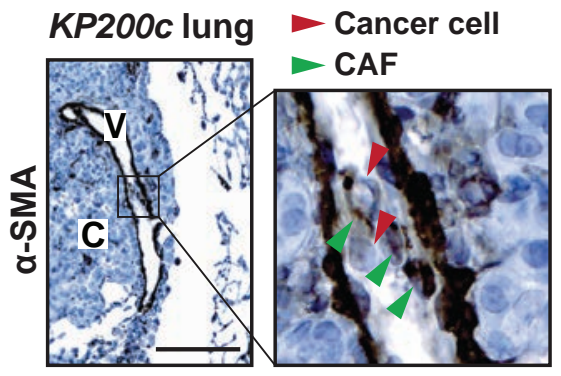

K

M

$\mathbf{N}$
I
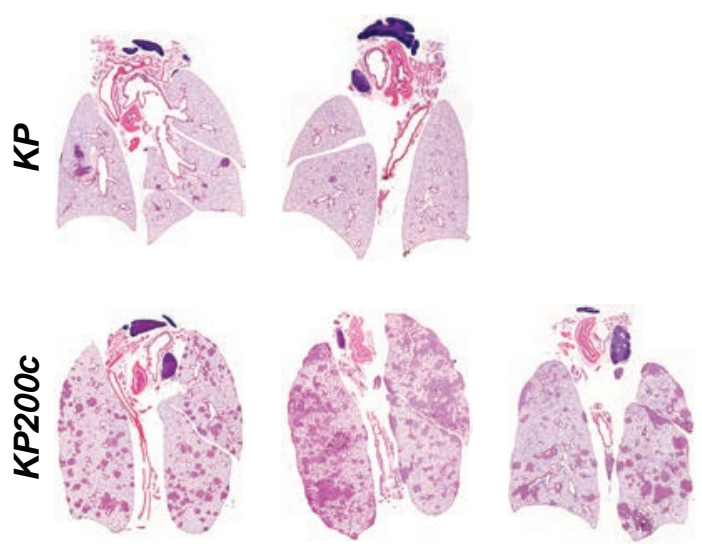

J
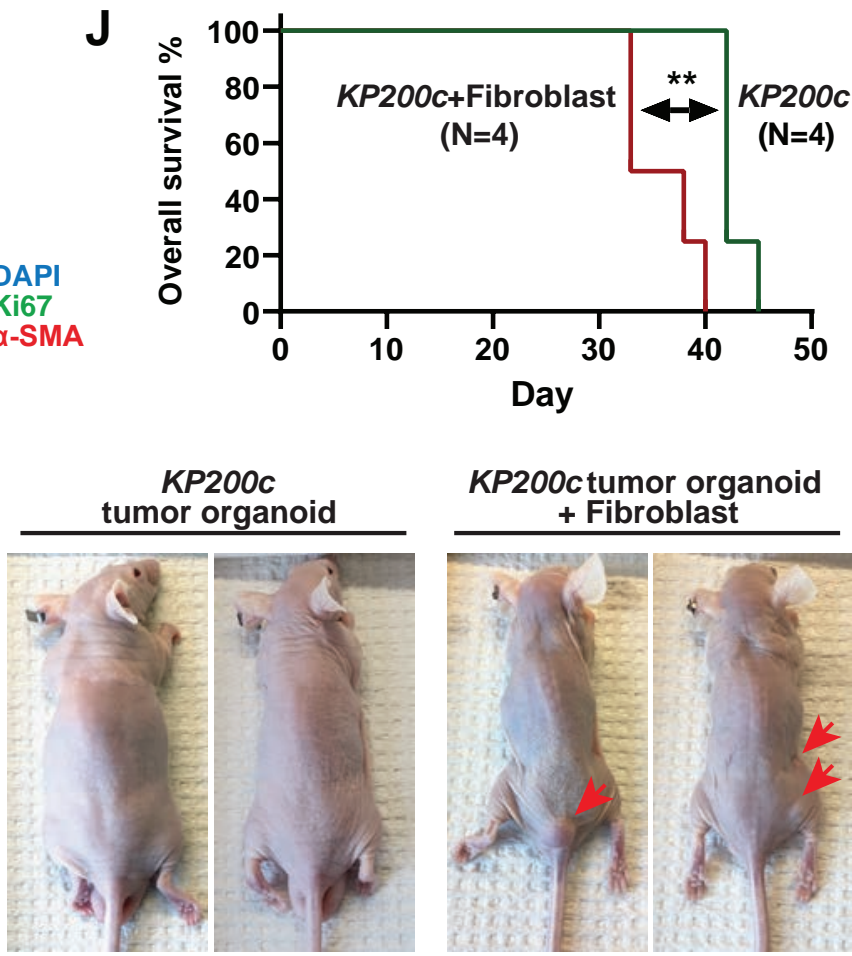

KP200c tumor organoid
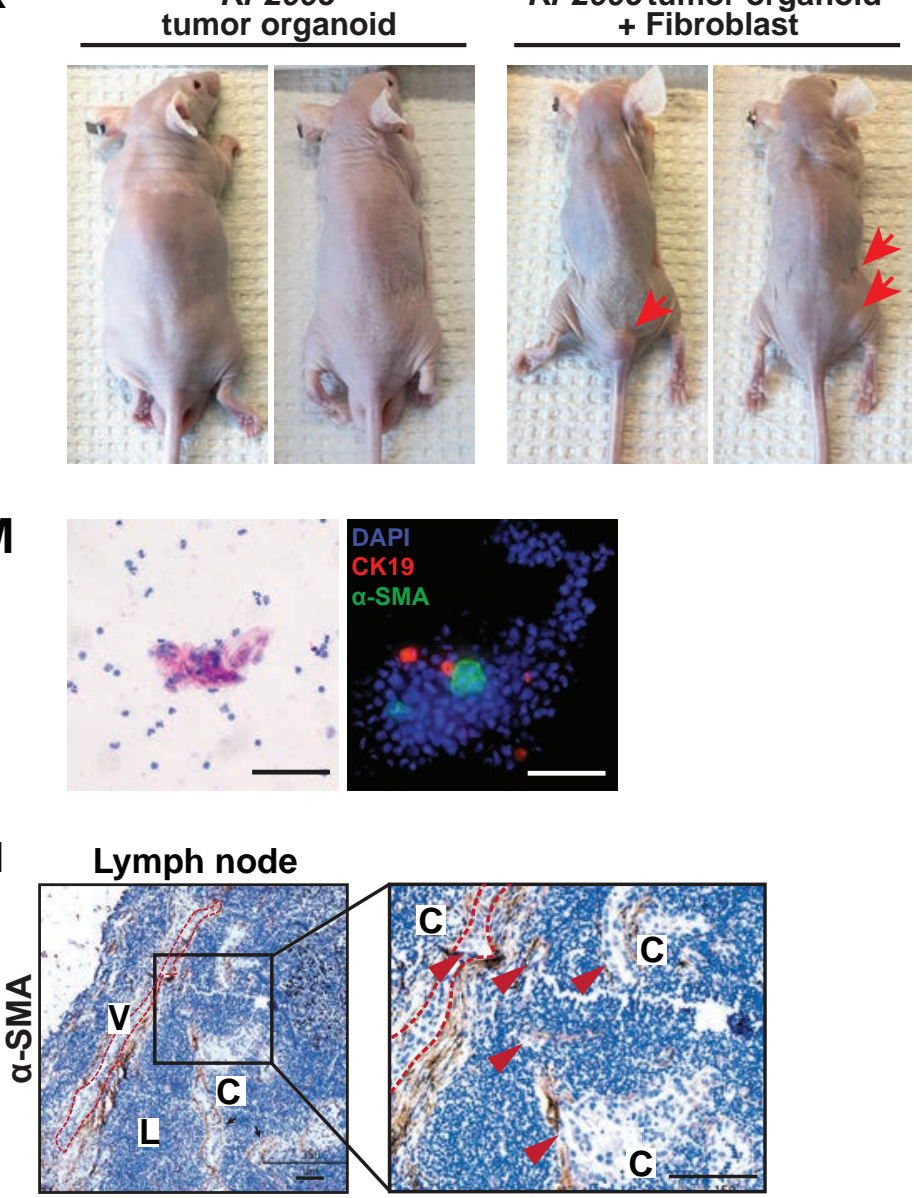


\section{Supplementary Figure S4}

A
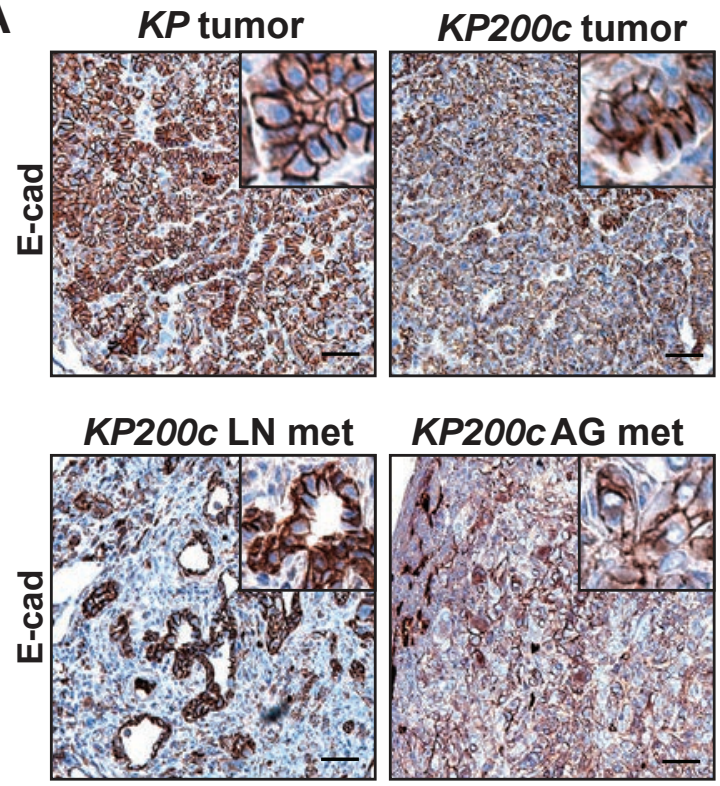

C

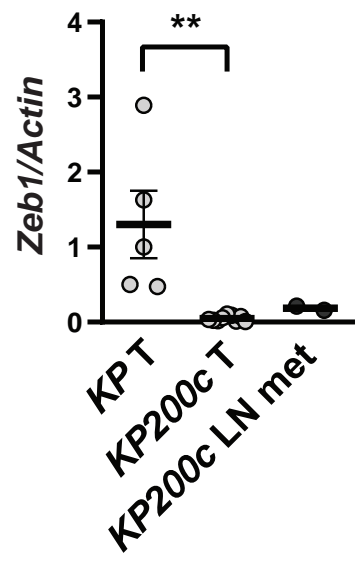

F

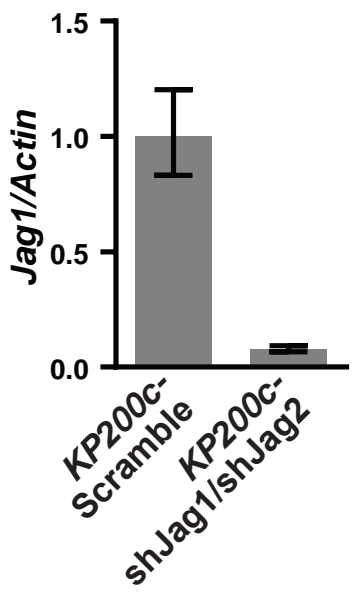

KP200c AG met
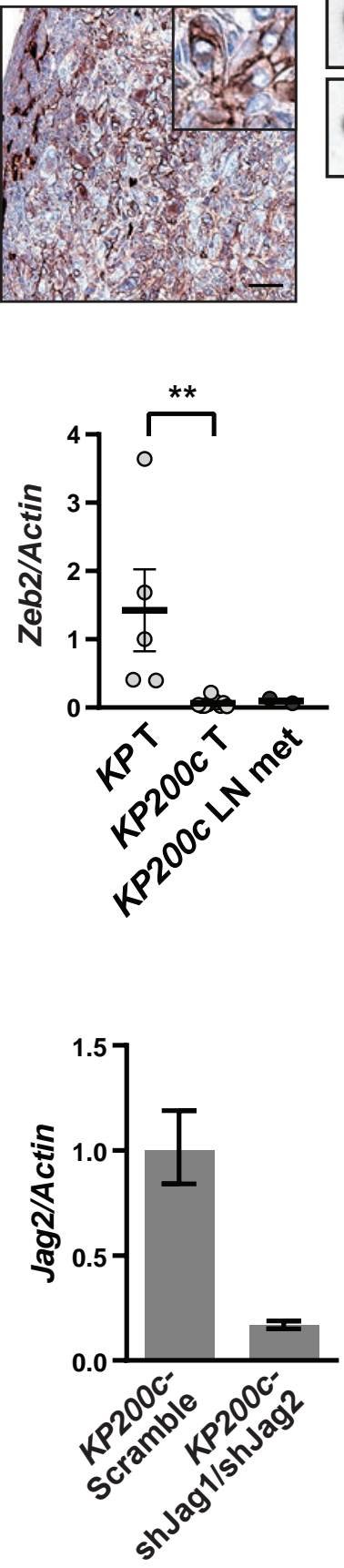
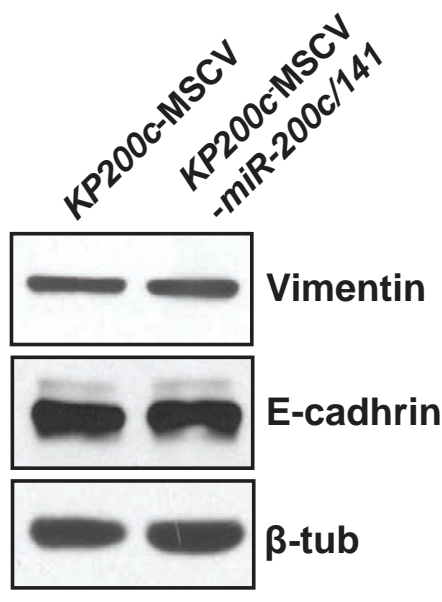

B

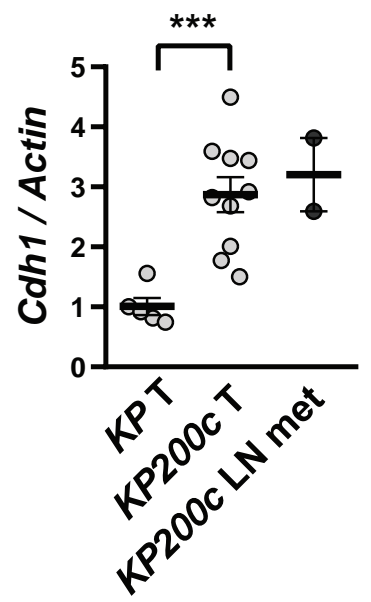

D
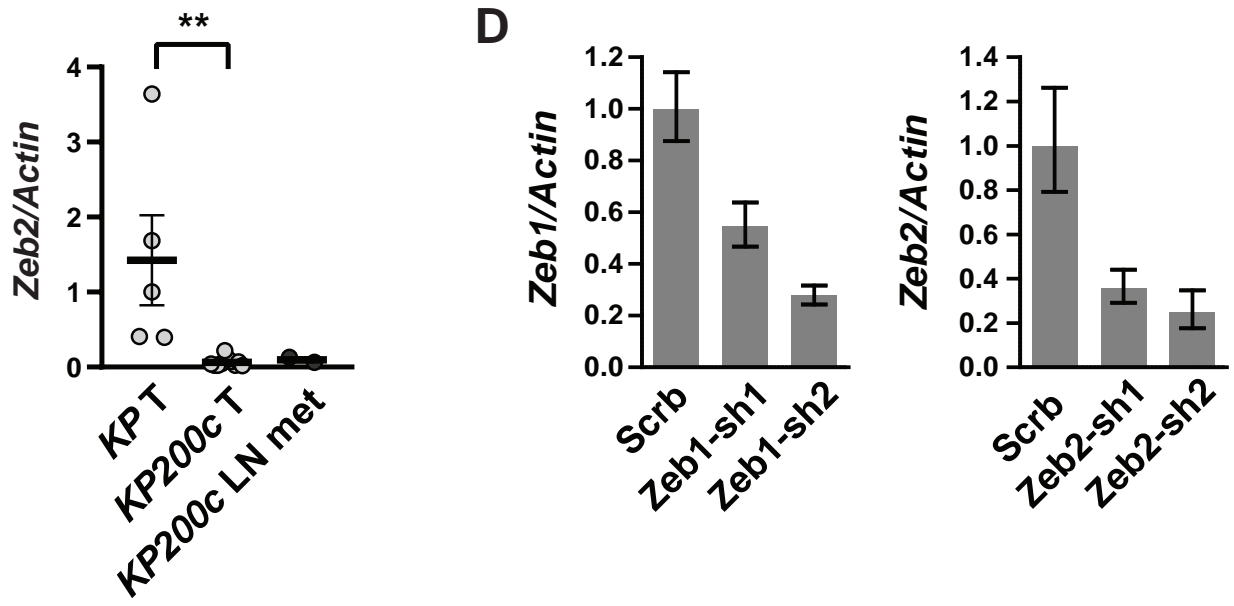


\section{Supplementary Figure S4 cont'd}
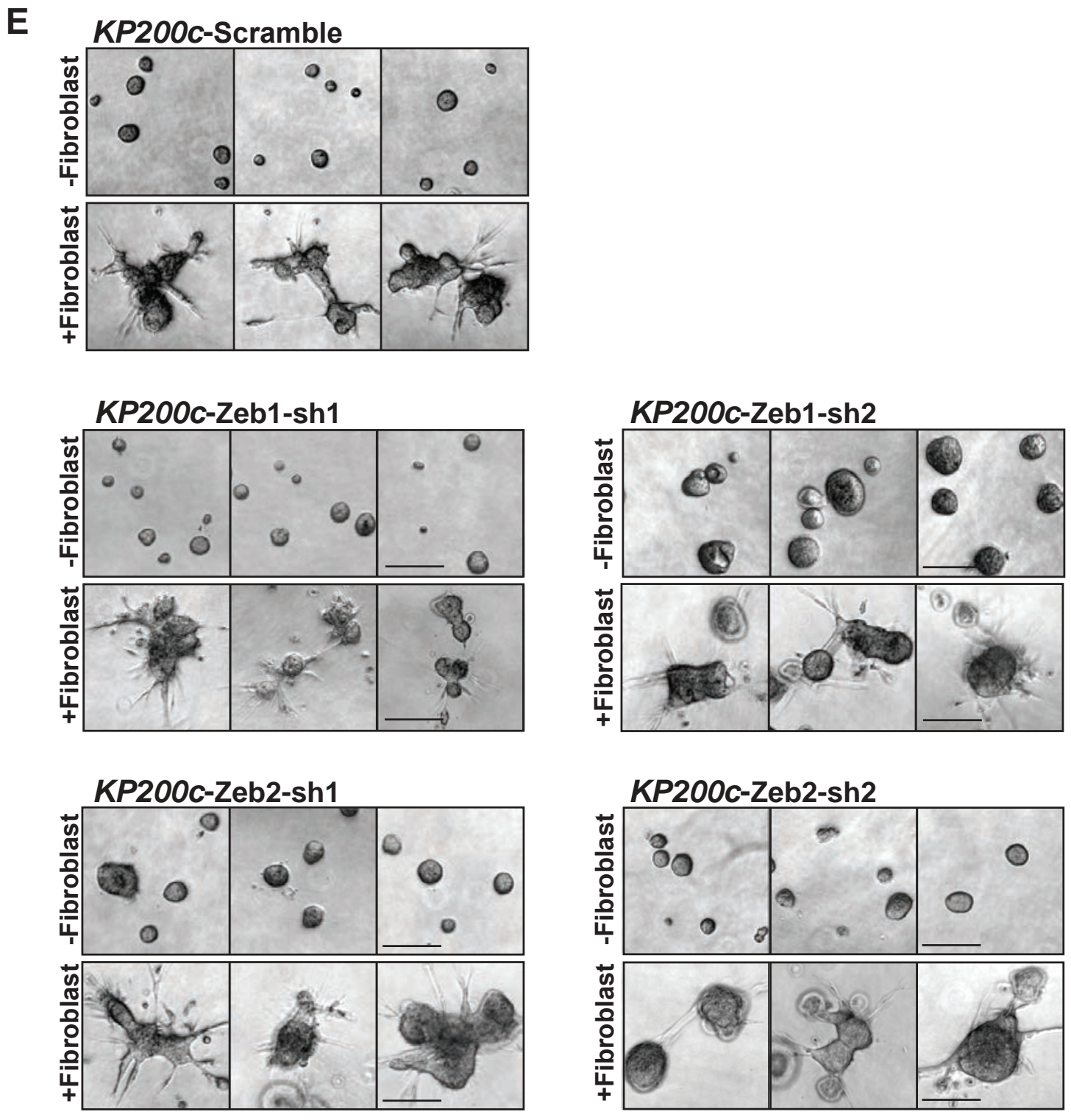

G

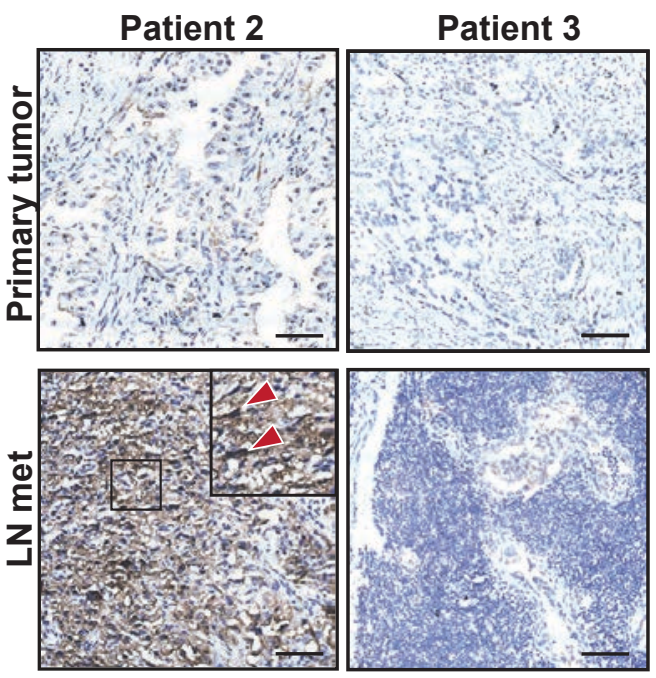

Patient 4
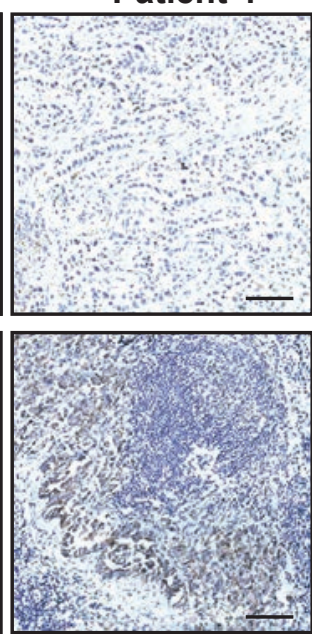

Patient 5
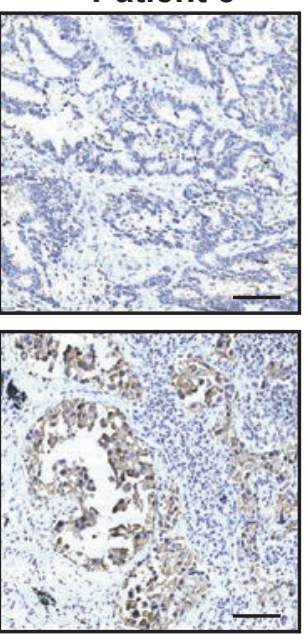

Patient 6
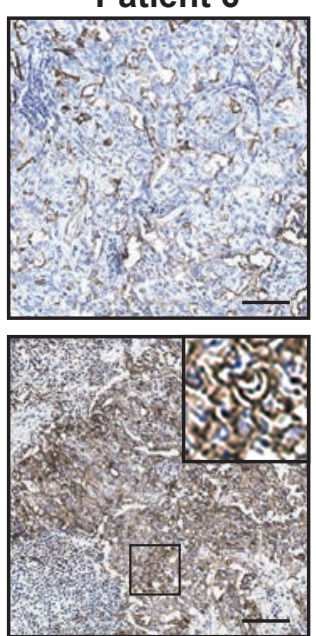

Patient 7

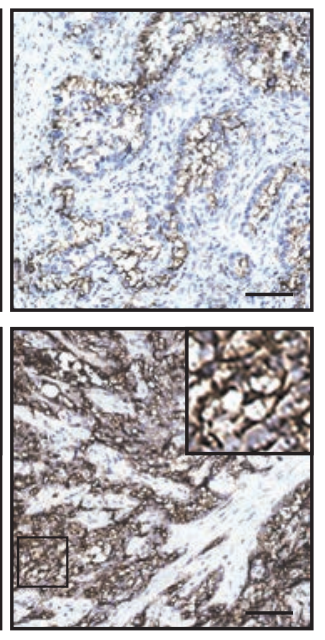

NICD 